\title{
Abstracts and authors of the 8th International Conference on Spatial Cognition: Cognition and Action in a Plurality of Spaces (ICSC 2021)
}

\author{
1. Abstracts of Keynotes \\ 2. Abstracts of Symposia \\ 3. Abstracts of Talks \\ 4. Abstracts of Posters \\ 5. Author Index
}

\section{Abstracts of Keynotes}

\section{KEYNOTE 1:}

\section{Merging familiar and new senses to perceive and act} in space

\section{Marko Nardini}

\section{Durham University, England}

Our experience of the world seems to unfold seamlessly in a unitary $3 \mathrm{D}$ space. For this to be possible, the brain has to merge many disparate cognitive representations and sensory inputs. How does it do so? I discuss work on two key combination problems: coordinating multiple frames of reference (e.g., egocentric and allocentric), and coordinating multiple sensory signals (e.g., visual and proprioceptive). I focus on two populations whose spatial processing we can observe at a crucial stage of being configured and optimised: children, whose spatial abilities are still developing significantly, and naïve adults learning new spatial skills, such as sensing distance using auditory cues. The work uses a model-based approach to compare participants' behaviour with the predictions of alternative information processing models. This lets us see when and how-during development, and with experience-the perceptual-cognitive computations underpinning our experiences in space change. I discuss progress on understanding the limits of effective spatial computation for perception and action, and how lessons from the developing spatial cognitive system can inform approaches to augmenting human abilities with new sensory signals provided by technology.

\section{KEYNOTE 2:}

\section{Making Spaces: The Natural, Cultural, Cognitive} and Social Niches of Human Activity

\section{Barry Smith}

\section{University of Buffalo, United States}

A niche is, roughly speaking, an environment in which an organism or group of organisms can live and act. Drawing on the ecological psychology of J. J. Gibson and Roger Barker, and especially on
Barker's theory of behaviour settings, I will explore different kinds of niches for human activity, and then show how they become variously combined together in reflection of different kinds of human activity. One important category of combined niches are the spatial contexts which serve as niches for different kinds of conversational activityfor instance, in a queue, in an art gallery, on a railway platform, at a party. I will use examples of such combined niches in order to illustrate some of the reasons why machine emulations of human performance in conversation are faring so poorly when compared to some of the more impressive recent achievements in the field of artificial intelligence.

\section{KEYNOTE 3:}

\section{Spatial Thinking, Cognitive Mapping, and Spatial} Awareness

Toru Ishikawa

INIAD Toyo University, Tokyo, Japan

Spatial ability has attracted scientific and pedagogical interest particularly recently because it plays important roles in academic learning and everyday activities. Because of the importance, thinking about space has now been extensively discussed in a broader context of spatial thinking. A type of spatial thinking that is of particular significance to people's daily lives is cognitive mapping, a series of psychological processes of acquiring, representing, and using knowledge about spatial environments. The skill of cognitive mapping shows large individual differences; however, the task of spatial orientation and navigation poses great difficulty for some people (especially people with a poor sense of direction). With the existence of such large individual differences, researchers' attention has shifted from asking whether people's cognitive maps are maplike to investigating how and why people differ so much in the fundamental ability of cognitive mapping. In this talk, I will look at why spatial is special for human cognition and behavior and overview the characteristics of cognitive-behavioral approaches to the study of spatial cognition. I particularly discuss the motivation and findings in the research on cognitive mapping from the perspective of individual differences, and consider the possibility of improving cognitive mapping by training. I also discuss long-term cognitive consequences 
of the use of satellite navigation on people's spatial awareness and possible ways to adjust social design to the wide variations in spatial aptitudes and preferences among people.

\section{KEYNOTE 4:}

\section{Skill and the perception of space}

\section{Anthony Chemero}

\section{University of Cincinnati, United States}

In this talk, I will contrast the roughly Kantian conception of spatial cognition that currently dominates the cognitive sciences with a more thoroughly embodied approach. This latter view of spatial cognition, derived from phenomenological philosophy and ecological psychology, makes our abilities for skilled bodily action the key determinants of our spatial cognition. I will argued that the latter approach is a more fruitful way to approach certain of our abilities to perceive and think about space. I will finish with some examples showing how this embodied understanding of spatial cognition translates to work in the laboratory. (This draws on my collaboration with Stephan Käufer.)

\section{KEYNOTE 5:}

\section{How environmental movement constraints shape the neural code for space}

\section{Kate Jeffery}

\section{University College London, United Kingdom}

The neural code for space in mammals, including humans, is centred on the hippocampal place cells, which are active in particular places in the environment. A longstanding question is: how do place cells "know" where to be active? Research in animals over several decades has revealed the existence of numerous other spatial cell types which supply the place cells with information: examples include the grid cells in entorhinal cortex and the head direction cells, found in numerous brain areas. These neurons use a mixture of information about the animal's movements and information about the environment to create and update their map-like representation. This talk will review these findings and then describe recent work from my and other labs suggesting that the map of space is not fixed, but rather is adapted to the movement constraints of the environment. For example, neural coding is different on a horizontal versus vertical plane, and different in a lattice than on a surface. These findings are pointing towards a more flexible spatial code in which the map is adapted to the environment rather than being rigidly anchored to the world. This may explain why some types of navigational behaviour in humans look sub-optimal under some conditions.

\section{KEYNOTE 6:}

\section{Brains in Space-Effects of Extreme Environments on Brain Plasticity and Spatial Cognition}

\section{Alexander Stahn \\ Center for Space Medicine and Extreme Environments, Germany}

Physical and social environments are key to physiological and behavioral plasticity across species. Extreme environmental conditions can dampen this response, and even have a detrimental effect. Empirical evidence from animal studies shows that social isolation, immobilization, and altered gravity can have profound effects on brain plasticity, and particularly brain areas associated with spatial cognition. Whether these effects translate to humans is not well understood. In this talk, I will argue that spaceflight research in humans provides unique opportunities to gain new insights into the role of experiential diversity on brain and spatial cognition. I will show how acute exposure to varying gravity levels, spaceflight, longduration bed rest, social isolation, and Antarctic expeditions can affect brain plasticity and spatial abilities, and how we can utilize these settings to develop new approaches to mitigate potential adverse neurobehavioral effects associated with such extreme conditions.

\section{KEYNOTE 7:}

\section{Bodily self-consciousness and egocentric space}

\section{Olaf Blanke}

\section{Swiss Federal Institute of Technology (EPFL), Lausanne, Switzerland}

Based on latest evidence on the relevance of interoceptive bodily signals (cardiac and respiratory signals) and multisensory exteroceptive bodily signals for self-consciousness (bodily selfconsciousness), I will propose an integrated neural system reconciling these two largely separated views. I argue that such an integrated system is based on torso-centered signals in a distributed cortical network and delineate how it accounts for fundamental aspects of bodily self-consciousness, focusing in particular on conscious selflocation. Presenting examples how self-location impacts the construction of personal and peripersonal space as well as spatial navigation behavior, I conclude by highlighting the relevance of these findings for egocentric space.

\section{KEYNOTE 8:}

\section{The neuroaesthetics of architectural spaces}

\section{Anjan Chatterjee}

\section{University of Pennsylvania, United States}

People in developed countries spend over $90 \%$ of their time in built environments. Yet, we know little about its pervasive and often hidden effects on our mental state and our brain. Despite growing interest in the neuroscience of architecture, much of this scholarship has been descriptive. The typical strategy is to map knowledge of the brain onto constructs important to architecture. For a programmatic line of research, how might we transform descriptive neuroarchitecture into an experimental science? I will describe our efforts to do exactly that. We hypothesized aesthetic responses to architectural interiors reduce to key psychological dimensions and that these psychological dimensions evoke specific neural markers. Using Psychometric Network Analysis (PNA) and Principal Components Analysis (PCA) and responses to curated images, we identified three components: coherence (ease of organizing and comprehending a scene), fascination (informational richness and generated interest), and hominess (personal ease and comfort). Coherence and fascination are well-established dimensions for natural scenes. Hominess was a new dimension related to architectural interiors. Central to all three communities in the PNA was emotional valence. We also reanalyzed data from an earlier fMRI study in which participants made beauty and approach-avoidance decisions while viewing the same images. Regardless of task, the degree of fascination covaried with neural activity in the right lingual gyrus. In contrast, coherence covaried with neural activity in the left inferior occipital gyrus only when 
participants judged beauty, and hominess covaried with neural activity in the left cuneus only when they made approach-avoidance decisions. The visual brain harbors hidden sensitivities to architectural interiors that are captured by the dimensions of coherence, fascination, and hominess. I will conclude by describing challenges for experimental neuroarchitecture and how we might move forward.

\section{KEYNOTE 9:}

From embodying tool to embodying alien limb: sensorymotor modulation of personal and extrapersonal space

\section{Anna Berti}

\section{University of Turin, Italy}

Years ago, it was demonstrated (e.g. Rizzolatti et al. 2000) that the brain does not encode the space around us in a homogeneous way, but through neural circuits that map the space relative to the distance that objects of interest have from the body. In monkeys, relatively discrete neural systems, characterised by neurons with specific neurophysiological responses, seem to be dedicated either to represent the space that can be reached by the hand (near/peripersonal space) or to the distant space (far/extrapersonal space). It was also shown that the encoding of spaces has dynamic aspects because they can be remapped by the use of tools that trigger different actions (e.g. Iriki et al. 1998). In this latter case the effect of the tool depends on the modulation of personal space, that is the space of our body. In my talk I will review and discuss selected research, which demonstrated that also in humans; 1 spaces are encoded in a dynamic way; 2 encoding can be modulated by the use of tool that the system comes to consider as parts of the own body; 3 body representations are not fixed, but they are fragile and subject to change to the point that we can incorporate not only the tools necessary for action, but even limbs belonging to others people.

\section{Abstracts of Symposia}

\section{SYMPOSIUM 1: General abstract}

\section{Behaviors and collective spatial representations in interactive situations}

\section{Convenors: Bernard Guelton ${ }^{1} \&$ Teriitutea Quesnot ${ }^{2}$ \\ ${ }^{1}$ University of Paris 1 Pantheon-Sorbonne, France \\ ${ }^{2}$ Université de Bretagne Occidentale, France}

Background: The exploration of urban areas has been transformed since the arrival of instrumental mapping tools like Google Maps. At the same time, the growth Web 2.0 and the democratization of smart mobile devices have facilitated the design of shared cartographic media (e. g. OpenStreetMap, Waze, etc.). Beyond utilitarian matters (i.e., getting from A to B), a significant number of contemporary artistic practices have taken up these tools to explore new collective and (re)creative uses. Collective exploration became a performative medium that allows the hybridization of spatial experiences in common or remote environments. While previous studies attempted to identify the impact of navigation tools on our cognitive representations, none really addressed the impact of collective interactive tools between urban explorers. In this context, to what extent are instrumental and shared maps likely to change our behaviors and spatial representations? Aim: This symposium aimed at comparing behaviors and collective spatial representations in interactive situations in urban areas. The questions of the way as tools of remote interactions between participants impact their individual and collective representation of space will be the main objectives. Approach: The symposium will examine different ways and tools of interactions between participants in urban space and the mutual impacts of collective behaviors on collective and individual representations of space. Questions of the analytical means of these impacts will be investigated.

\section{SYMPOSIUM 1: Submission 75}

\section{Analytical means of spatial representation resulting from collective interactions}

\section{Bernard Guelton}

\section{University of Paris 1 Pantheon-Sorbonne, France}

Background: In addition to numerous navigation applications in urban environments, specialized applications now allow to interact collectively in a playful or functional perspective. The means of evaluating these behaviours and the collective representations involved are now a major challenge. Aims: The aims are therefore to find out whether or not significant differences can be identified between subjects with or without a device in terms of behaviours and the individual and collective representations involved, and to evaluate the methods used to identify these differences. Methods: The first step is to focus on static analyses (drawings and graphs) in order to confront three groups of subjects with: (1) passive trackings, (2) individual cartographic application, and (3) collective and interactive cartographic application, in a situation of individual and collective interactions through the exploration of an urban district northern suburbs of Paris called: "Plaine St Denis", $1.50 \mathrm{~km}^{2}$. The cartographic tools utilised is a specific application designed by the company ORBE. In addition to a statistical analysis of the landmarks, the creation of graphs from the drawings made is a second method used. Results: In the first results obtained, the way of spatially locating oneself differs according to whether or not the subjects use collective interaction tools. On the other hand, the confrontation of the graphs constructed from the drawings does not reveal sufficiently significant differences. Conclusion: These first results should soon be able to be re-evaluated through a dynamic approach that confronts the trackings made with the dynamics of the drawings recorded in video.

\section{SYMPOSIUM 1: Submission 90}

\section{Construction of a geographic database for analysis of collective mental maps}

\section{Mattia Bunel}

\section{Université de Bretagne Occidentale, France}

Background: In addition to numerous navigation applications in urban environments, more specialized applications now allow to interact collectively in a playful or functional perspective. The means of evaluating these behaviors and the collective representations involved are now a major issue and challenge. Aims: The aim of this research is to digitise and analyse the location information represented on mental maps by subjects of an experiment made in the ANR CORES. Methods: The construction of the location information database is based on the manual identification of landmarks on mental maps, and then relating them to the objects in OpenStreetMap. This database allows us to analyse the types of objects used by the subjects in their 
mental map, and the differences between the Euclidean space and the subject's mental space representation. Results: The first results are a database built from the mental maps drawn by 120 subjects and a static analysis of the types of objects represented. This analysis makes it possible to highlight the most frequently used types and instances of objects, and the differences between the different groups of subjects. Conclusion: This initial analysis makes it possible to highlight the differences in representations that may exist between the different mental maps used by the subjects to represent the space they have visited. Our short-term objective is to compare these results with the GPS traces of the subjects during their journey, to see whether there is a link between the journey and its representation.

\section{SYMPOSIUM 1: Submission 118}

\section{Panorama and vista of street contribute perceptual salience in collective maps}

\section{Sudhir Kumar Pasala \\ Andhra University College of Engineering (Autonomous), Visakhapatnam, India}

Background: Most of the literature on landmark salience describes the physical aspects of a particular structure that acts as focal point of visual attention during navigation in urban landscapes. It could be true that these structures attract the navigator in reaching the place, and meanwhile, there is growing research on other navigational strategies. We propose that panorama and vista as a cursor of continuity in visual attention could contribute better experiential navigation in urban streets. Aims: To find what references or landmarks are chosen by individuals for mental representation of a neighbourhood. Methods: A neighbourhood of varied landscape in Paris-suburbs is assessed to understand navigational strategies of graduate students in relation to their day-to-day needs. Ten such graphs drawn by the participants locating the landmarks and references were analyzed. We shortlisted the landmarks and such references that were widely chosen by the participants to assess their salience from OpenStreetMap geographic database. Results: Apart from the start and end point which are the same, the total number of reference points chosen by the participants is around 1000 in number. However, from the data, there are 10 frequently chosen references and landmarks that were analyzed from OpenStreetMap geographic database which further could be correlated with gaze information from eye-tracking. Characteristics of panorama and vista for the streets of Quai-François Mitterrand and Rue de Saint-Gobain CIFAFashion respectively have plausible contribution to perceptual salience. Conclusion: Panorama and vista contributes visual continuity of a place by gathering more information enhancing cognitive salience of collective maps.

\section{SYMPOSIUM 1: Submission 179}

\section{Impact of social interactions on the acquisition of spatial knowledge}

\section{Teriitutea Quesnot}

\section{Université de Bretagne Occidentale, France}

Background: The rise of online mapping, mobile Internet, and smart devices allows to renew the content analysis of externalized mental maps in the form of sketch maps. Thanks to these technologies, it is now possible to connect physically distant people who move within the same space at the same time. Aims: Our research aimed at evaluating the impact of interactions produced via a shared mapping application on the acquisition of spatial knowledge and its restitution in the form of sketch maps. Methods: Two groups walked freely in the Plaine Saint-Denis district of Paris. Each movement was recorded in real time using a tracking device. Once the exploration finished, the participants were asked to draw on a sheet of paper the environment they had just walked through.

- Group 1: 40 people who walked individually without any tools.

- Group 2: 8 groups of 5 people interacted with each other (commenting and posting photos about places) using a shared mapping mobile application on which the real-time paths of each walker appeared on a white background.

Preliminary results and Conclusion: Preliminary analyses of tracking data and sketch maps show that unlike participants of Group 1, individuals of Group 2 mentioned on their mental maps landmarks that they did not visit during their exploration. In other words, those results suggest that collective interactions seem to have an impact on the process of spatial knowledge acquisition.

\section{SYMPOSIUM 2: General abstract}

\section{Navigation and environment representation: personality and individual differences}

\section{Convenors: Chiara Meneghetti \& Francesca Pazzaglia}

\section{General Psychology Department, University of Padova, Italy}

Navigation is an essential everyday activity. Research has shown that individuals vary in their ability to perform navigation tasks (such as retracing the same path or finding a new one), and tasks that involve managing environment representations (such as judging directions or map drawing). One line of research in spatial cognition is investigating the factors influencing different individuals' recall performance after learning an environment. Among several such factors, individual differences in spatial thinking can have a relevant role. These differences range from visuospatial cognitive abilities (which are objectively measurable) to inclinations (self-reported environment-related preferences). Inclinations include our preferences regarding how we move around (such as pleasure in exploring), the conviction that we will be successful in wayfinding tasks (spatial self-efficacy), and a growth mindset when it comes to environment learning, which are representative of individual motivation and environment-related emotional aspects. Other aspects of individual differences in navigation and environment-learning accuracy include factors such as gender (males vs. females), and age (children vs. adults vs. the elderly). This symposium aimed to present fresh evidence of numerous individual factors capable of optimizing the accuracy of our environmental representations. It will show that navigation accuracy relates to visuospatial cognitive abilities (such as mental rotation) as well as personal attitudes and inclinations regarding the environment. Individual differences are interrelated too. It is worth comparing females and males on their navigation skills, for instance, to see how to improve the former's performance (females generally navigate less well than males) by sustaining their emotional-motivational beliefs (or self-efficacy) regarding spatial tasks, or by preventing the activation of a gender stereotype. Overall, this symposium offers a key to our better understanding of these issues and new insight on how to examine navigation and spatial learning accuracy in relation to personal abilities and dispositions, better contextualizing the differences from one individual to another. 


\section{SYMPOSIUM 2: Submission 28}

\section{The impact of stereotypes and self-assessment on navigation performance}

\section{Ineke van der Ham}

Department of Health, Medical, and Neuropsychology, Leiden University, Netherlands

Background: Gender stereotypes with regard to spatial functioning are quite prevalent, a male favouring stereotype for spatial tasks like reading a map appears to be common. In addition, we have recently found a systematic pattern of overestimation in males and underestimation of females when it comes to navigation ability. Aims: The aim of the current work is to study the impact of gender stereotypes and biases in self-assessment on navigation performance. Methods: In one questionnaire $(\mathrm{N}=985)$ and two experiments $(\mathrm{N}=120 ; \mathrm{N}=173)$, the characteristics of gender stereotyping for spatial navigation are addressed, and it is assessed how stereotype beliefs impact selfassessment and objective performance. Results: Findings indicate that a male favoring stereotype for navigation is strong and is supported more by male and young adult participants, in comparison with female and older adults. Stereotypes were strongest for male participants with relatively high levels of male gender identification. A direct relation of stereotype belief to objective performance appears limited. When manipulating stereotype belief with false scientific evidence, we found that although females show emotional responses and males rational responses to the manipulation, the impact on objective performance is limited and depends on the content of the spatial task used. Conclusion: Although gender stereotypes for spatial navigation are strong, especially for younger males, the impact on cognitive performance is limited, depending on task characteristics. Moreover, males and females have different emotional and rational responses to stereotyped information. Further implications of these findings to assessing spatial functioning in males and females will be discussed.

\section{SYMPOSIUM 2: Submission 32}

\section{Spatial learning and gender: cognitive, motivational, and social factors}

\section{Laura Miola, Chiara Meneghetti, Veronica Muffato, Enrico Toffalini, Francesca Pazzaglia}

Department of General Psychology, University of Padova, Italy

Background: The literature has documented gender differences in spatial learning and spatial navigation, showing an advantage for men. However, a systematic understanding of why women perform worse than men in spatial tasks is still lacking. To date, studies have focused mainly on cognitive variables (e.g. visuo-spatial ability, VSA). Aims: Our research project aimed to investigate whether motivational, affective and social factors (e.g. gender stereotype) might play a role in the observed gender differences. Methods: In two studies, structural equation models were used. In Study 1, participants completed questionnaires on general spatial self-efficacy (SE), sense of direction, growth mindset, gender spatial stereotype and an Implicit Association Test (SA-IAT). In Study 2, participants learned a route in a virtual environment, and afterwards they indicated the starting point and located landmarks on a map. Before performing each task, participants reported their self-efficacy referred to the task (specific SE). General SE and VSA were assessed using, respectively, a questionnaire and two tests (Jigsaw Puzzle and Mental Rotation). Results: We explored whether sense of direction and spatial self-assessments can be influenced by growth mindset and gender stereotypes. We also analyzed the role of VSA and SE in mediating the relation between gender and spatial learning accuracy. Results indicated that SE and VSA fully mediate the association between gender and spatial learning performance. Conclusion: Our findings highlight the contribution of SE in gender differences in spatial learning and point to the importance of also considering non-cognitive factors in explaining gender differences in spatial dominion.

\section{SYMPOSIUM 2: Submission 101}

\section{Does GPS use Impair Navigation Skills? Insights from a Cohort Analysis}

\author{
Chuanxiuyue He, John Protzko, Alexis Kunz \\ Jonathan Schooler, Mary Hegarty
}

\section{University of California, Santa Barbara, United States}

Background: With the development and prevalence of Global Positioning Systems (GPS), there is a growing concern that we may be losing our navigation abilities by relying too much on GPS technologies. Over-reliance on GPS may also be related to increased spatial anxiety. One way to investigate these issues is via cohort effects. Aims: In this study, we examine navigation ability and spatial anxiety of those who learned to drive before and after GPS technologies were widely used by the public. Methods: We surveyed 963 adults (533 female, 430 male) across the adult age range (18-70) on measures of GPS use, spatial anxiety, and self-reported sense of direction. Results: Results indicated that GPS use and spatial anxiety decrease with age while self-reported sense of direction increases with age. Moreover, for participants under 35 (who had GPS available in their early driving years), GPS use was positively correlated with spatial anxiety (.45) and negatively correlated with sense of direction (.29). However, these correlations were significantly weaker (.36 and .06 respectively) for participants in the 35-70 age range. Conclusion: These results are consistent with the concern that younger adults may be negatively impacted in developing their navigation abilities, and consequently experience more spatial anxiety due to reliance on GPS.

\section{SYMPOSIUM 2: Submission 176}

\section{Individual Differences in Spatial Memory for a Large-Scale Virtual Environment}

Morgan A. Saxon ${ }^{2}$, Phillip Fernberg ${ }^{1}$, Sarah H. CreemRegehr $^{2}$, Jeanine K. Stefanucci ${ }^{2}$, Brent Chamberlain ${ }^{1}$

\section{${ }^{1}$ Department of Landscape Architecture, Utah State University, United States \\ ${ }^{2}$ Department of Psychology, University of Utah, United States}

Background: Prior work shows there are individual differences in how we represent, explore, and navigate space. While much research is dedicated to recall for routes and landmarks, less is known about how we create meaningful categories of and within a space, such as how we delineate residential areas from commercial areas. Such skills are consequential for situations in which categorizing a space influences behavior such as decisions about the safety of traversing a specific region of a city. Aims: The current study aimed to evaluate individual differences in spatial measures after navigating in a virtual environment. Methods: Participants navigated a desktop or largescreen virtual environment modeled after a suburban cityscape 
populated with various landmarks. Participants either navigated a fixed route or a route that presented choices between continuing forward and turning onto a different street. We measured wayfinding strategies, spatial abilities, and spatial anxiety and tested participants' recall for route, landmarks, and scenes. We used a novel task to test whether and how participants categorized sections of the environment into districts (i.e., residential, commercial, park, industrial). Results: Preliminary findings show differences in the accuracy of drawing the proportions of different districts and suggest gender may relate to these differences. No differences in route memory were found based on route manipulation or spatial ability. Conclusion: Meaningful categorizations of spaces are a useful measure of spatial memory for large-scale environments and an individual differences approach may help us understand these types of memories.

\section{SYMPOSIUM 3: General abstract}

\section{Cognition and Action in Virtual Spaces}

\section{Convenors: Gabriella Antonucci \& Marco Iosa}

\section{Sapienza University of Rome, Italy; IRCCS Fondazione} Santa Lucia, Italy

The current pandemic and the following lockdown have forced people and companies to get creative about how to interact and communicate with other people, leading certain connective technologies to become essential aspects of our lives. In a period in which the physical environment around us has been restricted to a room or house, VR development allows to enhance the space in which we can interact with other people. Some innovative research fields on spatial cognition are involving the use of VR to create fully-controlled artificial environments in which the subject has the sensation to be embedded, and virtual avatars give to the subject new bodies for interacting with virtual objects and in the virtual space. We will treat the perceptions and the behavioural aspects related to the use of a virtual body in a virtual space. In particular, Tieri will talk about the illusions elicited by VR, such as the embodiment over a virtual body and its effect on the space perception, whereas Committeri will focus her seminar on the space cognition related to peripersonal and extrapersonal space in the virtual environment. Then, the use of VR in neuroscience and neurorehabilitation will be faced. VR in this field can be used for the treatment of patients, as in the study of Iosa, to teach rehabilitation students, as in the study of Roduta Roberts, or to implement training and diagnosis tools as in the study presented by Maselli. In the study by Roduta Roberts, a virtual environment was developed for simulating a clinical room with the student and a virtual client trained using an artificial intelligence to provide realistic responses upon questioning simulation on student performance to manage stress/ anxiety within an occupational therapy program. The study of Maselli and collaborators presents a virtual task in which participants had to intercept virtual balls thrown from avatars animated with the full body kinematics previously recorded in a real throwing task. Besides providing a tool for training complex motor and spatial skills, the study was designed to assess the participants' ability in extracting task relevant information from the observation of others' full-body movements, which is known to be a key skill for successful social interaction that may be impaired in some neurological conditions. Finally, in the study of Iosa and colleagues, a VR-task was developed for allowing subjects, by moving their hand on a virtual canvas, to have the illusion of painting an art masterpiece (such as Michelangelo's Creation of Adam or the Birth of Venus of Botticelli), finding an increment of motor performance and a reduction of perceived physical load in comparison with a control task in which the canvas was simply colored.

\section{SYMPOSIUM 3: Submission 198}

\section{The Michelangelo effect: art modifies spatial trajectories on virtual canvases}

\author{
Marco Iosa ${ }^{1,2}$, Merve Aydin ${ }^{1}$, Carolina Candelise ${ }^{1}$, \\ Giovanni Morone ${ }^{2}$, Stefano Paolucci ${ }^{2}$, \\ Gabriella Antonucci, \\ ${ }^{1}$ Sapienza University of Rome, Italy \\ ${ }^{2}$ IRCCS Fondazione Santa Lucia, Italy
}

Background: The vision of an art masterpiece is associated with brain arousal by neural processes occurring quite spontaneously in the viewer, even eliciting a response in the motor areas of the observers imagining to perform the represented actions or to explore the scene. In the neurorehabilitation of patients with stroke, art observation and creative art therapy have been used for reducing psychological disorders and enhancing physical and cognitive functions. Aims: We developed an art-therapy protocol based on virtual reality to favor the neurorehabilitation of patients with stroke. Methods: Twenty healthy subjects (experiment 1) and four patients with stroke (experiment 2) performed a virtual reality task in which they had the illusion of painting some art masterpieces by moving their paretic hand on a virtual canvas (or just to color the canvas in control condition). Their performances were evaluated in terms of kinematics measured by the virtual system and by the self-report assessments using USEQ-scale and NASA Task Load Index. Results: The level of usability resulted high. Moreover, despite the motor task was the same for art and control conditions, the artistic one was performed by healthy subjects with shorter trajectories on the space of the canvas $(p=0.001)$ and with a lower perception of physical demand $(p=0.049)$. In experiment 2 , only the patients treated with artistic stimuli showed a reduction in the erroneous movements out of the canvas space $(p<0.05)$. Conclusion: We found an improvement in the performance when interacting with an artistic stimulus, and we called it as Michelangelo effect.

\section{SYMPOSIUM 3: Submission 203}

\section{The Impact of Virtual Space in a Simulation to Enhance Student Performance}

Mary Roduta Roberts ${ }^{1}$, Nathanial Maeda ${ }^{2}$, Shaniff Esmail ${ }^{1}$, Pegah Firouzeh ${ }^{2}$, Maliheh Hadizadeh ${ }^{2}$, Merve Aydin', Iris Chao ${ }^{1}$,Emma Chrenek ${ }^{1}$

${ }^{1}$ Department of Occupational Therapy, University of Alberta, Canada

${ }^{2}$ Faculty of Rehabilitation Medicine, University of Alberta, Canada

Background: Virtual reality (VR) can be effective technology to promote excellent learning skills in different fields of study including medical procedure development. Indeed, researchers focus on VR design and virtual space since well-constructed simulations produce positive outcomes. Aims: The aim of this presentation was to describe the development of the virtual space within a VR simulation and its impact on participation, student performance, and stress/anxiety within an occupational therapy program. Methods: The virtual space replicated a clinic room with an examiner and patient. The participant did not assume the body of an avatar, only hands. A mixed-method study design was used. Twenty-eight students completed the VR simulation and self-report measures of mental workload, usability, presence, and affect. Students later completed a performance-based examination comprising a simulated client interview. Eight students 
participated in two focus groups. Results: Demographics from census data $(\mathrm{N}=121)$, of which the participants belong, indicated an average age of 24.3 years with $\mathrm{N}=10(8 \%)$ identifying as male. Self-report measures indicated students' experienced decreased presence and high mental workload and did not always achieve their intended goal within the virtual space. Qualitative findings support the students' reports of stress during the VR simulation, however, some stress was attributed to external factors. Regarding performance, examination scores between students who participated in the VR simulation and those who did not were significantly different. Conclusion: Findings suggest revisions to the virtual space are required to enhance immersion and improve usability as a necessary prerequisite to support skill development and reduction of stress.

\section{SYMPOSIUM 3: Submission 205}

\section{Macro-affordance: extrapersonal distance and locomotion in virtual environments}

\section{Annalisa Tosoni, Emanuele Cosimo Altomare, Giorgia Committeri}

\section{University G. d'Annunzio of Chieti-Pescara, Italy}

Background: The Gibsonian notion of affordance and the actionspecific perception account have been massively employed to characterize the mutual interdependence between cognitive-perceptual processes and body state/action possibilities within the surrounding environment. This relationship has been mainly explored from the perspective of action within the hand-related reachable/peripersonal space (micro-affordance) or using explicit judgments about the extrapersonal space (EP). Aims: Testing the existence and the underlying neural correlates of an affordance relationship between the spatial features of the EP (i.e. distance) and locomotion, as privileged action to cover distance and access information contained within the EP. Methods: We used a realistic 3D virtual environment presented on a wide-field screen and an incidental priming paradigm (go/no-go) in which participants executed a footstep ahead (vs. simple release) in response to pictures containing tridimensional objects located at far versus near EP distance or framing the environment from far versus near distance. Transcranial magnetic stimulation (TMS) was then applied on a parietal region related to planning and execution of footrelated actions (vs. sham and control region). Results: We observed a walking-related facilitation effect for far versus near EP distance and a significant reduction of the effect following TMS over the foot-related region versus control stimulation. Conclusion: The $3 \mathrm{D}$ virtual environment and large visual-field stimulation allowed to unveil an advantage for locomotion actions towards far EP. Based on the parallelism with the micro-affordance effect, this facilitation was referred to as "macro-affordance" and was proposed to reflect an automatic activation of mechanisms for spatial navigation and exploration.

\section{SYMPOSIUM 3: Submission 207}

\section{Catching Virtual Throws: assessing predictive skills in sensorimotor interactions}

\footnotetext{
Antonella Maselli ${ }^{1,2}$, Paolo De Pasquale ${ }^{1,3}$, Paolo Tommasino ${ }^{1}$,Francesco Lacquaniti ${ }^{1,4}$, Andrea d'Avella ${ }^{1,3}$

${ }^{1}$ Laboratory of Neuromotor Physiology, Santa Lucia Foundation, Rome, Italy

${ }^{2}$ Institute of Cognitive Sciences and Technologies, CNR, Rome, Italy
}

${ }^{3}$ Department of Biomedical and Dental Sciences and Morphofunctional Imaging, University of Messina, Italy ${ }^{4}$ Department of Systems Medicine and Center of Space Biomedicine, University of Rome Tor Vergata, Rome, Italy

Acting in a dynamic environment requires predictive abilities to infer others' intentions or the outcome of observed actions. Accurate motor responses should be planned in advance to compensate for latencies in sensory processing, motor planning and execution. Recent research has shown how social skills in humans are rooted in the ability to formulate predictions based on the observed movements, while impairments of such ability are associated with conditions such as autism and neurodegenerative diseases. We present an immersive virtual reality (IVR) study aimed at assessing the predictive mechanisms associated with action observation in complex motor tasks. Participants had to intercept balls thrown from virtual characters animated with full-body kinematics recorded from real throwers. The experiment included three conditions, with either only the ball trajectory visible, or only the throwing kinematics visible, or both. Predictive skills were assessed according to the recorded interception kinematics, by comparing the three visibility conditions. Results showed that vision of the throwing kinematics, in addition to ball trajectory, significantly improves interceptive performance. In addition, participants, who were not experts in throwing sports, were able to predict to some extent the direction of the thrown ball based on the throwing kinematics alone. High hits rates for visible balls indirectly tested for depth perception and the use of internal models of gravity in (IVR). The possibility to assess predictive skills in neurotypical subjects support our setup both as a valid platform for training interactive motor skills, and as a potential tool for the early assessment of neurological conditions.

\section{SYMPOSIUM 3: Submission 208}

\section{Virtual bodies in virtual spaces}

\section{Gaetano Tieri}

Virtual Reality Lab, University of Rome Unitelma Sapienza, Italy; IRCCS Santa Lucia Foundation, Rome, Italy

Background: When we observe a virtual body from a first-person perspective through a virtual reality (VR) headset, a condition in which our body is substituted by the virtual one, we typically have the illusory sensations that the virtual body belongs to us (Feeling of Ownership) and that we are responsible for its actions (Agency). These aspects represent two main components of the embodiment and are fundamental for bodily self-consciousness and for interacting optimally with objects and other individuals. Aims: Evidence highlights that the embodiment is a plastic construct that relies on brain regions involved in multisensory integration but its effect on the space perception is still scarce. In this talk, I will present a series of our recent VR studies where we investigated, at behavioural and physiological level, the effect of virtual embodiment on the perception of peripersonal space, i.e. the space surrounding our body. Methods: I will illustrate a novel methodological VR approach to create a conflict between the vision (of the virtual limbs in space) and the proprioception (of the real hidden limb) in order to investigate how and whether the peripersonal space is modulated. Results and Conclusion: Results suggest that the embodiment of virtual body affects the perception of our limbs in space and that the boundaries of the peripersonal space seem to enlarge for including the virtual limbs. Finally, I will discuss about the possible application of this evidence for future motor learning and rehabilitation approaches. 


\section{SYMPOSIUM 4: General abstract}

\section{From individual to social spaces: low- and high-level mechanisms of interpersonal interactions}

Convenors: Chiara Fini ${ }^{1}$ \& Vanessa Era ${ }^{2,3}$

${ }^{1}$ Department of Dynamic and Clinical Psychology, Sapienza University of Rome, Italy

${ }^{2}$ SCNLab Department of Psychology, Sapienza University of Rome, Italy

${ }^{3}$ IRCCS, Fondazione Santa Lucia, Rome, Italy

Every day we orient our actions towards objects and other individuals, flexibly adapting spatial and psychological distances. In such continuous "adaptive calibration" we satisfy different needs ranging from, e.g., grasping a can to quench the thirst, caressing the loved ones, or guessing the best friend's thoughts to increase the reciprocity, to finally, forming a space of intersubjectivity where the other is a cobuilder of shared meanings. The sense of touch allows us to physically interact with the world: it determines the boundaries of the body and, in conjunction with other sensorial channels, shapes body representations. It is not just our sensorial bridge with the world, but it participates in action orientation and coding by anticipating specific object features and action components (micro-affordances). Touch is also the sense through which we are connected with another human being. Indeed, the very first interaction between a new-born baby and its mother happens through the skin. Such physical connection facilitates the emergence of different kinds of intimate relations that we experience across life. However, the social stimuli surrounding us are more than those detected by the skin. Humans are equipped with other "distal" senses (e.g., vision, hear) and a sophisticated mental system to interpret other people's intentions. The ability to impute mental states, such as beliefs, desires, or intentions, to oneself and to others is known as Theory of Mind (ToM). We do not need explicit reasoning to infer other people's mental states. We process them also without being aware of them and when other people's perspectives are immaterial. The spontaneous inference of other people's mental state contributes to advance the idea that our mind is structured to be in a constant implicit dialogue with other human beings. In keeping with the Vygotskian perspective, the self emerges within social relations, as a space of intersubjectivity necessary to structure and cultivate it. This symposium will focus on recent studies elucidating how interacting with the physical environment (including objects and other individuals) shapes physical and psychological representations of an "interindividual space". More specifically, the first and second sessions will focus on how the physical space around an individual is shaped by interactions (mediated by the sense of touch) with objects and individuals (low-level mechanisms of interactions). While the first one will elucidate how the sense of touch serves the function of action coding and orientation, the second one will deal with the social function of the sense of touch in intimate relations. The last two sessions, instead, will discuss how interpersonal interactions create shared psychological representations (high-level mechanisms of interactions). In the third session, neuroscientific evidence on the implicit ToM, as the natural ability to interpret other people's thoughts, will be presented. In the fourth session, a new theoretical framework in which the self is considered as emerging within social relations will be proposed and the implications for psychiatric, pedagogical and ethics will be considered.

\section{SYMPOSIUM 4: Submission 66}

\section{Haptics, lateralized affordances and object interactions during the pandemic}

\section{Arthur-Henri Michalland}

Université Montpellier-LIFAM, France; Ballab-Body Action Language Laboratory, Rome, Italy

Recent works showed that the role of haptic signals (tactile and kinesthetic) in action selection may have been underestimated (Thébault et al. 2018,2020). Thus considering haptic properties of objects and actions may deepen our understanding of affordances (Gibson 1979) in the peripersonal space: specific action components that are potentiated when seeing an object, depending on the contextual cues and the task at hand (Tucker \& Ellis 1998; Borghi 2018). We assessed whether (a) biases for visually (right hand) or haptically (left hand) guided movements (Stone \& Gonzales 2015), and (b) the links between touch and behavioral immune system (Schaller \& Park 2011) could play a role in action coding and potentiation. We studied response times with prickly and soft objects while wearing gloves or not (Study 1), the force exerted with objects with various weights and softness (Study 2), and the response times with objects that may be a source of fomite infection through other's touch (study 3 , ongoing). In studies 1 and 2, with right hand responses participants took into account more features of an experimental situation than with left hand (glove wearing, object position, prickly feature), but movements were less flexible (no force modulation) which prevented to consider specific haptic features (weight and softness). In study 3 , we expect the touch from potentially infected people to inhibit affordance activation. In conclusion, haptic properties are part of the contextual features that modulate affordances, and other signals derived from haptic properties may also modulate affordance activation, providing insights on action control and selection.

\section{SYMPOSIUM 4: Submission 124}

\section{From Theory of Mind to social interaction: the case of implicit processes.}

\section{Lara Bardi}

\section{Institut des Sciences Cognitives-CNRS, France}

Background: Theory of Mind (ToM) refers to our ability to impute mental states to oneself and to others. This ability has long been thought to require complex inference. A mounting body of data, however, suggests that others' perspectives may affect our behavior in an implicit/automatic way. Aims: We analyzed the behavioral and neural mechanisms of implicit ToM and asked whether implicit and explicit forms of mentalizing are, at least partially, overlapping. Methods: We looked at the BOLD signal for false belief processing and directly compared implicit and explicit ToM task versions. In both versions, participants watched videos of a scene including an agent who acquires a true or false belief about the location of an object. At the end of the movies, participants had to react to the presence of the object. In a separate experiment, noninvasive brain stimulation was used to interfere with brain activity during task performance. Results: Behavioral performance in object detection was influenced by the belief of the agent, irrespective of task instructions. The activation of the temporo-parietal junction (TPJ) and the anterior medial prefrontal cortex showed the same pattern for implicit and explicit ToM. TMS of the TPJ confirmed the causal role of this area in implicit ToM. Conclusion: Results support the idea that adults implicitly track others' beliefs, without the need of explicit 
reasoning, even when perspectives of others are irrelevant for the task at hand. Our fMRI and brain stimulation results suggest that explicit and implicit forms of ToM share at least some neural mechanisms

\section{SYMPOSIUM 4: Submission 147}

\section{The influence of sexual orientation on nonverbal interactions}

\author{
Martina Fusaro ${ }^{1,2}$, Matteo P. Lisi ${ }^{1,2}$ \\ ${ }^{1}$ Social Neuroscience Laboratory, Fondazione Santa Lucia, \\ Rome, Italy
}

\section{${ }^{2}$ Sapienza, Università degli Studi di Roma \& CLNS@Sapienza, Istituto Italiano di Tecnologia, Rome, Italy}

Background: Immersive virtual reality (IVR) offers a way to recreate sensory environments that can duplicate reality and elicit veridical experiences. Aims: Here using IVR we investigated the behaviour of men and women dealing with virtual characters to measure the quality of different types of nonverbal interactions (i.e., touch and interpersonal distance). Methods/Results Studyl: In the first study, heterosexual, gay and lesbian participants observed their virtual body while receiving touches on different parts (including taboo zones) from a male or a female avatar. Results showed that touches on different areas elicited lifelike sensations that were dependent upon the biological sex and the sexual orientation of the participants. Moreover, skin conductance responses showed that touches on the intimate zones (breast and genitalia) elicited the highest reactivity compared to other zones. Methods/Results Study2: In the second study, heterosexual and non-heterosexual men and women were asked to provide comfort-distance judgements in relation to approaching male or female virtual avatars. Results showed that same-sex comfortdistance was smaller for non-heterosexual compared to heterosexual participants. Moreover, the larger distance toward the male avatar kept by heterosexual men was also associated with the implicit prejudice (measured with an implicit association test) toward gay men. Conclusion: The results from our studies suggest that sexual orientation, along with the most investigated role of sex, plays a fundamental influence in regulating touch and interpersonal distance. Importantly, IVR seems to represent a unique opportunity to explore the psychophysiology of sexuality in the regulation of nonverbal interactions.

\section{SYMPOSIUM 4: Submission 159}

\section{'I interact therefore I am'-Human becoming in and through social interaction}

\section{Dimitris Bolis \\ MPI of Psychiatry, Germany}

Drawing inspiration from real-life experience through a Vygotskian lens, in this talk, we attempt to motivate a systematic shift of focus from being to becoming; in fact becoming-with. More concretely, leaning on the dialectical method, cultural-historical theory, and recent developments of social computational neuroscience, we argue that a multiscale analysis of social interaction might allow us to scientifically reconsider the self beyond the individual, where it really emerges, unfolds, and manifests itself-in social relations. In this light, we discuss the dialectical misattunement hypothesis, which views autism and broadly psychopathology as a dynamic interpersonal mismatch, rather than a (disordered) function of single brains.
We then operationalize these hypotheses on the basis of a novel empirical framework, namely two-person psychophysiology, which measures and analyzes the multiscale dynamics of social interaction. Deploying this framework, we empirically demonstrate that real-time dynamics of social interaction do shape behavior and cognition in both collective and individual dimensions-even beyond awareness-lending support to second-person and enactivist proposals. With regard to psychopathology, we show that it is primarily the mismatch of autistic traits-not traits per se-which predicts core aspects of interpersonal attunement in real-life social relationships. Taken together, we try to break free from dichotomies such as internalism/externalism or healthy/patient, in theoretical, methodological and empirical regards. In a nutshell, our approach to human becoming in and through social interaction encourages a social change pertinent to various fields of human research and practice, ranging from psychiatry and pedagogy to ethics and artificial intelligence.

\section{SYMPOSIUM 5: General abstract}

\section{Conceptualizing musical features through spatial cognition}

\section{Convenor: José L. Besada}

\section{Complutense University of Madrid, Spain}

Music is a human universal, although each culture has developed musical practices in particular ways. Music mainly involves sound and time. We are often able to spatially identify the source of a sound, but many of its most fundamental features are not concrete. This lack of concreteness is also a consubstantial feature of time. However, the use of spatial attributes for conceptualizing time and further sound features, although different across cultures, are ubiquitous in human societies. In the case of Western culture, the historical development of a device for musical notation has fixed two main image schemas: pitches are ordered within a vertical distribution, and time flows from left to right. The second one is inherited from the Western writing system, the former evolved from the neume notation in the Middle Ages. Trained musicians are obviously accustomed to these schemata, but also naïve people understand many of their related expressions by means of an implicit acquisition of musical concepts. For instance, Western individuals do not need to read music for somehow understanding and identifying what "high" or "low" pitches are. The above notwithstanding, the human easiness for acquiring spatial conceptualizations of musical features does not mean that is a simple, straightforward, static, and immovable task. Quite the contrary, it is a complex, intricate, and dynamical process that admits, especially from the side of trained musicians, highly sophisticated manipulations with goal-oriented creative purposes. This symposium is aimed at shedding some light about the way in which different populations (non-trained, students, and professional musicians) cognitively deal with spatial conceptualizations of disparate musical features, namely (1) pitch recognition, (2) conceptualizations of musical time, (3) the definition, perception, and modelling of harmonic distances, (4) the 2-D geometrization of harmonic sequences, and (5) correspondences between visual imagery and listening processes. All these approaches are different but converge in pointing to pitch and time as the main spatialized features of music in Western traditions. The symposium purposely gathers scholars with multidisciplinary profiles-encompassing musicology, music theory, music education, cognitive science, biology, physics, mathematics, and computer science-for a fruitful transversal discussion. 


\section{SYMPOSIUM 5: Submission 48}

\section{Confronting composers' visual imagery with listeners' experience of music}

\author{
José L. Besadal , Marc Vidal Badía ${ }^{2}$ \\ ${ }^{1}$ Complutense University of Madrid, Spain \\ ${ }^{2}$ Ghent University, Belgium
}

Background: Contemporary compositional practices are grounded on Western standard musical notation, which incorporates defined image schemata for organizing time and pitch. This spatial context for developing musical creativity has fueled the value of composers' visual imagery for conceiving their techniques. Conversely, the impact of these sorts of cross-modal correspondences on the audience remains quite more unexplored. Aims: The goal of this paper was to understand and measure the influence of visualizing and understanding an image representing a deformed space on the aural perception of several music excerpts that were inspired by this same image. Methods: We have taken four musical excerpts of Alberto Posadas' Anamorfosis for large ensemble, in which the composer tried to transfer the homonymous painting technique into music. Each excerpt concerns an "anamorphic" manipulation of pitches, time, timbre, or all mixed variables. A cohort of university students were asked to make a schematic draw, under certain geometrical restrictions, for depicting the visual image each musical excerpt evoked them. The students were divided into two groups: the control one just performed the tasks, and the other group was previously informed about the painting that inspired Posadas and his note program providing an explanation of what an anamorphosis is. Results and Conclusion: We can currently provide some qualitative information about tendencies in drawings for each excerpt/musical feature as well as some coincidences with their respective sonograms. We have however started to explore statistical tools for image analysis in order to manage more accurate quantitative data (spectrum, density, patterned structures)

\section{SYMPOSIUM 5: Submission 60}

\section{Contemporary composers twisting timelines: A cognitive approach}

\author{
Cristóbal Pagán Cánovas ${ }^{1,2}$, José L. Besada ${ }^{3}$ \\ ${ }^{1}$ Murcia University, Spain \\ ${ }^{2}$ University of Tübingen, Germany \\ ${ }^{3}$ Complutense University of Madrid, Spain
}

Background: How musicians think about time is crucial across their practices. Just like many other activities, music involves spatial notions to conceptualize time with the support of material or virtual devices. Musicians spatialize time in sophisticated ways to serve their purposes in composition and performance. Aims: To provide a sample of compositional practices in which contemporary music composers have curved straight timelines in order to manage their musical ideas. This practice provides insights into the general use of spatial structures to build complex temporal ideas. Methods: We have scrutinized a large sample of compositional sketches by Iannis Xenakis, Pierre Boulez, Gérard Grisey, and Kaija Saariaho. We have confronted these sketches with the theoretical writings they have left, and we have analyzed the impact of these sketches on their factual musical outcomes. Results: We have found many deformed timelines and unusual anchoring templates within the production of these authors: from spiral or springy timelines, to seismograph- or gearwheel-like templates. Although they may seem a priori "exotic," all them are goal- oriented. A simply straight timeline would not have been useful for the same artistic purposes. When considered within the theoretical framework of blending theory, our case studies provide insights into the manipulation of temporal concepts through spatial cognition. Conclusion: The study of sketches from compositional practices from the perspective of spatial cognition and conceptual integration is a largely unexplored territory, which can provide a multimodal view of the use of spatial properties for creativity across verbal, graphical, and musical representations.

\section{SYMPOSIUM 5: Submission 91}

Modeling perceived distance among music collections: A machine-learning approach

\section{Erica Bisesi}

University of Montreal, Canada; GATM-Gruppo Analisi e Teoria Musicale, Italy

In a previous study, we compared five contrasting models of distance between collections in post-tonal music (pieces by Webern, Bartok, Scriabin), and tested predictions against perceptual data (Bisesi 2017, 2020). We considered an adaptation of the Parncutt (1988) psychoacoustical model of distance between chord-roots, three models of distance based on interval similarity (Rahn 1979-1980; Morris 1980; Lewin 1987), and atonal voice leading (Straus 2003). Relevant sets were extracted by means of analyses of segmentation, internal symmetries, and pitch-class set theory. Profiles of distance between sets' pairs were computed by means of algorithms (Ariza 2002; Bisesi et al. 2019). We asked 20 trained musicians to evaluate the perceived distance in 32 pairs of sets on a rating scale (randomly presented in three different realizations: arpeggios, arpeggios in ascending order, chords) and correlated empirical data with predictions by different models. In an exploratory fitting approach, we found preference for a combination of models based on psychoacoustics and interval proximity, with exclusion of the voice leading $(\mathrm{rWebern}=0.66$; $\mathrm{rBartok}=$ $0.84 ;$ rScriabin $=0.96 ;$ rAll $=0.82$ ). In this study, we are extending previous research by: (1) including the Tonnetz as a groundwork for measuring the distance (Cohn 1997); (2) applying different machine learning techniques to data analysis (e.g., multiple regression, neural networks); (3) interpreting final results in terms of the cognitive strategies which oriented participants' evaluations, as explored by means of a questionnaire; (4) comparing and integrating the assumptions different models of distance are based on (i.e., perceptual, algebraic, and geometric).

\section{SYMPOSIUM 5: Submission 93}

\section{Spatial mental anchorpoints during identification of notes' pitch by musicians}

\section{Pierre Legrain ${ }^{1}$, Alain Letailleur ${ }^{1}$, Erica Bisesi ${ }^{2}$ \\ ${ }^{1}$ Institut Pasteur, France \\ ${ }^{2}$ University of Montreal, Canada}

Background: Much of the research in cognitive sciences aimed at defining the brain activities associated with a given cognitive task. The consensus is that these activities should be similar in all healthy individuals. However, the idea of individual variability in cognitive mental strategies has emerged recently. Aims: We sought for individual diversity in a simple auditory task: the recognition of notes' pitch. Methods: Through a systemic approach based on introspection, we explored the mental strategies used by musicians. Sixty-seven 
participants, both music students and professionals, were interviewed. We developed a mixed approach combining qualitative analyses of interviews with quantitative statistical methods. Results: Our study identified various mental strategies involving intermediate stages of selection or activation of cognitive bricks which contribute to the correct identification. We called them "mental anchorpoints." We demonstrated that such mental anchorpoints have different natureauditory, visual and kinesthetic, and can act both separately and in combination. We have recently performed additional experiments to objectify these results. Pitch identification is perturbed by different means (auditory or visual). As expected, visual mental representations are used by some musicians to identify pitches, but not by others. Therefore, the choice of mental anchorpoints for pitch recognition is a matter of individual specificities. Conclusion: Based on these findings and for this particular case study, we hereby propose an interpretation of how the mental process of spatialization is connected to the mechanisms structuring music perception.

\section{SYMPOSIUM 5: Submission 99}

\section{Conceptualizing chord relationships via spatial visualization within the Tonnetz}

\author{
Moreno Andreatta ${ }^{1,4}$, Erica Bisesi ${ }^{2,3}$, \\ Corentin Guichaoua ${ }^{4,1}$, José L. Besada ${ }^{5}$ \\ ${ }^{1}$ Institute for Advanced Mathematical Research, CNRS- \\ University of Strasbourg, France \\ ${ }^{2}$ Music Faculty, University of Montreal, Canada \\ ${ }^{3}$ Institut Pasteur, France and CNRS, Paris, France \\ ${ }^{4}$ Music Representations Team, IRCAM, Paris, France \\ ${ }^{5}$ Department of Musicology, Complutense University of Madrid, \\ Spain
}

The Tonnetz is a conceptual lattice diagram depicting the tonal space, suitable for visual representations of harmonic relationships. Despite a fair number of studies modelling perceived distance among pitch collections (e.g., Krumhansl 1998; Milne and Holland 2016; Bisesi 2017), very few is known about the cognitive strategies adopted by individuals to apprehend the abstract structures which underlie such geometrical representations of music theory. Open questions concern whether the structures implicitly encoded by the Tonnet $z$ have some perceptual foundation, somehow innate in human cognition, and how to grasp and consequently teach the mathematical principles on which it rests. To answer these questions, we adapted a web application of the Tonnetz for developing an empirical experience. Thirty participants with very different cognitive backgrounds ( 15 scientists and 15 trained musicians) hear 12 parsimonious chord sequences, in the double format of sounds and coloured patterns displayed on the graphical interface, and are asked to recognize the visual position of the last chord of each sequence among six proposed solutions. The task is presented twice: first, being unaware of what the Tonnetz is; second, after a short video tutorial. The mental strategies brought into play when elucidating the answer are grasped by means of a followon questionnaire. We are currently analyzing the responses, in order to elucidate to what extent previous musical or mathematical knowledge facilitate the task, the influence of the video tutorial, and the different ways of integrating visual and auditory modalities.

\section{SYMPOSIUM 6: General abstract}

The body as the link between mind and space: a philosophical and neuroscientific discourse to understand the role of architecture in cognitive and emotional processing

\author{
Convenor: Giovanni Vecchiato ${ }^{1}$ \\ ${ }^{1}$ Institute of Neuroscience, National Research Council of \\ Italy, Parma, Italy
}

Architecture influences actions and emotions being the main stage of our everyday social interactions. This symposium will guide the auditory through selected neuroscientific knowledge and philosophy scholars to explain how architectural features impact on low and high level brain mechanisms, ultimately shaping human cognition. Recent research on how we experience architecture has highlighted that built spaces affect us much deeper at bodily and mental level thus shaping our actions and emotions (Mallgrave 2015). These talks will lead to the understanding that architectural experience goes beyond the mere visual processing and that sensorimotor mechanisms are fundamental to shape perception of space thus defining the whole social environment around us. The discourse will start from the perspective of the visual neuroscience showing how figure-ground perception (Papale et al. 2018), shape coding (Papale et al. 2020) and architectural expertise (Olivito et al. in preparation) are all features which could potentially inform architects to move from a "focused" design approach, i.e. considering architecture at the center of the visual scene, to a more "defocused" way of thinking about their projects, i.e. taking into account how we perceive at the periphery of the visual scene (Wallis et al. 2019). However, since architecture is a multisensorial subject, the mere visual processing does not explain the whole spatial perception which is completed through body representations. The phenomenology of Edmund Husserl and MerleauPonty and the pragmatism of John Dewey show that embodied experience is a communal nexus of meaningful situations, expressive gestures and practical actions. From this point of view, architectural space is formed by human situations long before it is structured geometrically (Vesely 2004). The space beyond our skin becomes the field of possibility - tempered and conditioned by the possibilities it afford for action (Böhme 2017). In configuring the concrete situations of daily life, architecture serves as the corporeal and topological ground of human becoming (Robinson 2021; Robinson and Pallasmaa 2015). Therefore, today it is urgent to integrate all the acquired knowledge to provide a neurophysiological and computational model of architectural affordances reflecting human experience (Djebbara et al. 2019). From this perspective, active inference and enactivism can be exploited because they centralize action-perception as a unifiedprocess reflected in sensorimotor dynamics for the inference of the world (Friston 2010; Jelić et al. 2016; O'Regan and Noë 2001). Essentially, the dynamics are transition-patterns that accentuate the action in the genesis of perceptual experience, revealing that architecture enters the loop of cognition by designing actions. To date, the outcome is an attempt to go beyond traditional architectural methods and to synthesize phenomenological arguments with prominent neuroscientific theories. Among such theories, embodied cognition sets the motor system as the core hub processing spatial features, personal and other's actions and emotions (Rizzolatti and Sinigaglia 2016). Recent findings already showed evidence of embodied mechanisms in architectural perception (Vecchiato et al. 2015). Recognizing also that observers are more accurate in recognizing body expressions when these are emotionally congruent with the emotional valence of the environment (Kret and de Gelder 2010), it becomes relevant to understand the role that sensorimotor processes play in cognitive adaptation mechanisms generated by architecture. We will show that 
such adaptation deeply impacts in social interactions shaping our daily behavior and mental states (Presti et al. 2020). This symposium fosters the design of environments towards the creation of proper atmosphere in specific places, and it is addressed to scientists, scholars, as well as private and public administration to highlight the impact that living environments have on society.

\section{SYMPOSIUM 6: Submission 6}

\section{Neurophenomenology for Architecture: an embodied and enactive inference approach}

\section{Zakaria Djebbara}

Department of Architecture, Design, Media and Technology, Aalborg University, Denmark

The dynamic coupling within the brain, body, and environment has recently gained traction, making neuroscience attractive to architects. The aim was to draw parallels between the nature of human experience and computational neuroscience to guide future studies. As our perception is enacted by the sensory and motor system, each action changes the perceived environment in line with our expectations. Our expectations are bound by our afforded actions, shaped by architectural affordances. Since affordances depend on the fit between the body and capacities for movement, our understanding of architecture relies on sensorimotor processes. Computational neuroscience proposes an auspicious Bayesian framework of cognition that provides a meaningful explanation of neuronal activity by way of 'active inference'. Both active inference and enactivism centralize actionperception as a unified process reflected in sensorimotor dynamics for accessing the world. This demonstrates how the environment emerges in the dynamics as a loop rather than as an end-product. Essentially, the dynamics are transition patterns that accentuate the action in the genesis of experience, revealing that architecture enters the loop of cognition by designing actions. Integrating sensorimotor activity with active inference yields a computational model of architectural affordances that in turn reflects human experience. The outcome is an attempt to go beyond traditional architectural methods by synthesizing phenomenological arguments with a prominent theory of brain activity. To this end, a neurophenomenological account of the emergence of architectural experience is developed through an enactive inference, which in turn suggests how architecture impacts experience.

\section{SYMPOSIUM 6: Submission 17}

\section{A walk in the visual neuroscience: feedbacks and lessons learned}

\section{Paolo Papale}

\section{Netherlands Institute for Neuroscience, Netherlands}

Do we really need to understand the brain to design better buildings? In this talk, I will present a personal perspective from an architect undertaking visual neuroscience research. I will not offer a structured view on the relationship between neuroscientific knowledge and architectural theory, but I will rather share the few lessons learned while randomly walking in such a different field. I will mention the results from two experiments on figure-ground perception that could potentially inform architects and help them moving from a "focused" design approach, i.e., considering architecture at the center of the visual scene, to a more "defocused" way of thinking about their projects, i.e. taking into account how we perceive at the periphery of the visual scene. Also, I will talk about what we know on shape coding and what properties are actually relevant to our perception of forms. In conclusion, I will talk about the critical role of expertise in shaping perception and confidence: what does it mean to be an expert? I will show some preliminary data on how expertise can shape our perception and could lead to a biased approach to design.

\section{SYMPOSIUM 6: Submission 18}

\section{Linking architecture and emotions by the recognition of virtual body expressions}

\author{
Paolo Presti ${ }^{1,2}$, Davide Ruzzon ${ }^{3,4}$, Pietro Avanzini ${ }^{1}$, \\ Fausto Caruanal, Giovanni Vecchiato ${ }^{1}$
}

${ }^{1}$ Institute of Neuroscience, National Research Council of Italy, Parma, Italy

${ }^{2}$ Department of Medicine and Surgery, University of Parma, Italy

${ }^{3}$ University IUAV, Venezia, Italy

${ }^{4}$ TUNED, Lombardini22 s.p.a., Milan, Italy

Background: Architecture deeply impacts on our life being the stage of our everyday social interactions. Aims: The aim of this pilot study was to unveil the relationship between the architectural experience and the perception of body expressions as evidence of their common neural underpinnings. Here we present an adaptation effect paradigm developed in virtual reality to show how the experience of virtual architectures affects the following judgment of emotional body postures. Methods: Two virtual architectures (adapting stimuli) were designed modifying height of the ceiling, width of the side walls and height of the windows to induce low and high level of perceived arousal. A database of emotional body kinematics was exploited to build virtual postures (target stimuli) with avatars representing low, middle and high level of arousal. These adapting and target stimuli were presented in sequence to 10 subjects using a head-mounted display. Following this stimulation, subjects were asked to make a binary judgement about the avatar's bodily arousal. Results: The percentage of low responses across all body postures was significantly higher when subjects experienced the high arousing architecture. This result shows that, depending on the emotional characterization of the virtual environment, subjects differently perceived the affective state represented by the avatar's body posture. Conclusion: This pilot paves the way to the investigation of specific architectural feature contributing to the adaptation effect. Showing that architecture interacts with our ability to comprehend the others' affective state, a human-centered design would be beneficial for promoting healthy social interactions.

\section{SYMPOSIUM 6: Submission 19}

\section{Architecture as Situated Poetics}

\section{Sarah Robinson}

\section{Aalborg University, Denmark}

Despite the contributions of the Cognitive Sciences toward an understanding of embodiment, architectural theory and practice remains entrenched in the Enlightenment interpretation of the body as mechanism-known through its external coordinates operating in neutral space. In place of this obsolete notion, this paper/talk presents the body as a body-in-action (Böhme 2017). This understanding of the body is the basis for an approach called "Situated Poetics," which draws on the phenomenology of Edmund Husserl and Merleau-Ponty 
and the pragmatism of John Dewey, who together showed that embodied experience is a communal nexus of meaningful situations, expressive gestures, habitual movements and practical actions. In this view, space is not neutral but a field of bodily and intentional possibility formed by human situations long before it is structured geometrically (Vesely 2004). This approach argues that in configuring the concrete, practical and habitual situations of daily life, architecture serves as the corporeal and topological ground of human becoming. (Robinson and Pallasmaa 2015 and Robinson 2021).

\section{SYMPOSIUM 7: General abstract}

\section{Cognitive maps for abstract information: From working memory to long-term memory}

\author{
Convenors: Wim Fias ${ }^{l}$ \& Jean-Philippe van Dijck ${ }^{2}$ \\ ${ }^{1}$ Department of Experimental Psychology, Ghent Univer- \\ sity, Belgium \\ ${ }^{2}$ Department of Applied Psychology, Thomas More \\ University \\ College, Belgium
}

The efficiency of knowledge representations strongly depends on how well information is mentally structured and organized. Space is often considered as a means to achieve an efficient organization. The idea of knowledge being spatially organized goes back to Tolman and Honzik (1930) who proposed that such organization is realized by creating cognitive maps. Because of the discovery of place and grid cells in the hippocampus and entorhinal cortex, this idea has recently revived and resulted in a neurocognitive framework of how cognitive maps are implemented in the brain. Currently, cognitive maps are on the foreground of research in both the domain of working memory and long-term memory. Interestingly, evidence in line with cognitive maps is not restricted to information that is explicitly spatial in nature (like in spatial navigation) but also extends to the representation of abstract information, ranging from verbal information in working memory to conceptual knowledge in long term memory. The present symposium brings together four talks that give an overview of recent progress in our understanding of how cognitive maps are created and used in both working memory and long-term memory, how they are implemented in the brain, how they are used in applied contexts, and how they can be recovered from natural language. Together, this symposium introduces the audience to recent developments in research and theory on cognitive maps as efficient means of knowledge representation. In a first talk, Cristoforetti et al. focus on working memory. They present an overview of their work showing that (1) serial order in verbal working memory is spatially coded along the horizontal dimension; (2) visuospatial attention is used to explore verbal working memory; (3) neural mechanisms are recruited that overlap with those used for spatial navigation and memory. In a second talk, Bottini will extend from working to long term memory. He will introduce different reference frames for cognitive maps. Cognitive maps can be either world-centered or self-centered, each relying on different neural systems (hippocampal regions and parietal cortex, respectively). Bottini will explain how a hippocampal-parietal network can account for the organization of conceptual knowledge across multiple reference frames, and how this knowledge can be used in thinking and reasoning. In the third talk, Salvaggio will demonstrate how the framework of cognitive maps can be applied to the context of number processing and mathematical problem solving. For this, he will present a series of studies to illustrate how solving mathematical problems is accompanied by systematic horizontal eye movements and pupil light responses. Finally, by applying advanced analysis on the statistical distributions in natural language, Rinaldi explores the boundaries of the tight relationship between cognitive maps for conceptual knowledge and sensorimotor experience of space (which is explicitly or implicitly assumed in most cognitive map theories). These analyses show that statistical regularities available in language corpora are sufficient to reconstruct map-like information, both of geographical and more abstract information. This raises interesting questions regarding the nature and origin of cognitive maps.

\section{SYMPOSIUM 7: Submission 37}

\section{Visualizing Mathematics: Insights from eye-tracking studies}

\section{Samuel Salvaggio \\ Université catholique de Louvain, Belgium}

It has been suggested that number manipulation involves shifting attention along a left-to-right oriented continuum. In an attempt to further our comprehension of this mechanism, I will present a series of eye-tracking experiments combining free visual exploration with auditory numerical tasks. The first experiment required participants to compare two-digit numbers to 45 . A leftward ocular drift was observed while participants were listening to numbers smaller than the reference in comparison with larger numbers. The drift started early when the decade was sufficiently discriminating to allow a fast decision but later when the unit needed to be processed. The second experiment investigated the solving of addition and subtraction problems. A first difference was observed just after hearing the operator: the plus sign shifted the eye rightward compared to the minus sign. A second difference was observed later between problem offset and response onset: addition shifted the eye rightward compared to subtraction. In a last experiment, I measured the pupil light response of participants performing an auditory comparison task in front of a screen split into a dark and a bright area. Under strict control of central fixation, I showed that variations of pupil diameter reflected the side covertly attended by the participants as they classified numbers as smaller or larger than 45 . These data indicate that number manipulation involves covert attention shifts that surface at an oculomotor level and reflect the dynamic integration of the serial order of numbers, their organization in units and decades, and the polarity of arithmetic signs.

\section{SYMPOSIUM 7: Submission 39}

\section{Mapping serial order in working memory onto mental space}

\author{
Giulia Cristoforetti ${ }^{1}$, Jean-Philippe van Dijck ${ }^{1,2}$, \\ Wim Fias ${ }^{1}$
}

${ }^{1}$ Department of Experimental Psychology, Ghent University, Belgium

${ }^{2}$ Department of Applied Psychology, Thomas More University College, Belgium

The ability to maintain sequence of items (events, words, numbers) in working memory (WM) is a fundamental feature of our cognitive system. Previous research suggests that serial order WM is grounded in spatial attention. Verbal sequential information is spontaneously coded in mental space and retrieval of an item induces a covert spatial shift of attention depending on the position of the item in the sequence; begin elements are associated with the left side of space 
and end elements with the right. In the literature, there is a robust understanding of the cognitive mechanism involved; here we provide new evidence on the nature and the neural correlates of the involvement of spatial attention and spatial coding in serial order WM. In an EEG study, we observed that memory search across serially stored items involves spatial attention processes and generated the same electrophysiological signatures as those observed in the response to spatial cues in visuospatial WM and external space. Similarly, an eye-tracking study showed that eyes are spontaneously moving as a function of the ordinal position of a retrieved item belonging to a verbal WM sequence lacking spatial information. Our findings suggest that search in serial order WM is a dynamic process that exploits visuospatial attention. Ultimately, in an fMRI study with an MVPA approach, we observed that the intraparietal sulcus and the hippocampus contain information about the ordinal structure of the information memorized in a serial order WM sequence. Both regions are linked both to serial order coding and spatial coding.

\section{SYMPOSIUM 7: Submission 40}

\section{Spatial representations recovered from language}

\section{Luca Rinaldi}

Department of Brain and Behavioral Sciences, University of Pavia, Pavia Italy; Cognitive Psychology Unit, IRCCS Mondino Foundation, Pavia, Italy

Spatial representations are generally assumed to be fundamentally perceptual and necessarily grounded in sensory modalities. Here, I will try to challenge this assumption by taking advantage of distributional semantic models (DSMs), which represent word meanings as high-dimensional numerical vectors extracted from large amounts of natural language data. Despite these models lacking perceptual and spatial grounding as they rely on linguistic input only, I will first present evidence that DSMs are capable to reproduce not only the structural organization of maps (e.g., relative distances between cities) but also their directional arrangement (e.g., latitude and longitude coordinates). Perhaps more critically, I will demonstrate that linguistic statistical distributions can account for participants' performance in tasks requiring to estimate geographical position information, hence questioning the alleged need of spatial computations in the formation of such representations. I will then move on by discussing how language encodes the organizational structure of abstract information that is typically represented along space, with cardinal and ordinal proprieties for number and time that can be retrieved as well from text. I will finally probe whether the typical directional arrangement of the mental number line and the mental timeline can be derived from language. On these grounds, I will discuss the possible interplay between language statistics and culturespecific sensorimotor routines that may subserve the spatial representation of abstract information.

\section{SYMPOSIUM 7: Submission 82}

\section{Knowledge Across Reference Frames: Cognitive Maps and Image Spaces}

\section{Roberto Bottini}

\section{Center for Mind/Brain Sciences, University of Trento, Italy}

In human and non-human animals, conceptual knowledge is partially organized according to low-dimensional geometries that rely on brain structures and computations involved in spatial representations.
Recently, two separate lines of research have investigated cognitive maps, that are associated with the hippocampal formation and are similar to world-centered representations of the environment, and image spaces, that are associated with the parietal cortex and are similar to self-centered spatial relationships. I will review evidence supporting cognitive maps and image spaces and propose a hippocampal-parietal network that can account for the organization and retrieval of knowledge across multiple reference frames. Low-dimensional mental geometries such as cognitive maps and image spaces may play a crucial role in goal- directed cognition, analogical thinking, generalization, and the structuring of abstract conceptual domains.

\section{SYMPOSIUM 8: General abstract}

\section{Time Windows in Cognitive Processing}

\author{
Convenors: Yan Bao ${ }^{1}$ \& Ernst Pöppel ${ }^{1,2}$
}

${ }^{1}$ School of Psychological and Cognitive Sciences, Peking University, Beijing, China

${ }^{2}$ Institute of Medical Psychology, Ludwig-MaximiliansUniversität, Munich, Germany

It is a truism that temporal processing is essential for cognitive processing. The characteristics of temporal processing are, however, under debate. It is generally assumed that temporal processing is continuous, i.e. reflecting "Newtonian time" in classical physics. This concept, however, has to be questioned if one considers several features of sensory information processing. The transduction time on the receptor level is different between modalities being much shorter in audition compared to vision. If auditory and visual stimuli have to be related to each other when coming from common stimulus sources, the neural systems are confronted with a logistical problem as the central arrival time in the cognitive machinery is different. Furthermore, for distant objects in space the sound travel time is relevant, but not the speed of light. It has been observed that under optimal conditions at a distance of some 10 meter the sound travel time matches the retinal transduction time. Under ecological conditions the distance of objects is obviously not defined. What may be a way out that stimuli are temporally ill-defined? Empirical evidence suggests that "time windows" implemented pre-semantically may serve the purpose to overcome this logistical problem. Behavorial evidence suggests, using for instance temporal order thresholds as an indicator, that such time windows may have a duration of some tens on milliseconds. The contributions of Yifan Zeng and of Zhilin Zhang together with Xiaoxiong Lin deal with these problems. Zeng presents a new approach using neural oscillations as identified with EEG to determine thresholds for simultaneity; Zhang and Lin verify a holistic processing mode in measuring temporal order thresholds, whereas this high-frequency time window can be considered as a mechanism for complexity reduction of sensory information, a lower-frequency time window of a few seconds may serve a different purpose in cognitive processing. It has been observed in different domains of cognitive processing that there is a preference for segmenting information into temporal intervals of some three seconds. As in the case of the highfrequency time window, this lower-frequency time window is implemented on a presemantic level. This means that the time window is not implemented BY the information processed, but FOR the information to be processed. One experimental paradigm often used in studying this time window is temporal reproduction. In the contribution of Xuanyu Wang and Shunyu Shi the different neural components in this reproduction paradigm are experimentally disentangled. Another example for this time window comes from sensori-motor synchronization: Up to this temporal limit, the occurrence of stimuli can be anticipated which is not the case if the inter-stimulus interval is longer. 
In the study by Yuelin Liu, Taoxi Yang, and Yan Bao, it is shown that this limitation is overcome when visual stimuli in space have some spatial regularity. The 3 -second-time window is also related to aesthetic appreciations. In the study by Shuai Chen, Xinchi Yu and Ernst Pöppel bird songs are analyzed; under ecological conditions bird songs define an intra-species territory, but they show for humans also an aesthetic quality with a preference for the 3 -second time window. It has to be stressed that both the high-and the low-frequency time windows are not physical constants but temporal operating ranges.

\section{SYMPOSIUM 8: Submission 102}

\section{Holistic Processing in Temporal Order Perception of Tones}

\author{
Zhilin Zhang ${ }^{1}$, Xiaoxiong Lin $^{2,3}$ \\ ${ }^{1}$ Department of Psychology, New York University, United \\ States \\ ${ }^{2}$ Graduate School of Systemic Neurosciences, Ludwig- \\ Maximilian-University, Germany \\ ${ }^{3}$ Department of Neurosurgery, Klinikum rechts der Isar der \\ Technischen Universität München, Germany
}

Temporal order of two successively presented events in tens of milliseconds can be encoded holistically as a gestalt, such as apparent motion with spatially separate stimuli or pseudo glides composed of tones with different frequencies. This holistic mode can be regarded as an implicit temporal order processing alternative to analytical processing, where successive events are sequentially registered. In previous studies, it has been hypothesized that holistic and analytical processing modes might be responsible for the observed temporal order threshold difference between close and distant frequency tone pairs (Bao et al. 2013; Bao et al. 2014). Using a sensory adaptation paradigm, the present study aimed to demonstrate directly the holistic processing in temporal order perception of pure tones with different frequency distance. Participants were adapted to either upward or downward glides before they judged the temporal order of close or distant frequency tones. A varied time interval between the two successive tones was manipulated to measure the participants' performance. The results showed significant simultaneity criterion shift for both types of tones, indicating adaptation effect by glides. Furthermore, the magnitude of simultaneity criterion shift was larger for close frequency tones, where the participants had better discriminability of temporal order. The adaptation effect indicates that holistic processing is involved in temporal order perception of pure tones, which may match similar phenomena in spatial perception. Larger extent of holistic processing is associated with better temporal order discrimination as observed with close frequency tones, and the different magnitude requires further investigation with future research.

\section{SYMPOSIUM 8: Submission 112}

\section{A Potential Mechanism of Simultaneity Perception}

\section{Yifan Zeng}

Department of Psychology, Ludwig-Maximilians-Universität, Munich, Germany

High-frequency temporal perception has been demonstrated to be influenced by the phases of certain ongoing neural oscillations. However, previous studies suggest the corresponding frequency bands can vary substantially and be highly task-dependent. Moreover, the underlying mechanism of how ongoing oscillations provides a temporal reference frame for perceptual neural codes remains unclear. A general hypothetical theory is required. Here in this study, I propose that the cyclic shifts in neuronal excitability reflected by oscillations create critical phases only where the time reference exists. Stimuli would be represented by the corresponding time tag of the closest critical phase, leading to the threshold of simultaneity perception as half of the period of such oscillations. By presenting bi-stable visual stimuli pairs that can be perceived as either simultaneous or non-simultaneous, I examined the effect of pre-stimulus phases of ongoing oscillations on participants' perception individually. The result showed that, depending on the individual capacity of high-frequency time perception, certain phases of oscillations from beta to gamma bands predicted a higher probability of stimuli being perceived as simultaneous. The periods of those oscillations revealed a marginal linear correlation with the simultaneity thresholds and tended to be twice as much as they were. These findings suggest that the temporal perception is not associated with a unique oscillatory band, but general neural fluctuations in the brain. In particular, the consciousness could possibly serve as a lowpass filter during the explicit judgments of simultaneity.

\section{SYMPOSIUM 8: Submission 119}

\section{Distinct timing mechanisms in static and dynamic visual rhythm synchronization}

\author{
Yuelin $\mathrm{Liu}^{1}$, Tao $\mathrm{Xi}^{2}$, Yan Bao ${ }^{1}$ \\ ${ }^{1}$ School of Psychological and Cognitive Sciences, Peking \\ University, Beijing, China \\ ${ }^{2}$ Department of Cell and Developmental Biology, \\ University College London, United Kingdom
}

Sensorimotor synchronization refers to the coordination of body movements with an external rhythm such as tapping to a music beat. Previous studies focusing on auditory modality suggest that successful synchronization is restricted by the tempo of rhythmic stimuli. When the interstimulus interval is around 300-2000 ms, i.e., a fast tempo is involved, participants tend to tap before the onset of each stimulus, indicating a predictive timing mechanism. However, when the interstimulus interval becomes longer or the tempo is slow, prediction becomes difficult, and tapping starts to turn into a reaction mode with a large variance. The present study aimed to explore whether such tempo boundary exists in the visual modality, especially whether static and dynamic rhythmic stimuli show a difference. Synchronized tapping tasks were performed in young adults with three different types of rhythmic stimuli: tones, flashes and bouncing balls. The results showed a similar tempo boundary for synchronization to visual flash as to tones, but not for bouncing balls. No matter the tempo was fast or slow, the tapping always preceded the onset of the bouncing ball by approximately $20-50 \mathrm{~ms}$, and the variance of the tap-ball asynchronies stayed the same. These results suggest that different timing mechanisms are involved in static and dynamic visual rhythm synchronization. Continuously refreshed spatial information possibly benefits the prediction of incoming events even when the visual tempo is slow. Thus, distinct brain timing mechanisms are apparently involved in visual rhythm synchronization with and without spatial information. 


\section{SYMPOSIUM 8: Submission 134}

\section{Two distinct representational spaces of duration in sensory and motor timing}

\author{
Xиanyu Wang ${ }^{1,2}$, Shunyu Shi ${ }^{1}$ \\ ${ }^{1}$ School of Psychological and Cognitive Sciences, Peking \\ University, Beijing, China \\ ${ }^{2}$ Graduate School of Systemic Neurosciences, Ludwig- \\ Maximilians-University, Munich, Germany
}

Time is a critical aspect of spatial cognition: to navigate in physical space, organisms need to estimate the duration of time both in sensory perception and in motor planning, in both retrograde and anterograde manner. However, it is not yet clear how is the mental space of temporal duration arranged in the human brain. Many evidences point to the involvement of Basal Ganglion (BG) and Supplementary Motor Area (SMA) during timing; however, few studies have dealt with the alternative explanation that BG-SMA circuit might be relevant only for motor preparation that temporally coincides with the target interval. With the help of functional magnetic imaging, the brain activities during different task phases of duration reproduction were compared with perceptual and motor control. My study focused on the specific duration of $3 \mathrm{~s}$ of maximal reproduction precision which was originally discovered by Vierorth (1868). The results support the dissociation of two distinct neural circuits in duration processing: a bifunctional BG-SMA circuit that participates in both active timing and motor preparation, followed by a frontoparietal circuit for the duration working memory, consists of the dorsolateral prefrontal cortex (DLPFC) and the intraparietal sulcus (IPS). Combining our observations with evidence from animal studies, I propose that these two neural circuits correspond to two distinct mental spaces of duration, with different coding strategies and participating in different cognitive processes: The BG-SMA circuit encodes duration through a low-dimensional nonlinear accumulation, while the frontoparietal circuit stores duration in high-dimensional sparse coding.

\section{SYMPOSIUM 8: Submission 137}

\section{The 3 second time window in birdsongs: beyond} defining a territory

\author{
Shuai Chen ${ }^{1}$, Xinchi $\mathrm{Yu}^{2,3}$, Ernst Pöppel ${ }^{1,3}$ \\ ${ }^{1}$ School of Psychological and Cognitive Sciences, Peking \\ University, China \\ ${ }^{2}$ Department of Linguistics \& Neuroscience and Cognitive \\ Science Program, University of Maryland, College Park, \\ United States \\ ${ }^{3}$ Institute of Medical Psychology, Ludwig-Maximilians- \\ Universität, Munich, Germany
}

Background: Birdsongs (like those by Turdus merula) define a territory (Snow 2008). But birdsongs also have an aesthetic quality. It has been shown that aesthetic appreciations have also a temporal characteristic. For instance, poem lines have a duration of around 3 seconds during recitation; lines in music and shots in films may also fall in this 2-3-second window (Chen, Cai, \& Bao 2020). Poem appreciations seem to be shaped by this 3 -second window, as subjects like the best when poem lines are presented in 2-3-second time window (Yu \& Bao 2020; Zhao, Zhang, \& Bao 2018). Aims: It is yet unknown whether this temporal preference in aesthetic appreciation extends to birdsong appreciation, thus, going beyond intra-species spatial characteristics. The current experiment is aimed at testing this. Methods: Forty-seven subjects (20 male, age 19-31) made Likert ratings about 72 birdsong clips, constructed from 18 birdsongs (for details of the stimuli, see Roeske et al. 2020) and modified into four different durations (1600, 2800, 3200, $4000 \mathrm{~ms})$. Results: Aesthetic ratings reach a plateau after $3200 \mathrm{~ms}$. Interestingly, dividing the birdsong clips by original duration (shorter than $4000 \mathrm{~ms}, 4000-6000 \mathrm{~ms}$, longer than $6000 \mathrm{~ms}$ ), the subjects prefer the birdsong clips with the original duration of around 3 $\mathrm{s}$ the best. Conclusion: This study suggests that birdsong appreciation is shaped by the 3 second window. The preference of birdsong lines with an original duration of some $3 \mathrm{~s}$ supports the hypothesis of temporal information segmentation in humans.

\section{SYMPOSIUM 8: Submission 167}

\section{Multisensory Temporal Order Judgements: More salient Effect of Spatial Attention}

\author{
Mengtong Cai, Congyu Lin
}

\section{Peking University, China}

It has long been claimed that attended stimuli are perceived prior to unattended stimuli-a doctrine of "prior entry" being proposed by Titchener in 1908. Two parallel research directions have addressed how prior entry effect is produced by modality and spatial attention. However, whether spatial and modality information may jointly play a role in multisensory temporal order perception remains an open question. The present study aimed to examine how perception of multisensory information is affected when modality cue and spatial cue coexist exogenously by combining Posner's cueing paradigm with temporal order judgment task. A group of young participants were asked to judge the temporal order of two events (one is visual, one is auditory) proceeded by the presentation of a modality (visual vs. auditory) cue or a peripheral location (valid vs. invalid) cue. The results showed a non-significant modality effect, but a significant spatial cueing effect on audiovisual temporal order performance: an auditory dominance effect in temporal order judgement was magnified by spatial orienting. These observations suggest that multisensory temporal perception in high frequency time domain is presumably an automatic, stimulus-driven processing and more affected by flexible exogenous spatial attention.

\section{SYMPOSIUM 9: General abstract}

\section{The perception of space around us: from behavioral} to simulated data

Convenors: Luca Simione ${ }^{1}$ \& Onofrio Gigliotta ${ }^{2}$

${ }^{1}$ Institute of Cognitive Sciences and Technologies, CNR, Rome, Italy

${ }^{2}$ Department of Humanities, University of Naples Federico II, Italy

Space perception is one of the basic capabilities of our cognitive system. Identifying objects in space, computing their distance from the body, perceiving the body that moves towards an object are all fundamental abilities for life. Space perception involves a plethora of cognitive processes: visual perception, proprioception, auditory perception, time perception, planning, and movement perception, among others. Moreover, space includes both our body and the body of other individuals, such as space perception is implied also in social cognition. A very special place is the space surrounding the body, i.e., the peripersonal space, where most of the interactions with the external world happen. Lastly, as space perception implies awareness of the 
body and the other objects in the space, we can assume that it involves a very higher level of elaboration. Thus, understanding the basic principle of space perception is really important for the advance of cognitive sciences as a whole. However, due to the complexity of this construct and of the great implication it has for the cognitive system functioning, a multidisciplinary approach is needed, connecting multiple dominions and fields of science. To this aim, we proposed this symposium in order to offer a (virtual) place where researchers with different backgrounds can confront each other. In this symposium, experts from behavioral psychology, neuroscience, and computational science would present their work on space perception with the aim to encourage a cross discussion among applicants of different approaches in this important field. In particular, we will show how the integration of behavioral methods with neurocomputational approach to vision could improve the comprehension of experimental data. In fact, simulated neural and computational models allow to explain cognitive processes by simulating neurons and their connections, and then by replicating in simulation the phenomenon of interest. Such models go beyond both the first connectionist models, characterized by simple architectures and unrealistic neuronal models, and the machine learning approach, in which the task performance, e.g., in terms of classification accuracy, is the main outcome of the models at the expense of biological plausibility. In our view, instead, neurocomputational and simulated approaches should have the aim of replicating a cognitive process, such as space perception, by relying on biological data (e.g. a bioinspired connectivity pattern). Moreover, those models have another advantage, as they allow the study of neural lesions or damage on the perceptual system without involving the use of human patients and animal models. Lastly, we will show how high-level functions such as empathy and awareness could build on the scaffolding offered by very basic spatial abilities, and how viceversa the spatial perception could be affected by such high-level functions. For example, the simple capacity of computing the approximate distance of another person could dramatically change the way in which we perceive that person, e.g. if too close and threatening. Thus, understanding the basic principle of functioning of space perception would help us in conceiving new theoretical stances about complex cognitive processes and how they are implemented in the brain.

\section{SYMPOSIUM 9: Submission 189}

\section{From self to others, through the peripersonal space: how it is built compassion}

\section{Luca Simione ${ }^{1}$, Salvatore Gaetano Chiarella ${ }^{2}$ \\ ${ }^{1}$ Institute of Cognitive Sciences and Technologies, CNR, Rome, Italy \\ ${ }^{2}$ Department of Psychology, Sapienza, University of Rome, Italy}

Background: Peripersonal space (PPS) is the functionally reachable space closely surrounding the body. Crucially, its boundaries are not fixed, but it is shaped by the interactions between the body and objects in the environment, for instance by social interactions. Recent studies suggest that mindfulness meditation can modulate inter-subjective social behaviors and self-other representations. They also showed that mindfulness can intensify empathy and compassion. Thus, mindfulness seems to modulate both self-other integration and PPS. Aims: We investigated the relationship between mindfulness and PPS, in order to understand how mindfulness shapes the extent of personal psychological boundaries. We hypothesized that a modulation in cognitive domain, such as empathy and compassion, could be driven in bottom-up fashion by changes in self and bodily representations. Methods: We conducted a qualitative, critical review of the papers investigating the modulation of PPS in experiments including or manipulating mindfulness (e.g. with meditation induction). Results: Phenomenological studies (e.g. Ataria et al. 2015; Berkovich-Ohana et al. 2013) showed that mindfulness modulates the experience of the self by impacting the sense of boundaries that can be extended toward the external world. Also experimental studies showed that PPS can be expanded or diminished by meditating (e.g. Lindahl \& Britton 2019). We discuss the reported experimental results in light of the effects of mindfulness on empathy and compassion. Conclusion: We thus proposed how the changes observed in social domains as a consequence of practicing mindfulness could be driven by low-level changes in bodily-sensory representation.

\section{SYMPOSIUM 9: Submission 192}

\section{Does sensory memory depend on visuospatial attention?}

\author{
Salvatore Gaetano Chiarella ${ }^{1}$, Luca Simione ${ }^{2}$
}

${ }^{1}$ Department of Psychology, Sapienza, University of Rome, Italy

${ }^{2}$ Institute of Cognitive Sciences and Technologies, CNR, Rome, Italy

Background: Sensory (iconic) memory and visual working memory (VWM) are key components of the classical modal model of memory. Sensory memory and VWM are also central in important theories of human visual consciousness, such as the global workspace and recurrent processing theory. Although the role of selective attention in transferring information in VWM is well-established, the relationship between selective attention and sensory memory is still a debated issue. Aims: We aimed to investigate the effect of visuospatial attention on sensory memory. We hypothesized that sensory memory representations do not require attention, while attention was necessary to consolidate such representations in VWM. Methods: In two behavioural experiments, we used a change detection paradigm with delayed cueing of the target for report, with high (80\%), equal (50\%), and low $(20 \%)$ priority objects, investigating the relationship between sensory memory (iconic memory and fragile-VSTM) and selective attention. We also designed a neurocomputational simulated model to replicate our behavioural results, in order to further understand the relationship between visuospatial attention and memory representations. Results: Results supports early findings showing an independence of sensory memory from selective attention, while an attentional bias, mainly modulated by suppressive mechanism, occurs at longer delays. Our simulations supported the distinction between local recurrent circuits, that maintained sensory representations without attention, and global recurrent circuits, for which attention was needed. Conclusion: Through an integrative approach between behavioural data and neurocomputational simulations we propose an updated taxonomy of theories of visuospatial attention and consciousness.

\section{SYMPOSIUM 9: Submission 199}

From learning to search to finding what others miss: developing a theory of mind

Dimitri Ognibene ${ }^{1,2}$, Francesca Bianco ${ }^{2}$, Letizia Marchegiani ${ }^{3}$

${ }^{1}$ Università Milano-Bicocca, Italy

${ }^{2}$ University of Essex, United Kingdom

${ }^{3}$ Aalborg University, Denmark 
In the last 10 years, with the advent of modern deep learning methodologies, substantial performance improvement has been observed in artificial perception systems. However, the common solution to deal with the sensory limits (e.g. occlusions and limited FOV) through the increase in the number and types of sensors resulted in an escalation of costs, weight, and power demands, while important information can still be missed (e.g. occlusions). Active perception mechanisms, that enable to dynamically deal with sensory limits, have received less attention. Yet, it has been recently shown that such mechanisms, which select part of the input also through spatial relations, may substantially improve learning performance and even enable online adaptation to new environments. Active perception plays a crucial role also when interacting with other agents, who may have a different field of view or occlude relevant information. We here present a line of study about learning to find relevant information in solo tasks and during social interactions. We propose that learning combined with self-observation while performing such tasks may be at the base of the development of the flexible theory of mind capabilities.

\section{SYMPOSIUM 9: Submission 201}

\section{A neurorobotic model of stress-induced pseudoneglect}

\author{
Onofrio Gigliotta $^{1}$, Michela Ponticorvo ${ }^{1}$, \\ Orazio Miglino $^{1,2}$, Paolo Bartolomeo ${ }^{3}$ \\ ${ }^{1}$ University of Naples Federico II, Italy \\ ${ }^{2}$ ISTC, CNR, Italy \\ ${ }^{3}$ Sorbonne Université, Institut du Cerveau-Paris Brain \\ Institute-ICM, Inserm, CNRS, AP-HP, Hôpital de la Pitié- \\ Salpêtrière, Paris, France
}

Background: We have recently shown that the stress related to the 2020 Italian lockdown was able to increase pseudoneglect, a wellknown spatial leftward bias, in a cancellation task (Somma et al. 2021). The cancellation task used in that work had been developed by Gigliotta and colleagues (Gigliotta, Miglino, and Bartolomeo 2017) to test the ability of different neural architectures, controlling the behavior of a population of neurorobots, to display pseudoneglect. Aims: Stress and brain laterality have been studied in animal models as well as in humans. Their relationship might result from the effect of stress-related hormones to information transfer between the hemispheres across the corpus callosum. Specifically, Ocklenburg and colleagues (2016) proposed that stress may decrease neural transmission between hemispheres. Methods: We manipulated the callosal connections of our pseudoneglect neurorobotic model, to assess the validity of t the Hausmann-Gunturkun hypothesis. Results: Preliminary results show that neural transmission between hemispheres can indeed modulate the amount of leftward bias in our model. Conclusion: Neurorobotics allows us to implement an artificial neurocontroller for a simulated body, and thus to test hypotheses that are difficult to tackle in natural contexts. In this work, we exploit the potentialities of this approach, by testing a specific hypothesis on the role of interhemispheric transfer of information in modulating pseudoneglect.

\section{SYMPOSIUM 10: General abstract}

\section{Spatial attention performance assessed by the ANT on children and young adults: Its association with age, intelligence and impulsiveness}

\author{
Convenors: Chiara Saracini ${ }^{1,2} \&$ Boris Lucero $^{1}$ \\ ${ }^{I}$ The Neuropsychology and Cognitive Neurosciences \\ Research Center (CINPSI Neurocog), Faculty of Health \\ Sciences, Universidad Católica del Maule, Talca, Chile \\ ${ }^{2}$ Centro de Investigación de Estudios Avanzados del Maule \\ (CIEAM), Universidad Católica del Maule, Chile
}

Attention is a multidimensional ability known to serve different topdown cognitive functions, working at the same time as an organic system (Posner and Fan 2008). According to the Posner model's definition of the Attentional process, three specialized brain networks are associated with different neuroanatomically-based cognitive functions of alerting, orienting, and executive control. These abilities are quite relevant for optimal cognitive performance and are associated with other important networks' correct functioning (the salience network, the default mode network, and the dorsal and ventral attention networks). These three networks (alerting, orienting, and executive control) can be assessed by the Attentional Networks Task (ANT; Fan et al. 2002), which is a modification of a simple flanker task with the addition of spatial cues before the target stimuli, measuring the response's reaction time and accuracy. Visual-spatial attention is usually unaltered through the life span and resistant to aging, and almost entirely developed at 6 years old for the non-clinical population. However, some studies have reported individual differences associated with cognitive style and other executive functions. Spatial attention has been explored, for example, in different personality traits (e.g., impulsiveness), cognitive functions (mainly related to attentional processes and other executive functions), and intelligence (IQ). This symposium will have a focus on the assessment of the attentional networks through the ANT or modifications of this test (e.g., ANTIFruits for adults, the ANTI-Vigilance). Studies from Italy and Chile will present results of behavioral and electrophysiological measures and address individual differences and cognitive-related topics in diverse populations. One of the single contributions will present an ANT's children-friendly version, which has been used to explore children's attentional performance, showing that attentional skills improve between 3 and 4 years of age, with no significant further improvements. A second contribution will present the association between ANT's performance and intelligence in 12-13-year-old children, analyzing cognitive processing efficiency using a global measure of brain connectivity (the weighted Symbolic Mutual Information, wSMI) from EEG recordings. It will show preliminary results about the relation of cognitive efficiency with high IQ and improved performance in the ANT task. Another contribution will discuss the adolescents' (from 10 to 19 years old) performance in the ANTI-V task, allowing the conclusion that all the attentional networks seem to continue to develop during adolescence. However, after age 15, adolescents adopt a more conservative response strategy and increase the monitoring of self-errors, while a low level of vigilance seems to harm alerting and orienting abilities. Another contribution will present the significant association between impulsiveness and the ANT performance on young adults (from 20 to 24 years old), especially on the Executive Control network, with a mild effect of age on the Alerting network. Finally, the last contribution's aim will be to analyze the agerelated change of hemispheric specialization of attentional networks in elderly participants to clarify the decrease in attentional functioning in elderly/older age. It will show data that support the hypothesis of a hemispheric asymmetry reduction in this age-group. 


\section{SYMPOSIUM 10: Submission 130}

\section{Impulsiveness influence on Attentional Networks in young adults}

Chiara Saracini ${ }^{1,2}$, Boris Lucero ${ }^{1}$, Francisco Ahumada ${ }^{1,3}$, Maria Teresa Muñoz-Quezada ${ }^{l}$

${ }^{1}$ The Neuropsychology and Cognitive Neurosciences Research Center (CINPSI Neurocog), Faculty of Health Sciences, Universidad Católica del Maule, Talca, Chile

${ }^{2}$ Centro de Investigación de Estudios Avanzados del Maule (CIEAM), Universidad Católica del Maule, Chile

${ }^{3}$ Doctorate in Psychology, Faculty of Health Sciences, Universidad Catolica del Maule, Chile

Background: Impulsive behavior affects cognitive tasks performance, particularly attentional ones. High impulsiveness is generally related to difficulties inhibiting a prepotent response in the stop-signal task. This trait could have a direct influence on the temporal preparation of motor responses and also involves a "temporal orienting of attention", associated with the endogenous orienting network. Alerting, orienting, and executive control attentional networks are assessed by the ANT Test, developed on the basis of Posner model of attention. In literature, while there is agreement on the alerting and executive control networks as being affected by a number of personality traits, cognitive styles and clinical conditions, there are mixed results about the orienting network being either a relatively stable one (even through the life span) or impaired in clinical populations (e.g., autism, ADHD, Parkinson, posttraumatic stress disorder, anxiety), and there is evidence of individual differences in spatial attention functioning due to different cognitive styles and information processing. Aims: To examine the impact of impulsiveness on the attentional networks. Methods: A sample of 88 students responded to the ANT and the BIS-11 Barratt's impulsivity scale. Results: Regressions show that alerting network efficiency is predicted by the cognitive impulsivity subscale of BIS-11. Executive control is associated with both motor (directly) and non-planning (inversely) impulsivity scales. Conclusion: Cognitive, motor and nonplanning impulsiveness BIS-11 subscales are associated with alerting and executive control attentional networks. The efficiency of the orienting network seems to be unrelated to any impulsiveness score.

\section{SYMPOSIUM 10: Submission 140}

\section{Assessing the attentional networks in children: a child version of the ANTI}

Maria Casagrande ${ }^{1}$, Andrea Marotta ${ }^{2}$, Diana Martella ${ }^{3}$, Elisa Volpari ${ }^{4}$, Francesca Agostini ${ }^{5}$, Francesca Favieri ${ }^{5}$, Giuseppe Forte ${ }^{5}$, Monica Rea ${ }^{1}$, Rosa Ferri ${ }^{1}$,

Vito Giordano ${ }^{6}$, Jasmine Giovannoli ${ }^{5}$

${ }^{I}$ Dipartimento di Psicologia Dinamica e Clinica, Sapienza University of Rome, Italy

${ }^{2}$ Departamento de Psicología Experimental, Universidad de Granada, Granada, Spain

${ }^{3}$ Instituto de Estudios Sociales y Humanisticos, Universidad Autónoma de Chile, Santiago de Chile, Chile

${ }^{4}$ Istituto di Istruzione Superiore V. Bachelet, Abbiategrasso, Italy

${ }^{5}$ Dipartimento di Psicologia, Sapienza University of Rome, Italy
${ }^{6}$ Department of Pediatrics and Adolescent Medicine, Division of Neonatology, Pediatric Intensive Care and Neuropediatrics, Comprehensive Center for Pediatrics, Medical University of Vienna, Austria

Attention involves three functionally and neuroanatomically distinct neural networks: alerting, orienting, and executive control. This study aimed to analyze the development of attentional networks in children aged between 3 and 6 years using a child-friendly version of the Attentional Network Test for Interaction. The sample included eightyeight children divided into four age groups: 3-year-old, 4-year-old, 5-year-old, 6-year-old children. The results of this study indicated that between 4 and 6 years, there are no significant changes in attentional networks. Instead, between 3 and 4 years of age, children significantly improve all their attentional skills.

\section{SYMPOSIUM 10: Submission 148}

\section{wSMI, Intelligence, and attentional performance on children: Preliminary results}

Boris Lucero ${ }^{1}$, Andres Canales-Johnson ${ }^{1,2}$, Chiara Saracini $^{1,3}$, Maria-Teresa Muñoz-Quezada ${ }^{1}$

${ }^{1}$ The Neuropsychology and Cognitive Neurosciences Research Center(CINPSI Neurocog), Universidad Católica del Maule, Chile

${ }^{2}$ Department of Psychology, University of Cambridge, United Kingdom

${ }^{3}$ Centro de Investigación de Estudios Avanzados del Maule (CIEAM), Universidad Católica del Maule, Chile

Background: Intelligence performance is associated with better brain functioning efficiency. Cognitive resources are optimized so better responses are achieved with less energy consumption. A measure of brain efficiency is functional connectivity. To date, no studies have addressed the association between IQ, connectivity, and spatial attentional task performance in children. Also, none have measured the weighted symbolic mutual information (wSMI) to explore the brain electrophysiological activity correlates and their relation with IQ and the attentional network task (ANT). Aims: To examine how each attentional network (alerting, orienting, and inhibition) can benefit from the optimized efficiency related to IQ. Methods: A preliminary sample $(\mathrm{N}=20)$ of children $(12-13$ years old, where the IQ and ANT performance has reached a stable stage of development) were assessed through the Wechsler Intelligence scale. Also, they were examined through EEG recording during the ANT administration. The ANT measures visual attention through three networks: alerting, orienting, and inhibition. Behavioral responses were measured through reaction time and accuracy. The EEG was analyzed using the wSMI, computing the extent to which the signals from two electrodes present joint non-random fluctuations, obtaining a global measure of shared mutual information. Hypothesis: IQ score is directly associated with brain efficiency as evaluated by the wSMI connectivity measure on each attentional network studied. Results: Concerning wSMI, only the orienting response correlates significantly with better IQ. Conclusion: Better IQ performance of children, in terms of brain functioning efficiency, is specifically related to spatial attention. 


\section{SYMPOSIUM 11: General abstract}

Multisensory space and action: state-of-the-art research and future perspectives of voluntary and defensive motor behavior in peripersonal space

\section{Convenors: Claudio Brozzoli, Alessandro Farnè \& Ivan Patanè}

\section{Impact, Lyon Neuroscience Research Centre, Neuro-im- mersion, France}

When interacting with objects and other people, the brain needs to locate our limbs and the relevant sensory information surrounding them. Studies on monkeys showed that information from different sensory modalities converge at the single cell level within a set of interconnected multisensory fronto-parietal areas. It is largely accepted that this network allows for multisensory processing of the space surrounding the body (peripersonal space), whose function has been linked to the sensory guidance of actions. However, whether appetitive and defensive movements are supported by the same multisensory mechanisms and follow the same rules remains unclear. Are reach goals coded by peripersonal space circuitry during action planning? Are objects around someone else's body and another person's actions encoded by the same or different mechanisms underlying the observer's peripersonal space? Is peripersonal space supporting associative learning of threats in body-part-centered coordinates? Is peripersonal space equally supporting appetitive actions such as grasping? The aim of this symposium is to discuss these questions by focusing on multidisciplinary findings about the processing of the space near the body in humans and non-human primates, offering a convergent view of its functions and underlying neural mechanisms. Experts in the field will discuss the role of parietal and premotor areas of the non-human primate brain in encoding reach targets. Previous studies showed that those areas use coordinate systems anchored to different parts of the subjects' body, such as hand position, often modulated by head orientation and gaze direction. New results presented in this symposium show that neuronal activity in the same areas dynamically changes with task demands, also encoding object-relative spatial information, independent of object location and object size, or egocentric information. The symposium will present also investigations of space-constrained representations in the freely moving monkey. They will show that processing of observed objects and others' action depends on largely overlapping neural substrates within the network of parieto-frontal brain regions devoted to the planning and control of manual actions, encompassing the anterior intraparietal area (AIP), the ventral premotor area F5 and the mesial presupplementary motor area F6, up to the single neuron level. Behavioral data on humans confirm that the dynamic properties of the representation of peripersonal space are linked both to appetitive and defensive behavior. The symposium will discuss results showing that manual action planning triggers handcentered changes in multisensory perception which precede and accompany action unfolding. New evidence will be provided that peripersonal space contributes to associative fear learning, crucial for defensive behavior, by encoding valence-event associations in bodypart-centered coordinates. We will suggest that evolution has provided the brain with a clever tool for representing visual information around body parts. We will contend that the peripersonal space representation, is a multisensory motor interface that allows for both appetitive and defensive interactions with the world around us.

\section{SYMPOSIUM 11: Submission 156}

Associative fear learning in hand-centered coordinates

Alessandro Zanini ${ }^{1,2}$, Romeo Salemme $e^{1,2,3}$, Alessandro Farne $e^{1,2,3,4}$, Claudio Brozzoli ${ }^{1,2,3,5}$

${ }^{1}$ ImpAct Team, Lyon Neuroscience Research Centre, INSERM U1028, CNRS UMR5292, Lyon, France

${ }^{2}$ University Claude Bernard Lyon I, Lyon, France

${ }^{3}$ Hospices Civils de Lyon, Neuro-immersion-Mouvement et Handicap, Lyon, France

${ }^{4}$ Center for Mind/Brain Sciences, University of Trento, Rovereto, Italy

${ }^{5}$ Department of Neurobiology, Care Sciences and Society, Aging Research Center, Karolinska Institutet, Stockholm, Sweden

Background: Space coding affects perception of stimuli associated with negative valence: threatening stimuli presented within the peripersonal space (PPS) speed up behavioral responses compared to non-threatening events. However, it remains unclear whether the association stimulus-negative valence is acquired in a body-part-centered reference system, a main feature of the PPS coding. Aims: Demonstrate that associative fear learning might take place in handcentered coordinates: once the stimulus-negative valence association has been established within the hand-centered PPS, the threat is no longer encoded within an egocentric reference frame, but in relation to the position of the hand itself. The movement of the hand would then involve the remapping of this association. Methods: Through a Pavlovian fear-learning paradigm, we associated a visual stimulus (conditioned stimulus, CS) with an aversive one (electrocutaneous shock) applied on the right hand only when the CS was projected close $(\mathrm{CS}+)$ but not far from it (CS-). In test phase, the hand was displaced close to the position of visual stimulation previously never associated with the electrocutaneous shock. Results: In learning phase, skin conductance responses (SCRs) showed a successful learning of the negative value of the $\mathrm{CS}+$, with a higher anticipatory fear response associated with it. Noteworthy, when participants moved their hand in a novel position, higher SCR was associated with visual stimuli projected close to this new position, previously not associated with tactile shocks. Conclusion: These results indicate a hand-centred mapping of the conditioning effect, confirming the possibility of establishing associative learning bonds in body-part-centred coordinates.

\section{SYMPOSIUM 11: Submission 175}

Task-dependent ego- and allocentric encoding in monkey frontoparietal cortex

Bahareh Taghizadeh ${ }^{1,5}$, Ole Fortmann ${ }^{1,2}$, Alexander Gail ${ }^{1,2,3,4}$

${ }^{1}$ Cognitive Neuroscience Laboratory, German Primate Center, Göttingen, Germany

${ }^{2}$ Faculty of Biology and Psychology, University of Göttingen, Göttingen, Germany

${ }^{3}$ Bernstein Center for Computational Neuroscience, Göttingen, Germany

${ }^{4}$ Leibniz ScienceCampus Primate Cognition, Göttingen, Germany

${ }^{5}$ School of Cognitive Science, Institute for Research in Fundamental Sciences, Tehran, Iran 
Humans utilize both egocentric (subject-dependent) and allocentric (subject-independent) spatial information for target localization during goal-directed movement behavior. The brain regions and neural mechanism for constructing allocentric frames of reference in primates are still under debate. Egocentric spatial encoding is a wellknown property of brain areas along the dorsal pathway, monkey parietal reach region (PRR) and dorsal premotor cortex (PMd) encode spatial parameters for planning upcoming reach movements in different egocentric coordinate systems. Yet, natural reaches are typically directed towards extended physical objects. Not only their location, but also their spatial structure guides reach planning. We ask if planning activities in PRR and PMd can also encode object-centered (allocentric) reach goal information. Monkeys planned reaches towards different sites on an object. During visual memory, they could memorize an on-the-object position; during reach planning, they had to plan a reach to this position after re-occurrence of the object at a potentially different position and with potentially different size. In both brain areas, we find (1) predominant object-centered encoding during visual memory, which (2) changes to predominant egocentric encoding during reach planning corresponding to changing cognitive demands, and which (3) is invariant to object position and size. Such flexible and cognitively controlled encoding within local brain regions contrasts the idea of task-independent functional segregation between processing pathways and is particularly suitable for variable-demand interaction with dynamic environments.

\section{SYMPOSIUM 11: Submission 181}

\section{Space-constrained cortical processing of objects and actions in the monkey}

\section{Luca Bonini}

Dept of Medicine and Surgery, University of Parma, Italy The neural processing of objects around us involves visual areas as well as the parieto-frontal sensorimotor system, which extracts from the object's sensory description the various motor possibilities that an observer has to interact with it, including approaching and defensive actions. Recently, we have proposed observed actions performed by others constitute "social objects" to which the same principles revealed for visuomotor coding of objects can apply. We comparatively investigated the encoding of objects and others' actions in a network of parieto-frontal brain regions devoted to the planning and control of manual actions, encompassing the anterior intraparietal area (AIP), the ventral premotor area F5 and the mesial presupplementary motor area F6. The results of these studies indicate that the processing of observed objects and others' action depends on largely overlapping neural substrates, up to the single neuron level, and it is remarkably constrained to a body-centered reference frame. Our findings suggest that visuo-motor remapping of physical and social stimuli on the motor system is primarily aimed at selecting potential motor actions, to be eventually turned into overtly executed individual or social behaviors depending on the contextual situation. The most recent and ongoing research in our group is elucidating the cortical dynamics occurring along these parieto-premotor circuits, suggesting new roles for specific neuronal classes and new avenues in the investigation of space-constrained representations in the freely moving monkey.

\section{SYMPOSIUM 11: Submission 185}

\section{Action-dependent modulations of multisensory perception in peripersonal space}

Ivan Patanè, Claudio Brozzoli, Alesandro Farnè

Impact, Lyon Neuroscience Research Centre, Neuro-immersion, France

Peripersonal space (PPS) is a highly plastic representation that integrates tactile and visual stimuli presented on, and close to, the body. This system seems to contribute to the efficient guidance of movements and now there's evidence of a prominent role of PPS in the control of action. In support of this view, we showed that PPS plastic changes occur before and during action. The results from our study reveal that visual and tactile information strongly interact already during the planning phase of action and this visuo-tactile interaction is further enhanced during subsequent movement phases. Such a visuotactile interaction is ideally suited to planning and guiding actions. Most of our action take place in social interactions, and PPS is also sensitive to the social features of interactions. In a new study, we probed this sensitivity of PPS to a so far unexplored social dimension: ownership. The results indicate that, whether considered to be as individual or shared property, ownership of an object is critical for the PPS modulations during action execution and observation. Visuotactile interaction emerged only when the object belonged to the acting participant. Interestingly, a similar effect was found when observing another person acting on their own object. In a follow-up experiment, we investigated PPS plastic changes when property of the object was shared between the two agents. In this case, visuo-tactile interactions emerged not only when acting in first person, but also when observing the peer acting upon the shared object.

\section{SYMPOSIUM 12: General abstract}

\section{The space around the body and its relationship with sensorimotor and social factors: current questions (and some answers) for future directions}

\author{
Convenors: Alessandro Farnè ${ }^{l}$, Fadila Hadj-Bouziane ${ }^{1}$, \\ Tina Iachini $^{2}$ \& Gennaro Ruggiero ${ }^{2}$ \\ ${ }^{1}$ Impact, Lyon Neuroscience Research Centre, Neuro-im- \\ mersion, France \\ ${ }^{2}$ Department of Psychology, University of Campania L. \\ Vanvitelli, Italy
}

The portion of space surrounding our body, where we can act in the here and now, is our first interface with the physical and social world. Our brain represents this space of interaction with objects and human beings through dedicated mechanisms. There is much debate in the literature about the nature of this space, in particular, whether there is a single representation that supports multiple functions (i.e. action with objects, defense, social interaction) or whether there are multiple representations each specialized for one function. One potential difficulty is to congregate results stemming from several experimental approaches that have been employed to delineate this portion of space surrounding our body, leading do different concepts such as the peripersonal space, the reaching space, or the comfort space. This debate has raised important questions such as: Can we trace a common evolutionary mechanism underlying the social and sensorimotor spaces and if so when do the differences begin? Are we facing different task constraints, or different spaces? Are we addressing merely different labels, or truly separate concepts? The aim of this symposium was to discuss these questions by focusing on the weight of 
social and action-related factors in the representation of near body space to unveil the multiplicity of its identities and functions. In this symposium, several experts in the field will contribute to elucidate these aspects. They will discuss how emotional faces influence perceptual abilities close or far from the body in healthy subjects. Beyond behavior, the objective is to consider body and brain responses under different emotional contexts to clarify the impact of these social factors on the representation of the close space surrounding our body. They will also relate on the effects that sensorimotor interactions between people have in modulating brain activations coding for the peripersonal space representation that is shared by two individuals and discuss how the physiological component, indexed by the skin conductance response, contributes to define the interpersonal social space regulation in a clinical population of children with autism.

\section{SYMPOSIUM 12: Submission 57}

\section{Physiological correlates of social space in childhood autism spectrum disorders}

\author{
Michela Candini ${ }^{1}$, Gianmarco Mellini ${ }^{1}$ \\ Simone Battaglia ${ }^{1,2}$, Virginia Giuberti, \\ Giuseppe di Pellegrino ${ }^{1,2}$, Francesca Frassinetti ${ }^{1,4}$ \\ ${ }^{1}$ Dipartimento di Psicologia "Renzo Canestrari", Univer- \\ sità di Bologna, Italy; \\ ${ }^{2}$ Centro Studi e Ricerche in Neuroscienze Cognitive- \\ CsrNC, Cesena, Italy; \\ ${ }^{3}$ Centro Autismo-Programma Autismo AUSL di Reggio \\ Emilia, Italy; \\ ${ }^{4}$ Istituti Clinici Scientifici Maugeri-IRCCS di Castel \\ Goffredo, Castel Goffredo, Mantova, Italy.
}

Background: Interpersonal space (IPS) is the area that individuals maintain between themselves and others and that, when it is violated, cause discomfort. The IPS's extent depends on others' behaviour, and the closeness with an unknown person increases the skin conductance response (SCR), linking behavioral and autonomic components of IPS. Aims: We investigate whether the deficit in IPS regulation reported in children with Autism Spectrum Disorders (ASD), is associated with an altered SCR to social proximity. Methods: Children with typical development (TD) and children with ASD performed two Experiments. In Experiment 1, participants stopped an unknown adult (confederate), who moved toward to or away from them, when they felt comfortable with other's distance (comfortdistance). In Experiment 2, the phasic participants' SCR was recorded when the confederate moved and briefly stopped at five spatial positions, simulating an approach/withdrawal movement. Results: We found that ASD children preferred a larger IPS and exhibited higher SCR than controls. Interestingly, in TD and ASD children, the SCR increased when the confederate was closer to them, reflecting the ability to detect a possible threat around the body. However, in TD children, this effect was observed in Approaching but not in Withdrawal condition, whereas in the ASD children the SCR was not modulated by the confederate's movement direction. This result can be ascribed to a deficit in ASD children in anticipating the movement direction performed by the confederate. Conclusion: We reveal that impairment of both physiological responses and predictive mechanisms contribute to abnormal IPS regulation found in autism.

\section{SYMPOSIUM 12: Submission 65}

\section{Defensive function towards social-emotional stimuli approaching our body}

\section{Gennaro Ruggiero, Tina Iachini}

Department of Psychology, University of Campania L. Vanvitelli, Italy

The need to provide an area of safety around the body encompasses the processing of both social and physical stimuli and requires efficient responsiveness. Therefore, this spatial interface with objects and people can be defined as an action possibility-based sensorimotor space. Its extent, while flexible, is within a reaching space in which we can act in the here and now. It also constitutes a space with a social-emotional valence, as our comfort space with others that is emotionally marked. Previous evidence suggests that the encoding of this near space reflects a fundamental defensive function that engages anticipatory and attentional mechanisms. We aim to understand the defensive function of the near-body area by comparing psychophysiological reactions to positive, negative and neutral virtual stimuli in the social space (i.e. the comfort area) and the action space (i.e. the reaching area). Participants had to determine the comfort-distance (at which people felt comfortable with the other's proximity) and the reaching-distance (at which people thought they could touch the other) from stimuli. Psychophysiological reactions towards emotional stimuli (i.e. skin conductance or SCR and interbeat intervals or IBI) were recorded and behavioural distances were measured. The results showed an increase in SCR amplitude, a decrease in IBIs, and a widening of distance in response to threatening stimuli in both tasks, although IBI was more sensitive to the emotional valence in reaching than comfort space. The theoretical meaning of the findings will be discussed in terms of defensive functions.

\section{SYMPOSIUM 12: Submission 71}

\section{Behavioral, physiological and neural signatures of PPS in social contexts}

\section{Fadila Hadj-Bouziane, Audrey Dureux}

\section{Lyon Neuroscience Research Center, ImpAct Team University of Lyon 1, France}

Background: Accumulating evidence indicates that the peripersonal space (PPS) constitutes a privileged area for efficient processing of proximal stimuli, allowing to flexibly adapt our behavior to the environment, an environment often involving others conspecifics. In the case of conspecifics, the emotional facial expressions they convey play an important role in social interactions. Aims: Whether and how behavioral, physiological and neural signatures of PPS relate to each other in emotional contexts remains, though, elusive. Methods: Here, we addressed this question in an immersive virtual reality environment where participants discriminated male from female faces depicting different emotions and presented at different distances $(50-300 \mathrm{~cm})$. We measured their accuracy, reaction times, pupil dilation and heart rate. Results: Results showed facilitation of participants' performances (i.e. faster response time) when faces were presented in close compared to far space, even when controlling for retinal size across distances. These behavioral effects were accompanied by significant modulation of participants' physiological indexes when faces were presented in PPS. Finally, social stimuli in close space modulated brain activity within the face network and parieto-premotor PPS network. Interestingly, these responses were affected by the features of the faces such as the emotional valence. Conclusion: Together, these 
findings suggest that both external and internal signals contribute in shaping PPS representation. We propose that an adjustment of brainbody interactions may play a critical role in adapting behavioral responses depending on the environment within PPS and may participate in regulating distances in social interactions.

\section{SYMPOSIUM 12: Submission 150}

\section{Brain activations for objects near two persons' hands after motor interactions}

\author{
Ivan Patane ${ }^{I}$, H. Henrik Ehrsson ${ }^{2}$, Claudio Brozzoli ${ }^{1}$ \\ ${ }^{1}$ Impact team-CRNL, Lyon, France \\ ${ }^{2}$ Department of Neuroscience, Karolinska Institutet, \\ Stockholm, Sweden
}

Interacting with people requires a shared representation of space (Brozzoli et al. 2014). Single-cell recordings have reported that parietal visuotactile neurons discharge for objects near a monkey's own hand and near the hand of another individual (Ishida et al. 2010). In humans, we identified neuronal populations in the human PMv that encode the space near both one's own hand and another person's hand (Brozzoli et al. 2013). The observer's brain therefore constructs a body-centered representation of the space around others similar to one's own peripersonal space representation (Brozzoli et al. 2011). Here, through functional magnetic resonance imaging, we investigated how activity of neural populations coding for the shared peripersonal space representation is modulated after a motor interaction with another person. In four different blocks during functional acquisition in the scanner, the hand of the participant and the hand of the interacting person could be orthogonally present or absent so to result in four different conditions: nobody, self, other or both present. A 3D object was presented in front of the participant $(N=26)$ always in the same reachable position, equidistant from the hands of the self and the other, if present. The contrast between the activations elicited by the object across different conditions allowed to test for stronger premotor responses to both-compared to self- and other-specific peripersonal space representations. These results can help to understand how the neural mechanism underlying a shared peripersonal space representation can support motor interactions with others by coding sensory events in a common spatial reference frame.

\section{SYMPOSIUM 12: Submission 180}

\section{The role of peripersonal space in social interactions}

\section{Yann Coello, Alice Cartaud}

SCALab-Sciences Cognitives et Sciences Affectives, Lille, France

The peripersonal space is an adaptive and flexible interface between the body and the environment that fulfills a dual-motor function: preparing the body for voluntary object-oriented actions to interact with incentive stimuli and preparing the body for defensive responses when facing potentially harmful stimuli. Peripersonal space is thus an abstract representation of the space surrounding the body whose limit corresponds to the maximum distance at which we can physically interact with manipulable objects. We will provide arguments for the sensorimotor rooting of the peripersonal space representation and highlight the variables that contribute to its flexible and adaptive characteristics. We will also demonstrate that the peripersonal space represents a mediation zone contributing to not only the control of goal-directed actions but also the organization of the social life. The data presented will lead us to the proposal of a new theoretical framework linking the peripersonal action space and the interindividual social space, i.e., the physical distance we naturally use to interact socially with conspecifics.

\section{SYMPOSIUM 13: General abstract}

\section{The development of a multisensory sense of space}

\author{
Convenors: Alice Bollini \& Monica Gori
}

\section{Unit for Visually Impaired People, Istituto Italiano di Tecnologia, Genoa, Italy}

The ability to encode space is a crucial point of interacting with the external world. To plan and carry out the actions in response to environmental events, it is necessary to continuously estimate, encode, and integrate the spatial information into a stable representation of the environment. These spatial representations must update the spatial locations of events to adapt to an unstable moving environment. During development, children's brains have to integrate the information from different sensory modalities to build a reliable external world representation. Integrating different spatial representations to encode space is a fundamental step to acquire during infancy and childhood 1,2. The recent literature has seen an increasing interest in developing space ability in early life. It has been demonstrated that vision plays a key role in the acquisition of spatial knowledge. Indeed, vision allows perceiving multiple stimuli simultaneously. It represents the most accurate sense for spatial information, allowing an exhaustive representation of the surrounding environment in a single frame $3-5$. Here we want to focus on how children build a stable representation of space with particular attention to how the different sensory modalities communicate with each other and particularly the role of the vision in shaping the world's spatial representation. The symposium brings together experts who have investigated the development of spatial abilities in children and different perspectives. Monica Gori will review how the absence of one sensory input affects the development of different modalities and technologies based on multisensory feedback associated with body movements. These technologies combine an auditory or visual cue with participants' movements improving spatial abilities and social interaction. Irene Senna will focus on how cataract-treated individuals can develop congruent spatial representations after surgery after many years of visual deprivation. Chiara Martolini will discuss the role of vision in the development of spatial coding, emphasizing the developmental delay of children with visual impairments. Giulia Orioli will present her results about how infants integrate the visual ad tactile experience to build a situated representation of their body in the surrounding space. Finally, Irene Valori will explore the developmental differences related to the ability to locate ourselves in space relying on vision and proprioception, in real and virtual environments, in children and adults typically developed and with autism spectrum disorders.

\section{SYMPOSIUM 13: Submission 31}

\section{Development of spatial coding skills in visually impaired and blind children}

\author{
Chiara Martolini ${ }^{1}$, Giulia Cappagli ${ }^{2}$, Sabrina Signorini ${ }^{1}$, \\ Monica Gori ${ }^{2}$ \\ ${ }^{1}$ Center of Child Neuro-Ophthalmology, IRCCS Fon- \\ dazione Istituto Neurologico Nazionale C. Mondino, Pavia, \\ Italy \\ ${ }^{2}$ Unit for Visually Impaired People, Istituto Italiano di \\ Tecnologia, Genova, Italy
}


Vision is crucial to develop non-visual spatial coding skills and it mostly conveys allocentric information. It has been shown that visually impaired children tend to shift from egocentric towards allocentric frames of reference later than sighted peers and that blind children are impaired in switching-perspective tasks. Moreover, research has shown that multisensory interaction of spatiotemporally coherent sensory modalities can improve perceptual spatial skills. Nonetheless, the role of sensory (visual, tactile, auditory) feedback and the influence of the size of stimulation on spatial perception during development has not been properly investigated to date. In the present studies, we aimed to investigate: (a) whether the partial and total absence of vision during growth impacts the ability to switch from egocentric towards allocentric landmarks in visual and haptic mental rotation tasks; (b) whether an increase in the size of the stimulated area produces a detrimental or beneficial effect on sensory discrimination in children with low vision. For these purposes, blind and low vision children were asked to reproduce a configuration of coins by assuming different spatial perspectives in the visual and haptic domains (a) and low vision children were asked to discriminate spatiotemporally congruent unimodal and multimodal stimulations conveyed on the arm (b). Results showed that visually impaired children had difficulties in assuming an allocentric perspective in the visual but not haptic domain (a) and in discriminating visual unimodal and multimodal stimuli (b) concerning sighted peers, independently of the age, suggesting a spatial impairment when relying on visual cues.

\section{SYMPOSIUM 13: Submission 46}

\section{Self-location in immersive virtual spaces: a developmental clinical perspective}

\section{Irene Valori, Teresa Farroni}

\section{Department of Developmental Psychology and Socialisa- tion, University of Padova, Italy}

Background: Immersive Virtual Reality (IVR) does not constitute an appropriate corollary for real-world experience, thus altering the user's sensorimotor interaction with the (virtual) space. Curiously, IVR seems to cause a somatosensory conflict between vision and proprioception (e.g. the sense of where my body is and move in space), with distinct effects on individuals depending on their developmental trajectories. While neurotypical adults show a sort of mandatory multisensory integration, children up to adolescence mainly rely on vision against the slowly developing proprioception. Moreover, neurodevelopmental conditions such as Autism Spectrum Disorders (ASD) present hypo-reliance on vision and hyper-reliance on proprioception, which are associated with motor and social difficulties. In this respect, IVR headsets have unique features to manipulate vision and proprioception and stimulate individuals' sensorimotor functioning. Aims: Our studies explored the developmental differences related to the ability of locate ourselves in space relying on vision and proprioception, in reality and within equivalent IVR simulations. Methods: We asked children and adults with typical development or ASD to sit on a swivel chair, be rotated of a certain degree and turn back to the starting position. This self-location task was performed in 2 environments (Reality; IVR) per each of three sensory conditions (Vision+Proprioception; Only-Proprioception; Only-Vision). Results and Conclusion: The ability to locate ourselves in space typically develops in the first 8 years of life and hugely relies on vision. IVR headsets can disrupt self-location accuracy, potentially improving the performance of those individuals with ASD presenting hyper-reliance on proprioception.

\section{SYMPOSIUM 13: Submission 49}

Spatial recalibration in cataract-treated individuals

Irene Senna ${ }^{1}$, Sophia Piller ${ }^{1}$, Chiara Martolini ${ }^{2}$, Monica Gori ${ }^{2}$, Elena Cocchi ${ }^{3}$, Marc Ernst ${ }^{1}$

${ }^{1}$ Ulm University, Department of Applied Cognitive Psychology, Ulm, Germany

${ }^{2}$ Unit for Visually Impaired People (U-VIP), Center for Human Technologies, Fondazione Istituto Italiano di Tecnologia, Genoa, Italy

${ }^{3}$ Istituto David Chiossone, Genoa, Italy

Since vision typically provides more precise spatial information than other sensory modalities, early visual deprivation usually results in impaired spatial cognition and mobility. Here we tested Ethiopian children and adolescents who suffered from congenital dense bilateral cataract and were surgically treated only after several years of visual deprivation. In Experiment 1, we assessed whether they could spontaneously develop an appropriate representation of the auditory space after cataract removal. We tested their ability to localize single sounds presented in a large $2 \mathrm{~d}$ space. Furthermore, we assessed their ability to understand spatial relationships among sounds by asking them to judge the relative sound position within a sequence of three spatially separated sounds. Cataract-treated individuals performed better than participants tested before surgery, and in the sound localization task they even reached the level of sighted controls. Such spontaneous recovery was mediated by visual acuity, with participants who gained higher post-surgical visual acuity exhibiting better performance in both tasks. In Experiment 2, we investigated whether an audio-visuomotor training could boost such spontaneous recalibration of spatial representations. Participants performed entertaining motor activities with the "Audio Bracelet for Blind Interaction" (ABBI), a device that associates audio-visual feedback to participants' body movements. After a short training (1-2 weeks), participants improved in most tested spatial skills and in their mobility. The present findings show that, despite years of visual impairment, cataract-treated individuals can develop more appropriate spatial representations after surgery. Such improvement can be considerably enhanced through a training strengthening the association between movements and their sensory counterpart.

\section{SYMPOSIUM 13: Submission 54}

\section{The multisensory child development and rehabilitative technology}

\section{Monica Gori}

\section{Istituto Italiano di Tecnologia, Italy}

During the first years of life, sensory modalities communicate with each other. Since 2004, we have studied how the haptic, visual, and auditory modalities interact and are integrated during development. We have observed that specific sensory modalities are crucial for developing particular skills, and the absence of one sensory input affects the development of different modalities. For example, in young children, the haptic modality is essential to perceive the size, and the lack of haptic skills (in motor impairment) affects the ability to understand the visual size of objects. Similarly, the visual information is crucial to perceive orientation, and the lack of vision (in blindness) influences haptic orientation perception and verticality perception. These results suggest a strong interaction between sensory systems during the early period of life for perceptual development. Starting from these premises, we have developed new multisensory 
technology based on multisensory feedback. The goal is to improve children's perceptual, motor, social, and learning skills when these are impaired. During the presentation, I'll present the results obtained with ABBI and TechArm. These are two wearable devices that provide audio, visual, and tactile feedbacks associated with body movement to improve haptic manipulation and social interaction. I will also show the results obtained with the WeDraw platforms, a set of applications based on audio, tactile, and visual feedback designed to improve learning skills at the elementary school level. The results are discussed, considering applications for rehabilitation settings in hospitals.

\section{SYMPOSIUM 13: Submission 120}

\section{Visual-tactile expectations and peripersonal space representations in infancy}

\author{
Giulia Orioli $^{1,2}$, Irene Parisi ${ }^{2}$, Jose Van Velzen ${ }^{2}$, \\ Andrew Bremner ${ }^{1,2}$ \\ ${ }^{1}$ School of Psychology, University of Birmingham, United \\ Kingdom \\ ${ }^{2}$ Department of Psychology, Goldsmiths, University of \\ London, \\ United Kingdom
}

Background: The influence of visual motion on the processing of bodily events offers a marker for tracing the development of human infants' perception of themselves in relation to their peripersonal space. Aims: The aim of the present research was to investigate how infants begin to link visual information about the space surrounding them with tactile information perceived on their bodies, in order to support the construction of a situated representation of their body in the surrounding environment. Methods: We presented 4- $(\mathrm{N}=20)$ and 8 -month-old $(\mathrm{N}=20)$ infants with videos of an unattended visual object moving towards or away from their body, followed by a vibrotactile stimulus on their hands. We recorded the infants' spontaneous brain activity and analysed their responses to the tactile stimuli. Results: We found that the 4-month-olds' somatosensory evoked potentials (SEPs) were enhanced in response to tactile stimuli preceded by approaching visual motion, demonstrating the early ontogeny of the cortical multisensory foundations of peripersonal space representation. Within the 8-month-olds' sample, instead, SEPs were increasingly enhanced by (unexpected) tactile stimuli following receding visual motion as age in days increased. Conclusion: These results show, for the first time, that human infants process somatosensory information differently depending on temporally and spatially distant visual information preceding it. Altogether, these findings provide important clues to the ontogeny of human selfawareness in the first year of life, and suggest important postnatal developments in infants' expectations about interactions between the body and the external world, in particular within peripersonal space.

\section{SYMPOSIUM 14: General abstract}

\section{On the Modelling of Landmarks}

Convenors: Eva Nuhn \& Sabine Timpf

\section{Geoinformatics Group Augsburg, Germany}

The use of landmarks for navigation and wayfinding has been established as a fact by re searches in spatial cognition and psychology (Lynch 1960; Presson \& Montello 1988; Sor rows \& Hirtle
1999). However, how to identify good landmarks still remains a challenge. One reason is that everything standing out from a scene might be used as a landmark (Presson \& Montello 1988). Numerous researchers examined the properties of landmarks and agreed upon socalled landmark dimensions-visual, semantic, and structural dimensions-explaining the salience of an object (Presson \& Montello 1988; Sorrows \& Hirtle 1999). While landmark identification models based on these dimensions exist (Richter \& Winter 2014), they do not appear sufficient to ensure the identification of landmarks which would also be selected by humans (Nuhn 2020). Besides the static landmark dimensions, which are dependent on an object itself, there are so called personal dimensions changing with each individual. They include dimensions such as personal interests, personal background, or prior spatial knowledge which influence the memory for salient landmarks (Albrecht \& von Stülpnagel 2018) and support traveller's spatial orientation (Schwering et al. 2017). However, the first modelling approaches incorporating personal dimensions do not identify significantly more landmarks selected by humans than a nonpersonalised model (Nuhn 2020). It seems that the interplay between different dimensions does not seem to be as simple as sometimes assumed-rather, some dimensions seem to be a precondition for the salience of a landmark (Hamburger 2020).

\section{SYMPOSIUM 14: Submission 42}

\section{Effects of landmark position and approach perspective on route retrieval}

Rebecca Albrecht ${ }^{1}$, Rul von Stülpnagel ${ }^{2}$

${ }^{1}$ University of Basel, Economic Psychology, Switzerland

${ }^{2}$ University of Freiburg, Center for Cognitive Science, Germany

Background: Object-place (or landmark) knowledge is a core element of human orientation and wayfinding. Landmarks are frequently used in route descriptions and have gained importance through the now widespread availability of personal navigation assistance systems. However, research in this area is still primarily driven by the aim to understand navigation by landmarks as an attentional process. Landmarks are distinguished by their visual, structural, and semantic salience (Sorrows \& Hirtle 1999); and visual and structural salience have been found to be a key factor to explain landmark selection for the anticipated retrieval of route directions (Röser, Krumnack \& Hamburger 2013). Aims: In contrast, there is less research on the memorability of landmarks and the ability to retrieve the turning direction associated with them. General principles of memory representations and processes and their potential interaction with findings about spatial memory are often not considered. This is surprising as this knowledge may allow for a more focused selection of landmarks which can be more promptly understood, more easily remembered and more quickly be associated with route descriptions. Methods/ Results: We present a study investigating the effects of a landmark's visual and structural salience at choice points on recall of turning directions in a VR environment. We compare memory performance for decision points presented from the same perspectives as initially studied to various altered perspectives, including the return path. Conclusion: This study extends the findings from Albrecht \& von Stuilpnagel (2018) and provides valuable insights into the stability of landmarks' mental representations during spatial operations. 


\section{SYMPOSIUM 14: Submission 43}

\section{Modelling of Landmark Dimensions}

\author{
Eva Nuhn, Sabine Timpf
}

\section{Geoinformatics Group Augsburg, Germany}

Background: The use of landmarks for navigation has been established as a fact by numerous researchers (Lynch 1960; Sorrows \& Hirtle 1999). They examined the properties of landmarks and agreed upon so-called landmark dimensions (visual, semantic, and structural, Sorrows \& Hirtle (1999)). Recently, personal dimensions such as personal interests and prior spatial knowledge were added (Nuhn 2020). Aims: The computational treatment of landmarks still remains a challenge. It seems that the existing dimensions are not sufficient to explain landmark salience (Nuhn 2020). We tackle the questions which dimensions explain an object's salience and how they are considered in landmark identification models (LIM). Methods: We revisit findings of landmark research to identify missing dimensions (landmark, personal, or other). Furthermore, we identify possible salience measures considering and extending existing salience measures with a focus on personalised landmarks. Results: The results are dimensions and their salience measures. The dimensions and salience measures can be used in LIMs to identify landmarks which would also be selected by humans. Conclusion: This study is a step towards the identification of landmarks based on different dimensions. This advances the integration of LIMs in applied navigation systems.

\section{SYMPOSIUM 14: Submission 52}

\section{Understanding landmark-based wayfinding: On the search of core factors}

\section{Kai Hamburger}

\section{Experimental Psychology and Cognitive Science Justus Liebig University Giessen, Germany}

Background: The role of landmarks in human wayfinding has been criticized lately [e.g., Montello, D. R. (2017). Landmarks are exaggerated. KI-Künstliche Intelligenz, 31(2), 193-197]. Aims: However, in order to reach a comprehensive understanding of landmark-based wayfinding, research needs to broaden its focus. Thus far, the research focus was mainly on visual, semantic, and structural aspects. Probably, structural and visual salience are investigated best, but we are still in need of systematic research on semantic salience [e.g., Hamburger, K., \& Röser, F. (2014). The role of landmark modality and familiarity in human wayfinding. Swiss Journal of Psychology, 73(4), 205-213] and other sensory modalities than just vision, for instance, auditory and olfactory information [e.g., Hamburger, K. (2020). Visual landmarks are exaggerated. A theoretical and empirical view on the meaning of landmarks in human wayfinding. KI-Künstliche Intelligenz. 34(4), 557-262]. In everyday life, wayfinding is a multimodal process. Methods: We therefore need multisensory and also broader approaches on the underlying cognitive processes. This should also include emotional aspects in human wayfinding [e.g., Balaban, C. Z., Karimpur, H., Röser, F., \& Hamburger, K. (2017). Turn left where you felt unhappy: How affect influences landmark-based wayfinding. Cognitive Processing, 18(2), 135-144]. Results and Conclusion: With this contribution, I try to highlight these aspects in order to get a step closer to a comprehensive understanding of landmark-based wayfinding and how it may be related to other cognitive strategies (e.g., serial learning, types of thinking).

\section{SYMPOSIUM 14: Submission 98}

\section{A landmark's role in spatial learning depends on its spatial extent}

\author{
Angela Schwering, Jakub Krukar
}

\section{University of Muenster, Germany}

Landmarks have been extensively investigated in spatial cognition, cognitive map making, and navigation studies. Although research reflects many aspects that differentiate landmarks, it has focussed mainly on point-like landmarks. Point-like landmarks such as points of interests, junctions, or buildings are certainly important for local orientation and may serve the role of anchor points in spatial knowledge. Global point-like landmarks may also provide orientation within a larger spatial region. Yet, landmarks can have different spatial extents. In contrast to point-like landmarks, linear and area shaped landmarks have the potential to serve as reference points connecting and structuring separate places within a combined mental representation of space. Linear landmarks such as a river, a railway, or a path can act not only as boundaries, but also as orientational features, e.g., when one is travelling along them. Area shaped features such as a park, or a neighbourhood, may act as containers and categories, helping people to hierarchically structure their knowledge about the environment. Just like point-like landmarks, linear or area shaped landmarks can be intentionally used in navigation systems to provide orientation. Sketch mapping tasks provide evidence that people use landmarks with different spatial extents to orientate themselves during navigation and to structure their spatial knowledge. We argue that linear and area shaped landmarks have strongercurrently underused - capabilities to connect distinct pieces of spatial knowledge into a consistent representation of space, and thus should be highlighted better in navigation systems.

\section{Abstracts of Talks}

\section{TALKS: Submission 5}

\section{Cognitive-motor multitasking during outdoor spatial navigation in $\mathbf{A R}$}

Federica Nenna ${ }^{1,2}$, Marco Zorzi ${ }^{1,2,3}$, Luciano Gamberini ${ }^{1,2}$

${ }^{1}$ Dipartimento di Psicologia Generale, Università degli studi di Padova, Padova, Italy

${ }^{2}$ HIT|Human Inspired Technology Centre, Padova, Italy

${ }^{3}$ IRCSS Ospedale San Camillo, Lido-Venezia, Italy

Background: When navigating the surroundings, the concurrent execution of cognitive operations (e.g., using different technological devices) increases attentional load, potentially altering external object's awareness and/or the own motor behavior. This phenomenon (cognitive-motor interference, CMI) has been usually investigated in laboratory-based settings allowing low degrees of spatial movements. Here, we availed of AR for its capacity of concurrently handling virtual and physical features to test action and cognition outdoors and in complex motion. Aims: Unlike laboratory-based walking experiments, we tested a more complex motor behavior by introducing sudden directional changes, which require a broader contextual overview and spatial orientation awareness. This was only possible through AR, which recreates hybrid experimental spaces without imposing physical constraints. We thus asked how attentional demands modulate behavioral performance of participants walking in different directions. Methods: Forty-five young participants performed a visual single-task for discriminating augmented peripheral 
targets, a navigation single-task consisting of finalized walking episodes to close augmented landmarks, and a dual-task combining the latter tasks. We evaluated the dual-tasking cost on cognitive and motor performance by analyzing reaction times, task accuracy, exploration time, and walking velocity, along with a subjective assessment of mental load. Results: A performance decrease resulted in the cognitive and motor domains under dual task compared to single tasks, which was not subjectively perceived. Conclusion: Overall, we highlighted the consequences of cognitive-motor multitasking during distracted navigation by availing of AR. AR is thus a relevant tool for creating hybrid spaces that can be leveraged for investigating cognition and action in physical motion.

\section{TALKS: Submission 7}

The empowerment effect of centesimal prismatic lenses on attention regulation

\author{
Davide Crivelli $^{1,2}$, Federico Cassioli ${ }^{1,2}$, Marco Grassi ${ }^{3}$, \\ Michela Balconi ${ }^{1,2}$
}

${ }^{1}$ International Research Center for Cognitive Applied Neuroscience-IrcCAN, Catholic University of the Sacred Heart, Milan, Italy

${ }^{2}$ Research Unit in Affective and Social Neuroscience, Department of Psychology, Catholic University of the Sacred Heart, Milan, Italy

${ }^{3}$ Family Vision Center-VTE, Sesto San Giovanni (MI), Italy

Background: The association between vision, sensorimotor integration, and attention depends on a large cortical-subcortical network. Given the interconnection of sensory and cognitive functions, an alteration of visual information flow may lead to compensation syndromes, which could hinder higher cognitive skills such attention regulation. Prismatic lenses-modulating the information exchange between the superior colliculus and the sensory nuclei of the trigeminal nerve, the cerebellum, and the frontal/parietal corticeshave been used in treating altered postural patterns, sensory integration deficit, and spatial neglect. Yet, their potential for empowerment is unexplored. Aims: This study aimed at investigating the potential of a sensory empowerment protocol supported by centesimal prismatic lenses (SiXDEVICE) with regard to attention regulation and orientation. Methods: Twenty participants were randomly divided into experimental and control groups. The experimental group completed a 4-week protocol supported by centesimal prismatic lenses. The active control group completed a visual training protocol. Attention was assessed pre-/post-training via computerized tests (Stroop, Cueing, and Flanker tasks) complemented with task-related electrophysiological (ERPs) markers of cognitive effort. Results: The experimental group showed reduced response times (RTs) at the Stroop task and a paired increase in accuracy and reduction of RTs at the Cueing task. Notably, it also showed significant post-training decrease in an ERP marker of cognitive workload (P300) along the midline. Conclusion: Present findings suggest that the use of centesimal prismatic lenses might foster attention regulation mechanisms and endogenous - over exogenous - orienting of attention, hinting at their potential as supportive tools in neurocognitive empowerment interventions.

\section{TALKS: Submission 10}

Horizontal spatial biases in time-related words: A line bisection study

Anastasia Malyshevskaya ${ }^{1}$, Federico Gallo ${ }^{1,2}$, Yury Shtyrov ${ }^{1,3}$, Andriy Myachykov ${ }^{1,4}$

${ }^{1}$ Centre for Cognition and Decision making, Institute for Cognitive Neuroscience, National Research University Higher School of Economics, Krivokolenniy Pereulok 3, Entrance 2, 101000, Moscow, Russian Federation

${ }^{2}$ Centre for Neurolinguistics and Psycholinguistics (CNPL), Vita-Salute San Raffaele University, Via Olgettina 58, 20132, Milan, Italy

${ }^{3}$ Center of Functionally Integrative Neuroscience (CFIN) Institute for Clinical Medicine Aarhus University / Aarhus University Hospital, Norrebrogade 44, bldg 1A 8000, Aarhus, Denmark

${ }^{4}$ Department of Psychology, Northumbria University, Northumberland Building, Newcastle upon Tyne, NE1 8ST, United Kingdom

Access to time words engage a complex spatial mapping where individual concepts are represented along horizontal, vertical, and sagittal axes. However, most existing studies demonstrate spatial biases in individual time units (i.e., months, days of the week, or hours of the day) using different experimental tasks and paradigms while few studies attempt to compare access to the distinct time concepts within a single study by using a uniform and comparable experimental protocol. Here, we used time units (days, months, hours) with hypothesized left (e.g., Monday) and right (e.g., Saturday) spatial biases in a horizontal line bisection study. We investigated whether horizontal time flow is perceived uniformly across different measurement units. Fifty-seven native Russian speakers (37 females, age $21.5 \pm 4.1$ years) listened to time words before using mouse cursor to indicate where that unit may be located on a horizontal line with extreme points biasing left, right, or central positioning of the processed word. Task-related reaction times (RTs) and response coordinates were recorded and analyzed using ANOVAs. Our analyses revealed (1) direction-congruent shift in participants' responses (x-coordinates, mouse endpoint) as well as (2) faster RTs in congruent conditions (e.g., left semantic bias + left position) for all time units. However, a reliable main effect of Unit Type indicated that RTs were graded in the following order: hours $<$ days $<$ months. We conclude that while left-to-right mapping of time concepts is relatively universal, the horizontal mapping is stronger for hours and days of the week, as compared to months.

\section{TALKS: Submission 14}

\section{Training-induced contraction of visual space is retinotopic, not spatiotopic} Valeria Peviani ${ }^{1}$, Andrew Haun ${ }^{2}$, Giulio Tononi ${ }^{2}$,
Lucia Melloni ${ }^{1,3}$

${ }^{1}$ Department of Neuroscience, Max Planck Institute for Empirical Aesthetics, Frankfurt am Main, Germany

${ }^{2}$ Department of Psychiatry, University of WisconsinMadison, Madison, WI, United States

${ }^{3}$ Department of Neurology, New York University School of Medicine, New York, NY, United States 
How does space feel extended and structured in the way it does? Recently, it has been argued that the perceived structure of visual space is determined by the structure and strength of lateral connections in early visual cortex. In line with this hypothesis, passivelearning training in human subjects, shown to strengthen lateral connections in animal early visual cortex, results in contraction of trained visual space, measured as the perceived distance between two locations. A key untested prediction of this account is that such experiential changes are anchored to the retinal position of the training stimulus. We administered a perceived-distance task before and after the passive-learning training in three conditions, using a between-subjects design. In the canonical condition, task and training stimuli were located in the same retinotopic and spatiotopic position; in the retinotopic condition, task stimuli were located at a different spatiotopic but the same retinotopic position as the training stimuli; in the control condition, the training was substituted with a rest period. The effect of condition on the post-training change of the perceived distance was studied by fitting a linear model. We observed a similar contraction of the post-training perceived distance in the canonical and retinotopic conditions. In contrast, in the control condition no significant change was observed. Our results thus confirm that the training results in a modulation of the experience of visual space that is anchored to the retinal position of the stimulus, strongly suggesting the involvement of early visual cortex.

\section{TALKS: Submission 15}

\section{Spatial reasoning benefits from motor imagery}

Francesco Iani ${ }^{2}$, Walter Shaeken ${ }^{2}$, Monica Bucciarelli ${ }^{1}$

${ }^{1}$ Dipartimento di Psicologia-Università di Torino, Italy

${ }^{2}$ Laboratory for Experimental Psychology, KU Leuven, Belgium

Background: According to an embodied perspective, reasoning involves sensorimotor simulations of past experiences (Barsalou 2008). Aims: We assumed that: (1) spatial reasoning relies on the construction of mental models of the states of affairs described in the premises, (2) kinematic mental models, compared to static mental models, rely on sensorimotor simulations and thus foster reasoning. Hence, instructions focusing on action should favour spatial reasoning. Methods: The participants in the experiment encountered spatial reasoning problem with valid conclusion: 4 one-model and 4 twomodel problems. Half participants were invited to imagine to move the objects while solving the problems and half participants were not. Results: We analysed participants' reaction time using linear mixed effect regression model, including Instruction (dynamic vs. static) and Model (one model vs. two models) as fixed factors of interest and Subjects and Item (i.e., reasoning problems) as crossed random effects. We detected a significant effect of Instructions ( $\beta=1498.1$, $\mathrm{SE}=735.8, \mathrm{t}=2.04, p<0.05)$ on reaction times. Both effects of Type of model $(\beta=216.1, \mathrm{SE}=531.4, \mathrm{t}=0.41)$ and interaction $(\beta=200.2$, $\mathrm{SE}=752.9, \mathrm{t}=0.27)$ were not significant. Participants, regardless the type of problems they encountered, were faster in correctly responding to problems presented with dynamic instruction $(\mathrm{M}=$ 6255.43, $\mathrm{SD}=4381)$ compared to those presented with static instruction $(\mathrm{M}=4500.35, \mathrm{SD}=1939.93)$. Conclusion: Participants were faster in drawing accurate conclusions to spatial problems when they imagined moving the objects mentioned in the premises.
TALKS: Submission 16

\section{Evidence for Cognitive Spatial Models from Ancient Roman Land-Measurement}

Andrew Riggsby

University of Texas at Austin, United States

Background: It has been influentially argued that the relative absence of cartography in Ancient Rome indicates a culture-wide failure to develop "survey"-type (multi- dimensional, allocentric) cognitive models, relying instead on (one-dimensional, egocentric) "route" models (Janni 1984, Brodersen 2003). Aims: Avoiding evidentiary controversies surrounding this past work, this paper argues that a different dataset provides clear evidence in favor of the existence of Roman survey-type cognition. Methods: The paper applies a close reading of spatial language in the Roman land-measurers' descriptions of the boundaries of irregular parcels of land (compare Taylor and Brunyé 2013; Denis 2017, chapters 10 and 11). Results: The prescriptive form of these descriptions is an itinerary defined by a series of landmarks, superficially supportive of the "route" theory. However, actual instances of such descriptions show a number of techniques that allocentrically overlay the route descriptions with survey information, including references to multiple external orientation schemes and to hypothetical other travelers, and characterization of whole spaces. The nature of these devices is not uniform across examples, but this is typical of the family resemblance that characterizes other Roman information technologies (Riggsby 2019). Conclusion: The evidence from land measurement thus undercuts the claim from the mapping argument of a lack of cognitive complexity, and it is more consistent with current understanding of the connections between different types of spatial knowledge organization (Kim \& Bock 2020).

\section{TALKS: Submission 29}

\section{Audiomotor Contributions To The Audiospatial Calibration of The Horizontal Plane}

\author{
Davide Esposito $^{1,2}$, Alice Bollini ${ }^{1}$, Monica Gori ${ }^{1}$
}

${ }^{1}$ Unit for Visually Impaired People, Istituto Italiano di Tecnologia, Italy

${ }^{2}$ DIBRIS, Università di Genova, Italy

The typical calibration of auditory cues in space is supposed to rely on visual information. However, early blind people egocentrically localize sounds in the horizontal plane as well as-or even better than-sighted ones; thus, they somehow compensate for the lack of early visual experience. The compensation may happen through coordination of auditory and motor cues according to both visual and motor experience, which is affected by early visual deprivation. This study focuses on how motor and visual experience change the contribution of audio-motor coordination to the egocentric calibration of sounds in the horizontal plane. We developed a set of steering tasks in auditory virtual reality (VR). In such tasks, participants were required to steer an arrow towards an acoustic target. Rotations of the head or trunk controlled the steering. In some conditions, the auditory consequence of head and trunk movements was altered to expose the participants' reliance on audio-motor coordination. We tested the audio-motor coordination alteration effect on the early blind, late blind, and sighted blindfolded people. We found that (1) the audiomotor coordination alteration was significantly more detrimental for early blind than late blind and sighted; (2) after the audio-motor coordination alteration, the performance was better for frontal targets 
than for lateral targets for all groups. We concluded that audio-motor coordination substitutes vision in spatial calibration only when the latter is lost early in life and that the alignment of space and body may rely on a supra-modal representation of the straight-ahead direction.

\section{TALKS: Submission 33}

\section{From action to spatial cognition: the rehabilitation program based on SaM Method}

\author{
Sara Magenes ${ }^{1,2,3}$, Laura Colautti ${ }^{1,2}$, Vania Taverna ${ }^{2}$, \\ Eleonora Carravieri ${ }^{2}$, Marina Rossi ${ }^{2}$, Annalisa Risoli ${ }^{1,2}$ \\ ${ }^{1}$ Department of Psychology, Università Cattolica del Sacro \\ Cuore-Milan, Italy \\ ${ }^{2}$ ASaM Associazione Sense and Mind-Milan, Italy \\ ${ }^{3}$ Fraternità e Amicizia Società Cooperativa Sociale \\ ONLUS_Milan, Italy
}

Background: Research from Neuroscience highlights that motor experiences underlie cognition (Gallese and Lakoff 2005) and cover a key role for intentional action and sensorimotor integration in the dynamic spatial processing (Fogassi et al. 1996). Aims: The goal is to introduce SaM Method, an innovative neuropsychological rehabilitation program, in which the embodied perspective plays a central role (Risoli 2019). Specifically, the intentional action is a fundamental element both for the processing of spaces (personal, peripersonal, extrapersonal) and for the activation of implicit and explicit learning, which support the cognitive processing of integrated abstraction levels. Methods: SaM Method operates through two types of activities, namely Basic Activities, using errorless learning techniques, and Multimodal Integration Activities, based on sensorimotor experiences affecting executive functions. They act in three different spaces: personal space (promoting mental representations concerning the body schema), peripersonal space (increasing the repertory of motor acts, the ability to carry out actions and to plan complex behaviors), extrapersonal space (contributing to plan and perform operations on mental imagery). Results: SaM Method is addressed both to subjects in developmental age and to adults. Clinical evidence supports the use of the Method with neurodevelopmental disorders, such as DCD, and traumatic brain injuries, promoting improvements in cognitive abilities, motor skills and daily activities, i.e., swimming, using phone and money (for a single case, see: Risoli et al. 2015). Conclusion: SaM Method, with a restitutive approach, is focused on enabling and improving the spatial cognition ability, starting from the body experience.

\section{TALKS: Submission 44}

\section{The practice of speleology: what is its impact on environment representation?}

\section{Veronica Muffato, Michela Zavagnin, Chiara Meneghetti \\ Department of General Psychology, University of Padova, Italy}

Background: Navigation is a complex everyday activity and can be done in different environments. Speleology involves exploring caves. It demands the ability to orient oneself using natural landmarks (e.g. shapes of rocks) and other indicators (e.g. the direction of air currents). Aims: The present study aimed to examine whether experience of navigating in this particular environment: (1) is associated with more refined visuospatial abilities; and (2) promotes the ability to form environment representations of open and underground environments.
Methods: After completing visuospatial tasks and questionnaires, 18 expert speleologists, 19 beginner speleologists, and 19 expert excursionists (as controls) listened twice to spatial descriptions, one of a path through a cave, the other of a mountain trail (in counterbalanced order). After hearing each description, participants answered true/false spatial questions and performed a map drawing task. Results: Our results showed that: (1) expert speleologists had greater mental rotation abilities and less spatial anxiety than beginners or excursionists; (2) after accounting for age, education, and individual visuospatial factors, expert speleologists answered the true/false questions better than beginner speleologists or expert excursionists-for both types of spatial description. No differences emerged between the groups in the map drawing task. Conclusion: The experience speleologists gained from exploring caves enabled them to refine their visuospatial abilities and better infer spatial relations in an environment. People's expertise in exploring environments is a factor that relates to the quality of their spatial mental representations.

\section{TALKS: Submission 45}

\section{Effect of age and space distortions on spatial body representation}

\section{Lucile Dupin}

Institut de Psychiatrie et Neurosciences de Paris, InsermUniversité de Paris, Paris

Our brain reconstructs external space in order to interpret it and interact with it. Several studies have found that the characteristics of cerebral spatial representations differ from those of the physical world. Since we must be able to interact with external space (e.g. grasp an object) based on these representations, we hypothesized that body representation could share characteristics with external space and that distortions from physical world could reflect a functional role. We assessed ( $n=60,20$ to 79 years), in absence of vision, the distortions of hand representation from the physical hand. Participants had to point (1) the perceived localization of tactile stimulations applied randomly on ten different targets (metacarpophalangeal joint and nail of each finger) and (2) the location of these targets based on verbal instructions (e.g. 'where is the nail of index finger'). We also compared hand representation to the spatial distortions of objects representation assessed by pointing the corners of a standardized imagined rectangle. We also quantified the effect of age since motor and sensory functions deteriorate with age. Our results showed that both distortions of external space representation and age affected independently the extent of hand representation. As age increased, the spatial representation of the hand decreased in size. However, the cause of this age-related shrinking of hand representation remains unidentified since we found no correlation with light touch assessment or dexterous daily activities. In conclusion, we found that distortions of body representations are influenced by independent factors including external space (e.g. objects) representation and age.

\section{TALKS: Submission 47}

\section{Aging effects on spatial switching ability} from allocentric to egocentric

\section{Renato Orti, Tina Iachini, Gennaro Ruggiero}

Laboratory of Cognitive Science and Immersive Virtual Reality, CS-IVR, Department of Psychology, University of Campania Luigi Vanvitelli, Caserta, Italy 
Background: In aging, a decline of egocentric (subject-to-object) and mainly allocentric (object-to-object) spatial representation is frequently reported. However, spatial environments in which we move are complex and processes of switching between both reference frames are necessary. Aims: Investigating the effect of aging on the ability to switch and non-switch between reference frames. Methods: The visuospatial memory performance of healthy young $(\mathrm{N}=25)$ and elderly $(\mathrm{N}=25)$ participants was compared on the Ego-Allo Switching task. Participants provided switching (from egocentric-toallocentric: Ego-Allo; from allocentric-to-egocentric: Allo-Ego) and non-switching (only egocentric: Ego-Ego; only allocentric: Allo-Allo) spatial judgments about triads of $3 \mathrm{D}$ geometrical objects. The triads were presented on plasterboard panels $(6 \mathrm{sec}$.), removed and after a delay $(5 \mathrm{sec}$.) the testing phase started in which two questions regarding the same (non-switching condition) or different (switching conditions) reference frames were given. Response time and accuracy were recorded. Results: A negative impact of aging on switching abilities emerged: elderly performed worse than younger participants in Allo-Ego switching judgments. Such negative effect of aging appeared attenuated when the first reference frame was egocentric (Ego-Allo). In the non-switching conditions, elderly participants performed worse than young adults in processing Allo-Allo judgments. Conclusion: These findings suggest that, alongside allocentric impairments, aging also compromises the ability to flexible switch from allocentric to egocentric representations. Such detrimental effects of aging may be relate to the dysregulation in the locus coeruleus-noradrenaline system of prefrontal cortex and to the concurrent deterioration of hippocampal and posteromedial areas.

\section{TALKS: Submission 51}

\section{Challenging automated landmarks-based route- guidance with field testing}

\section{Elise Grison, Céline Durupt, Archana Prabhakar, Jean-Michel Dagba, Simone Morgagni}

\section{SNCF, Innovation \& Research, France}

It has been repeatedly demonstrated that human beings use landmarks to navigate and give directions (Yesiltepe et al. 2019). Recent works of research have tried to develop solutions to integrate landmarks in models that automatically generate route-guidance instructions. In this framework, we developed and tested an indoor-outdoor, multilevel, landmarks-enriched pedestrian routing algorithm (e.g. "Go upstairs and turn right after the bakery to enter the station"). A pedestrian routing algorithm using landmarks was first developed with OpenStreetMap data based on recent findings (Rousell et al. 2017). Contrary to recent research, results of a first field study show that instructions enriched with landmarks did not improve navigation despite being positively perceived. A second experiment was thus conducted to explain this difference and to highlight eventual correlations with the interactive map specifications. A new set of participants were asked to navigate routes using (1) a non-interactive map without instructions, (2) manually proposed instructions without a map, and three other variants consisting of a map with (3) basic instructions, (4) instructions enriched with landmarks and (5) manually improved instructions enriched with landmarks. Results showed that without manual adjustments, the algorithm created ambiguities that did not improve passengers' orientation. The manually improved version, however, led to better performances even without a map. This work highlights that the automation of route-guidance using landmarks is feasible, but that user incomprehension will require additional research and significant enhancements. Finally, field studies renewed questions regarding the integration of landmarks in route-guidance systems and provided new cues for improvement.

\section{TALKS: Submission 53}

\section{Temporal perception: how does multisensory} integration occur?

\author{
Giovanni Cantarella ${ }^{1}$, Patrizia Silvia Bisiacchi ${ }^{1,2}$ \\ ${ }^{1}$ Department of General Psychology, Università di Padova, \\ Italy \\ ${ }^{2}$ Padova Neuroscience Center, Italy
}

Background: A number of investigators have argued that the brain regions shared by space and time might play a similar process in the two domains. Literature revealed that different sensory modalities are not equivalently suited for the perception of a given event. Nevertheless, the ways sensorial channels co-interact among each other to achieve a stable representation of temporal information is still a matter of debate. Aims: When considering sighted children, the presence of a potential interference in multisensory conditions led to a trade-off between two senses (sight and hearing). To what extent could a condition of sensory deprivation (both auditory and visual) affect time estimation performances of young healthy adults? Methods: Fifty-six participants performed a time bisection task. The presence or absence of sensorial deprivation, considering two sensory (visual and auditory) modalities was manipulated. A within-subjects design was set up. For each participant, the order of administration was randomized between modalities. Repeated-measures ANOVAs were conducted. The emerging significant interactions were followed by post hoc analyses with Bonferroni's correction. Results: Within the auditory modality, subjects did not show statistically significant differences in temporal performances. In contrast, a significant interaction was found within visual modality among the presence and absence of auditory deprivation. Conclusion: These results can be interpreted as evidence of an optimal multisensory (audio-visual) integration of the auditory modality that leads to increased sensitivity to time. The demonstration of a higher variability in visual temporal estimation performances shows further investigation of this sensory modality for time perception is required.

\section{TALKS: Submission 58}

\section{Motor Simulation in Sentence-Picture Verification: Beyond Ostarek et al. (2019)}

\author{
Alex Miklashevsky, Martin H. Fischer
}

\section{Potsdam Embodied Cognition Group, University of Pots- dam, Germany}

Background and Aims: Ostarek et al. (2019) claimed a conclusive demonstration that language comprehension relies profoundly on visual simulations. They presented participants with visual noise during sentence-picture verification (SPV) and measured lateralized button response speed. The authors selectively eliminated the classical congruency effect (faster yes decisions when pictures match the objects implied by the sentences) with "high level" noise made from images of other objects. However, that visual noise included tool pictures, known to activate lateralized motor affordances. Moreover, some of their sentences described motor actions. This raises the question whether motor simulation may have contaminated their results. Methods: Replicating Ostarek et al. (2019), 33 right-handed participants performed SPV but either without visual noise or while 
viewing (a) only left-handled or (b) only right-handled or (c) alternatingly left- and right-handled tools. Accuracy and reaction times of manual yes responses were analyzed. Additionally, hand-relatedness of sentences was rated. Results: Replicating Ostarek et al. (2019), the classical SPV congruency effect appeared without noise and vanished when alternatingly handled tools were presented. Crucially, it re-appeared when noise objects were consistently either left- or righthandled. Higher hand-relatedness of sentence content reduced SPV performance and accuracy was lower with right-handled noise. Conclusion: First, we demonstrated an interaction between motorrelated language, visual affordances and motor responses in SPV. This result supports the embodied view of language processing. Second, we identified a motor process not previously known in SPV. This extends our understanding of mental simulation and calls for methodological controls in future studies.

\section{TALKS: Submission 59}

\section{City exploration through the eyes of the visually impaired}

\author{
Selin Kocabiyik ${ }^{1}$, Didem Kan-Kilic ${ }^{2}$ \\ ${ }^{1}$ Izmir University of Economics_Design Studies Master \\ Programme, Turkey \\ ${ }^{2}$ Izmir University of Economics_Faculty of Fine Arts and \\ Design_Interior Architecture and Environmental Design \\ Department, Turkey
}

Is urban space something seen, experienced and perceived only with eyes? If we remove the sense of sight from this experience, will this experience be incomplete? Based on these questions, we will find the answer to what an urban experience would be like without the sense of sight by exploring the city together with the congenital visually impaired participant. The main hypothesis is to reveal and embody tactical spaces, creative productions and ideas of visually impaired individual in everyday life and show this creative production/relationship that the visually impaired establishes with the urban space. We try to understand the tactics developed by the visually impaired to reveal the other dimensions and codes of the city while navigating seven different walking routes determined by the researcher in the city (İzmir/Turkey). The visually impaired participant, who received photography training for 1 month before the study, walks each of these routes with the researcher on the specified days. During this photowalk, the visually impaired participant is expected to verbally describe the experience. The photograph has been chosen as a tool of communication with the sighted and visually impaired. Among the expected results of the study, it will be shown how tactical ideas and productions in daily life are applied to the urban space and how they transform the urban space. By looking at the tactics formed by the visually impaired individual against the strategies, it can be determined that these are productions that shape and transform the urban place in a spatial sense.

\section{TALKS: Submission 61}

Paradigm constraints on moral decision space: a model fit to mouse-tracking data

Flora Gautheron ${ }^{1,2}$, Jean-Charles Quinton ${ }^{2}$, Annique Smeding ${ }^{l}$

${ }^{1}$ Univ. Grenoble Alpes, Univ. Savoie Mont Blanc, Chambéry, France

${ }^{2}$ Univ. Grenoble Alpes, CNRS, Grenoble, France
Background: A multitude of factors may influence moral decisions, leading to complex dynamics that call for nuance. Yet, experimental paradigms are often restricted to two-alternative forced choice tasks, with alternatives placed in opposite corners of the screen space. Aims: Could paradigm response mode (two-alternatives or continuous scale) influence how morality is cognitively represented and processed, either relying on continuums or categories? Methods: We built a computational model of (moral) decision-making based on differential equations (dynamic neural fields coupled with sensorimotor control, extending classical drift diffusion models) in which a 1D population of neuronal units maps a moral judgment scale (discrete or continuous). Neural fields usually operate on continuous spaces (e.g., sensorimotor), but allow the emergence of spatially localized attractors. Spatiotemporally coherent activity across the neural field reflects convergence in the decision space, while generating (mouse) trajectories aiming at on-screen response locations. Results: Simulated data were fitted to mouse-tracking data previously collected on human participants, where the dynamics of participants' judgments on moral statements was recorded using the computer mouse. Based on paradigmatic constraints implemented in the model, it successfully produced adequate mouse trajectories in both binary and continuous response modes, possibly reflecting how the spatial representation of responses impact decision-making. Conclusion: Adjusting parameters in our model based on empirical data allowed us to bridge the gap between two-alternative forced choice and continuous scale paradigms, possibly giving insights into processes underlying human decision-making, and whether moral decision dynamics would differ depending on response mode.

\section{TALKS: Submission 62}

\section{The role of spatio-temporal components in egocentric and allocentric encoding}

\author{
Filomena Leonela Sbordone, Gennaro Ruggiero, \\ Francesco Ruotolo, Tina Iachini
}

Laboratory of Cognitive Science and Immersive Virtual Reality, CS-IVR, Department of Psychology, University of Campania Luigi Vanvitelli, Caserta, Italy

Background: The ability to represent everyday events requires the integration of spatial and temporal information. Studies about spatial memory have mainly used static layouts and have neglected the relationship between spatial and temporal components in spatial encoding according to frames of reference (FoRs). Aims: We explore the relationship between temporal information and spatial information based on egocentric (subject-to-object) and allocentric (object-toobject) FoRs. Methods: Thirty-six participants had to memorize as accurately as possible two stimuli (geometric 3D objects) that were presented one after another (400 msec each) on a virtual panel with an allocentric stable marker (a black bar). Participants judged what stimulus appeared closest to them (egocentric condition) or to the bar (allocentric condition). In both conditions, participants also judged which stimulus appeared first. Results: Egocentric judgments were more accurate when the object closest to the body appeared first than second, suggesting a primacy effect. In contrast, allocentric judgments were more accurate when the object closest to the bar appeared second than first, suggesting a recency effect. Moreover, participants performed better with temporal than spatial judgments. Conclusion: The opposite effect of temporal order on FoRs suggests that temporal mechanisms might have a specific and differential effect on spatial encoding. Furthermore, the body-based anchoring of egocentric encoding leads to the representation of an episodic event that links what appears first with what appears closest to the body; as for 
allocentric encoding, facilitation for the target that appeared second would suggest a different role in memory systems to be investigated in future studies.

\section{TALKS: Submission 68}

\section{Adults' spatial scaling across different perceptual conditions}

\author{
Magdalena Szubielska ${ }^{1}$, Marta Szewczyk ${ }^{1}$, \\ Wenke Möhring ${ }^{2,3}$ \\ ${ }^{1}$ Institute of Psychology, The John Paul II Catholic \\ University of Lublin, Poland \\ ${ }^{2}$ Faculty of Psychology, University of Basel, Switzerland \\ ${ }^{3}$ Martin-Luther University Halle-Wittenberg, Germany
}

The present study examined differences in adults' spatial-scaling abilities across three perceptual conditions: (1) visual, (2) tactile, and (3) visual and tactile. Participants $(\mathrm{N}=60)$ were instructed to encode the position of a convex target presented in a simple map without time limit. Immediately after encoding the map, participants were presented with a referent space and asked to place a round disc at the same location from memory. Spaces were designed as tactile graphics. The position of targets varied along the horizontal dimension. The referent space was constant in size while sizes of maps were systematically varied, resulting in three scaling factor conditions: $1: 4$, $1: 2,1: 1$. One-third of participants was blindfolded throughout the entire experiment (tactile perceptual condition). The second group of participants was allowed to see the graphics (visual condition); the third group was instructed to see and touch the graphics (visual and tactile condition). Participants produced larger errors in the tactile condition as opposed to the two visual conditions. There was also a significant interaction effect between scaling factor and perceptual condition. Participants in both visual conditions showed a linear increase in errors with higher scaling factors whereas in the tactile condition, this relation was quadratic. Findings imply that adults' spatial-scaling performance across different perceptual domains seems differently affected by scaling factors, which may refer to the usage of different strategies. Results may be useful for adjusting training for map reading depending on the availability of visual information.

\section{TALKS: Submission 69}

\section{Peripersonal Space in Virtual Reality}

\section{Lauren Buck, Bobby Bodenheimer}

\section{Vanderbilt University, United States}

Background: 3D immersive virtual environments (IVEs) allow users to interact with the space around them, near and far, in a way that is similar to the real world. The near space close to our bodies that we define as our reaching and grasping distance (peripersonal space) is plastic - it expands and contracts based on the context of every interaction. To create high-quality virtual reality experiences, peripersonal space should expand and contract as it does in the real world. Aims: In this talk, we address how we measure peripersonal space in IVEs and what factors of IVEs allow it to be mediated naturally. These factors include interactions with objects, agents, and those of self-avatars such as bodily dimensions and perceived embodiment. Methods: Over the course of several experiments using a novel methodology developed by Serino et al. (2018), responses to tactile stimuli were recorded and peripersonal space boundaries were determined in IVEs while contextual conditions were varied. Results: Our results show that peripersonal space boundaries can be delimited in IVEs, and that they are responsive to different interaction contexts. Peripersonal space boundaries modulate based on varying characteristics of objects and agents and are affected by embodiment. However, when bodily dimensions of self-avatars are subtly but noticeably manipulated, peripersonal space boundaries may not change. Conclusion: While peripersonal space boundaries in IVEs are modulated in ways similar to how they are in the real world, characteristics of IVEs affect these boundaries and must be taken into account.

\section{TALKS: Submission 70}

\section{Individual Differences in Navigating a Threatening Virtual Environment}

\author{
Meredith Minear, Tesalee Sensibaugh
}

\section{University of Wyoming, United States}

Background: Individual differences in temperament related to approach and avoidance motivation have been argued to make a large contribution to personality and behavior. Behavioral inhibition system (BIS) sensitivity may influence how well individuals are able to effectively use certain navigation strategies within anxiety-provoking situations. Aims Our study investigated the relationship between individual differences in (BIS) sensitivity - and allocentric (cognitive map-based) and egocentric (stimulus-response-based) navigation ability under conditions of environmental threat. Methods: A total of 214 participants completed a virtual navigation task (a Morris water maze) within an anxiety provoking immersive virtual environment consisting of a shark-infested ocean presented through the HTC Vive Pro. Half of the participants learned the location of the platform relative to the configuration of multiple large, faraway ("distal") landmarks and the other half learned the location of the platform relative to the position of nearby ("proximal") landmarks consisting of various small buoys. Movement within the virtual environment was controlled using an omnidirectional treadmill. Participants also completed multiple cognitive and personality measures. Results: Our analysis found that participants' levels of behavioral inhibition were related to allocentric navigation performance, but this was moderated by participants' spatial ability with higher behavioral inhibition predicting worse performance among individuals with low but not high levels of spatial ability. By contrast, behavioral inhibition was not related to egocentric navigation performance. Conclusion: Our data are consistent with other work suggesting that personality traits related to cautiousness and anxiety may predict the use of relatively inefficient navigation- and exploration-related strategies, and poorer spatial navigation ability under threat.

\section{TALKS: Submission 72}

\section{On temporal versus spatial scale}

\section{Hans Skov-Petersen}

\section{Dept. of Geocience and NAtural Ressource Management, University of Copenhagen, Denmark}

Background: Time is everywhere. In our daily lives and in the way, we mediate ourselves and our environment. In geography, and other spatial sciences, most implicitly relates to time. In digital geodata and GIS, time is rarely, explicitly handled-conceptually and during recording, storage, and analysis of data. Time can be conceptualized, 
perceived and recorded at different scales. The spatial scales embedded as metadata, reflects the conception of the phenomena and is often seen as a token of its spatial quality, granularity, and trustworthiness, considering a given use case. The presentation will propose a similar approach to time and temporal scales. Aims: With reference to spatial concepts, this presentation will propose a conceptual basis of time and temporal scale, and will accordingly suggest a framework for handling of temporal scale in relation to geo-data and spatial/temporal quality, representation, and analysis. Methods: By a structured analysis of spatial scale and phenomena, as conceived and represented in GIS, a notion of temporal scale will be developed. One difference between the concepts of temporal and spatial scale is that spatial scales relate objective/physical/corporal measures with it representation on paper on the screen, whereas 'measures' of time in our minds are less objective. Accordingly, notions of temporal scale require additional considerations. Results: The presentation will suggest an approach to notion of temporal scale that will enable application of temporal scale as metadata to geodata. Conclusion: We need to close the 'temporal gab' in the way time is handled scale in present-day geodata.

\section{TALKS: Submission 76}

\section{Idiosyncratic benefits in multisensory spatial navigation}

Silvia Zanchi, ${ }^{l, 2}$, Luigi F. Cuturi ${ }^{l}$, Giulio Sandini ${ }^{l}$, Monica Gori ${ }^{1}$

\section{${ }^{1}$ Italian Institute of Technology, Italy \\ ${ }^{2}$ University of Genoa, Italy}

Background: When moving through space, we encode multiple sensory cues that guide our orientation through the environment. Integration between visual and self-motion (e.g., vestibular/proprioceptive) cues is known to improve navigation. However, spatial navigation may also benefit from multisensory external signals, such as auditory and visual. Aims: The present study aimed to investigate how humans exploit combined auditory and visual landmarks to improve their navigation abilities. Methods: Healthy subjects participated in the study. Two experiments with different cue reliability were conducted. In both, participants' task was to return an object to its original location by using landmarks, which could be visual-only, auditory-only, or audiovisual. The testing room was darkened to reduce unintended sensory inputs. We ran a conflict condition with spatial discrepancy between visual and auditory landmarks to assess each cue's weight. We took error and variability of object relocation distance as measures of accuracy and precision. Results: We observed comparable accuracy and precision in both experiments when spatially navigating with visual-only and audiovisual landmarks but greater error and variability with auditory-only landmarks. Splitting participants into two groups based on given unimodal weights revealed that only subjects who associated similar weights to auditory and visual cues showed reduced variability in audiovisual conditions, meaning greater precision. Conclusion: These results suggest that optimal multisensory integration occurs depending on idiosyncratic cue weighting. This finding indicates that functional development of multisensory procedures to aid orientation and mobility must consider individual differences in the ability to encode external landmarks.

\section{TALKS: Submission 84}

\section{Mental Spaces in Random Number Generation}

\section{Haiming Yang}

\section{Peking University School of Public Health, China}

Inhibition of return (IOR) refers originally to the field of spatial attention. However, it has also been demonstrated that humans have a tendency of repetition avoidance in random number generation (RNG). One question is whether temporal factors influence repetition avoidance in RNG. Participants were instructed to orally generate a sequence of numbers at a defined pace $(0.5 \mathrm{~s}, 1.5 \mathrm{~s}, 3 \mathrm{~s}$ or $4 \mathrm{~s} /$ response) from a response pool (i.e. numbers $0-5$ and $0-9$ ). For each response, all alternatives should have the same chosen probability, and responses should be independent from each other. Human generated random sequences differed drastically from computer-simulated pseudo-random sequences. The frequency distribution of the repetition gap (referring to how many numbers are between two identical numbers in the generated sequences) showed a stable "three-component" characteristics: an IOR-like inhibition at small distances, followed by a favored cycle period "peak frequency "and an exponential decay component. The robust IOR-like repetition avoidance demonstrates that humans tend to avoid repeating the same number at small distances even though they try to generate independent responses. The existence of the "peak frequency" indicates an endogenous oscillatory process of returning to the same item. Furthermore, repetition is affected by the number of items in the response pool, suggesting an effect casted by mental space. The time interval between responses only has a minor effect. Thus, an endogenous oscillatory process in random number generation is item-based rather than time-based defining a specific mental space.

\section{TALKS: Submission 87}

\section{The anatomical pathways underlying spatial versus non-spatial attention}

\author{
Marco Bedini ${ }^{1}$,Emanuele Olivetti ${ }^{1,2}$, Paolo Avesani ${ }^{1,2}$, \\ Daniel Baldauf $f^{l}$ \\ ${ }^{1}$ Center for Mind/Brain Sciences (CIMeC), University of \\ Trento, Italy \\ ${ }^{2}$ NeuroInformatics Laboratory (NILab), Bruno Kessler \\ Foundation (FBK), Trento, Italy
}

Background: The frontal eye field (FEF) and the inferior frontal junction (IFJ) are crucially involved in visual attention, working memory, and cognitive control. Neuroimaging evidence, however, suggests that the role of FEF and IFJ in these functions can be dissociated according to their selectivity to spatial versus non-spatial information, respectively. Aims: We propose that the divergence in the connectivity fingerprints of FEF and IFJ underlies their specialized roles in guiding visual selection. We hypothesize predominant structural connectivity of FEF with the dorsal visual pathway compared to IFJ, and vice versa, predominant structural connectivity of IFJ with the ventral visual pathway compared to FEF. Methods: We first performed an activation likelihood estimation fMRI meta-analysis to accurately infer the localization of FEF and IFJ in MNI152 space. We only included functional localizers for FEF, and covert attention, working memory, and cognitive control paradigms for IFJ (both $\mathrm{N}=20$ ). We then employed the resulting peak coordinates to perform the seed-based analysis of publicly available diffusion MRI data using probabilistic tractography. Results: We show that in the right hemisphere, the connectivity likelihood of FEF with the superior parietal lobule is higher 
than IFJ, whereas the connectivity likelihood of IFJ with the lateral temporal cortex is higher than FEF. Conclusion: We report for the first time evidence of segregated anatomical pathways that could underlie the control of spatial versus non-spatial attention from FEF and IFJ, respectively. These results may open up new possibilities of fractionating the attention networks based on the representational content encoded.

\section{TALKS: Submission 89}

\section{Attention-based Integration of Visual Context in Place Representations}

\section{Cagatay Soyer}

\section{Independent researcher, currently not affiliated}

Animals use sensory signals and memory to build internal representations of space. Recently, it has been shown that such representations extend beyond the geometry of an environment and also encode rich sensory experiences usually referred to as 'contextual' or 'sensory' inputs. In mammals, these inputs appear to be converging on the hippocampus as a key area for spatial representations and memory. How geometric and external sensory inputs (such as visual context) are combined into a coherent place representation is not fully understood. Here, I propose an abstract model of an attention-based spatial representation integrating visual features and geometric information obtained from path integration and other measurements. Inspired from earlier work on place representation and attention-based recognition in mobile robots, a key element of the model is the accumulation and registration of attentive visual features via successive fixations on the interesting parts of an environment. Eye and head movements can be directed by bottom-up saliency, as well as top-down control, potentially contributing to the stability of spatial representations. Together with the selection and contribution of attentive features (i.e. non-spatial attention), these mechanisms encode the animal's goal or specific interest in the place together with its position and orientation of relevant features. I show that a place representation network featuring such a model can explain the complex responses of hippocampal place cells. With further development, the model has the potential to generate new hypotheses for some of the less understood properties of spatial representations, such as context-based recall and place cell remapping.

\section{TALKS: Submission 92}

\section{Investigating embodied simulation experience in perception of architectural space}

\author{
Elif Aktaş Yanaşs ${ }^{1,2}$, Leman Figen Gül ${ }^{2}$ \\ ${ }^{1} \ddot{O}$ zyegin University, Turkey \\ ${ }^{2}$ Istanbul Technical University, Turkey
}

Architecture as an embodied and multimodal experience has been the topic of recent studies inspired by the conceptual approach of late nineteenth-century's empathy theories and related experimental research on embodied simulation theory. Experimental studies aimed at understanding the embodied experience in architectural space perception are mostly addressed in a framework where neuroscientific methods are centered. This study aimed to set up a framework specific to architectural design research to investigate embodied simulation experience in perception of architectural space. Thus, it provides a new perspective in the light of neuroscience and design cognition studies for exploring embodied experience in architecture. With this regard, an experimental setting has been developed in which quantitative and qualitative techniques are blended, adapting from both design research and neuroscientific research. The experimental setting consists of three stages; consideration of architectural photographs to be shown to participants, designing of survey questions to collect subjective reports of the participant and determining of empathy scores by The Empathy test. The experimental setting has been tested by 21 architectural and interior design students who have participated voluntarily in the research. This paper presents the structural framework of the experimental settings and the preliminary results of the research which are created in order to understand the embodied simulation experience in perception of architectural space.

\section{TALKS: Submission 96}

Using Mobile Augmented Reality for Spatial Cognition

Soumyajit Chakraborty ${ }^{1}$, Yu Zhao ${ }^{1}$, Jeanine Stefanucci ${ }^{2}$, Sarah Creem-Regehr ${ }^{2}$, Bobby Bodenheimer ${ }^{1}$

${ }^{1}$ Department of Electrical Engineering and Computer Science,

Vanderbilt University, United States

${ }^{2}$ Department of Psychology, The University of Utah, United States

Background: With augmented reality (AR), people interact with virtual content displayed within the real environment. AR cues and objects can be used to conduct rigorously controlled experiments to understand the mechanisms underlying human perception of the environment. Prior work has investigated whether the addition of AR cues to the real environment can improve people's spatial perception when using such devices. Little research has assessed people's perceptions of the environment when virtual objects are presented using a mobile AR display, such as a smartphone or tablet. Researchers can now deploy AR apps that allow them to conduct controlled studies "in the wild." Aims: In the context of two experiments, we discuss the challenges and limitations of mobile AR for studying spatial perception. Methods: Experiment 1 tested people's abilities to judge whether they could pass through a virtual aperture or step over a virtual gap, an affordance judgment study. Experiment 2 evaluated how social cues affected egocentric distance judgments of a standing or coughing, masked or non-masked virtual human avatar, a distance estimation study. Results: Results show people underestimated their capabilities for both actions, but accuracy increased after training with AR cues in Experiment 1. In Experiment 2, distance estimates were accurate with no effects of mask or coughing. Conclusion: These studies suggest that mobile AR displays can be used to reliably assess spatial perception. Widespread adoption of these apps for research on spatial perception presents an opportunity for researchers going forward.

\section{TALKS: Submission 100}

\section{Simultaneous Locomotion and Interaction in VR:} Walking $>$ Leaning $>$ Controller

Bernhard E. Riecke ${ }^{1}$, Abraham M. Hashemian ${ }^{1}$, Ashu Adhikari ${ }^{1}$, Ivan Aguilar ${ }^{1}$, Ernst Kruijff, ${ }^{2,}$, Markus von der Heyde $e^{3,1}$

${ }^{1}$ Simon Fraser University, School of Interactive Arts and Technology, Vancouver, BC, Canada

${ }^{2}$ Institute of Visual Computing, Bonn-Rhein-Sieg University of Applied Sciences, Germany

${ }^{3} v d H-I T$, Germany 
Locomotion and 3D interaction are both critical for many Virtual Reality applications. However, prior research typically studied them in isolation, and there is surprisingly little research investigating interaction while moving - even though we can readily do that when physically walking. To address this gap, we designed a dual-task experimental paradigm (inspired by BeatSaber), which combines a maneuvering task (staying inside and rotating with a continuously moving virtual enclosure) with an interaction task (using the LightSaber to continuously point towards ("stab") the center of upwards moving balloons appearing in $1 \mathrm{sec}$ intervals). We compared four locomotion conditions of decreasing amount of embodiment and proprioceptive/vestibular self-motion cues: Physical walking; handsfree standing leaning-based (Naviboard) and seated leaning-based (HeadJoystick) interface; and handheld controller (thumbstick-velocity-control with controller-directed steering using their nonprimary hand). Participants wore an HMD, used their primary hand for interaction, and physically rotated for all interfaces. As predicted, walking showed the highest performance in terms of locomotion score, interaction score, and combined dual-task score, whereas the handheld controller performed worst. Using leaning did, however, show significant performance improvements over controller-based locomotion, even though participants always physically rotated. This performance benefit was more pronounced for the (arguable more embodied) standing leaning-based interface (NaviBoard) compared to the seated interface (HeadJoystick). Together, this suggests that more embodied leaning-based interfaces that provide at least minimal proprioceptive/vestibular translation cues in the direction of virtual self-motion can help to get us one step closer to the ease of natural walking when physical locomotion is unfeasible.

\section{TALKS: Submission 106}

\section{Spatial skills mediate cognitive efficiency and fitness-to- drive}

\section{Luigi Tinella, Antonella Lopez, Alessandro Oronzo Caffo, Andrea Bosco}

Department of Educational Science, Psychology, Communication, University of Studies of Bari, Italy

Background: Visuospatial skills are considered the main factors among the cognitive predictors of real-world driving. In particular, both the object- and self-based spatial mental transformations, often measured through the mental rotation and perspective taking test respectively, have been recently linked to measures of fitness-to-drive in young and adult drivers. Evidences on older drivers are scarce. Aims: The present study was aimed to investigate the relationships between global cognitive functioning, spatial mental transformation skills, and cognitive prerequisites for the fitness-to-drive during driver's lifetime. In particular, the study sought to investigate the following hypothesis: the relationship between cognitive functioning and driving prerequisites is mediated by spatial mental transformation skills. Methods: One hundred and eighty-three active drivers, aged from 18 to 91 were enrolled in the study. Participants completed the Montreal Cognitive Assessment, the Mental Rotation Test, the Perspective Taking-Test, and a computerized driving task measuring for Resilience of Attention, Reaction Times and Perceptual Speed. Results: The negative effect of age was found on all the prerequisites for the fitness-to-drive. Measures of mental rotation and perspective taking were found to mediate differentially the effect of cognitive functioning on Resilience of Attention and Perceptual Speed. Conclusion: The results suggested that general cognitive efficiency is linked to spatial mental transformation skills and, in turn, to drivingrelated cognitive tasks contributing to safety driving in the lifespan.

\section{TALKS: Submission 107}

\section{Left spatial neglect encompasses impaired verticality perception}

\author{
Rémi Lafitte, Marie Jeager, Céline Piscicelli, Eve \\ Dupierrix, Shenhao Dai, Anne Chrispin, Patrice Davoine, \\ Dominic Pérennou
}

Laboratoire de psychologie et neurocognition CNRS UMR 5105, Grenoble-Alpes université, clinique de médecine physique et réadaptation, CHU de Grenoble-Alpes, 38034 Grenoble, France

Backgrounds. There is a mounting evidence that, after stroke, impaired internal model of verticality is strongly related to spatial neglect (SN). It raises questions as to which modalities of $\mathrm{SN}$ and verticality perception are related to each other? We assume that this link stems from a strong relationship between body neglect $(\mathrm{BN})$ and postural vertical (PV), both referring to the body. Aims: To analyse relationships between $\mathrm{BN}$, non-body neglect (NBN), PV, and visual vertical $(\mathrm{VV})$, in a series of individuals with right hemisphere stroke (RHS). Methods: We investigated a series of 77 individuals with RHS, with complete assessments of BN and NBN (four tests each), $\mathrm{VV}$ and $\mathrm{PV}$, performed at the same time between one and three months post-stroke. Abnormality was diagnosed from two pathological tests both for SN and verticality perception. Composite scores were computed for BN and NBN. Results: All individuals (Median age $=67)$ with verticality perception impairment $(\mathrm{N}=26)$ had $\mathrm{SN}(\mathrm{N}$ $=52$ ), but the reverse was not true. Whatever the modality, SN and verticality biases were moderately correlated ( $r$ ranging from -.64 to -.41 ; all $p<.001$ ). A principal component analysis revealed that data variance was mainly explained by one component, comprising all factors with the following loadings: $\mathrm{BN}=.88, \mathrm{NBN}=.84, \mathrm{PV}=-.80$, $\mathrm{VV}=-.73$. Conclusion: Our results reveal that $\mathrm{SN}$ encompasses impaired transmodal verticality perception, suggesting that biased verticality perception is a form of graviceptive neglect. BN, NBN, $\mathrm{PV}$, and VV belong to the same dimension. The attentional component of SN likely explains their moderate correlations.

\section{TALKS: Submission 110}

\section{What are the determinants of tilted writing and drawing after stroke?}

Rémi Lafitte, Flora Diaine, Olivier Carré, Shenhao Dai, Anaï Verdier, Céline Piscicelli, Dominic Pérennou

Laboratoire de psychologie et neurocognition CNRS UMR 5105, Grenoble-Alpes université, clinique de médecine physique et réadaptation, CHU de Grenoble-Alpes, 38034 Grenoble, France

Background: Spatial dysgraphia after a right hemispheric stroke (RHS) is usually considered a sign of spatial neglect (SN). A recent case study suggested that counterclockwise writing tilt is rather due to a biased verticality representation. Aims: To test, in a series of patients, the hypothesis that counterclockwise writing and drawing tilt stems from a biased verticality representation. Methods: We tested 130 individuals, 69 consecutively tested after a RHS (1-3 months), and 61 matched healthy controls (age, gender, education). The orientations of their writing (lines, margin) and drawing (baselines of the Gainotti scene) were measured by the lines and baselines' horizontal tilts and the margin's vertical tilt (companion abstract). Normality was defined as mean \pm 2 SD from controls' data. Perceptions and 
actions with respect to gravity were investigated with the postural vertical (PV), the visual vertical (VV), and a lateropulsion scale (SCP). SN was quantified with a composite score (0-100) obtained from eigh tests of body and non-body neglect. Results: With respect to cutoffs (lines $=-2.9^{\circ}$; baselines $=-1.3^{\circ} ;$ margin $=\mathrm{c} 4.6^{\circ}$ ), 22 patients $(32 \%)$ showed at least one abnormal criterion. They differed from others (all $p<.001$ ) by greater biased verticality representation $\left(\mathrm{PV}=-6.1^{\circ}\right.$ vs. $-1.7^{\circ} ; \mathrm{SCP}=1.9^{\circ}$ vs. $\left.0.5^{\circ}\right)$ and $\mathrm{SN}(23.2$ vs. 10.7$)$. Based on step-wise logistic regression models, PV tilt was the primary determinant of abnormal writing and drawing tilt $(p<.01)$, whereas SN had only marginal influence $(p=.06)$. Conclusion: Our data reveal that tilted writing or drawing is frequent after RHS, mainly related to a tilted internal model of verticality.

\section{TALKS: Submission 113}

\section{Duration perception in portraits with different perspectives}

Youqing Zhou, Yan Bao

School of Psychological and Cognitive Sciences, Peking University, China

Preference for depicting left-front perspective faces in western portraits has been well noticed and discussed. In a recent study, it has been suggested that viewers have higher implicit perceptual saliency of the left-front perspective portraits as compared the right-front perspective ones (Wang \& Bao 2020). Given the asymmetrical feature of perceptual processing of portraits, it remains an open question whether the subjective durations of left-front and right-front perspective portraits with same physical presentation time are also different. The present study aimed to answer this question with two different temporal paradigms. One is temporal reproduction in which subjects are asked to reproduce the duration of a previously presented stimulus. The other is temporal bisection paradigm in which subjects are required to compare the temporal stimulus to two reference stimuli (i.e., "long" and "short") held in memory. Different durations are manipulated for both left-front perspective and right-front perspective portraits and their corresponding mirror reversals. Preliminary results from a group of university students will be presented and the different sensitivity in perceiving asymmetrical portraits of faces as well as their influence on duration perception of these portraits will be discussed.

\section{TALKS: Submission 114}

\section{Sequential effect in duration reproduction is from both perception and response}

\author{
Tianhe Wang ${ }^{1,2}$, Yingrui Luo ${ }^{1}$, Yan Bao ${ }^{1}$ \\ ${ }^{1}$ School of Psychological and Cognitive Sciences, Peking \\ University, China \\ ${ }^{2}$ Department of Psychology, University of California, \\ Berkeley, Berkeley, California, United States
}

Temporal information processing such as duration perception is crucial to many cognitive functions. To better estimate temporal duration, participants construct an internal reference from past experience to guide future perception. However, how the temporal reference is constructed and influenced by previous temporal experience remains unclear. In the present study, we investigated the dynamics of the internal reference from the perspective of serial dependence in temporal reproduction tasks. Participants perceived a sample duration conveyed by the presentation time of a visual stimulus. After the presentation of each sample duration, they then made reproductions by trying to hold a keypress for the same time as the sample duration. The sample durations were randomly sampled from a uniform distribution ranging from 500 milliseconds to 1.5 seconds. We found that participants' current duration reproduction is biased towards the sample duration in the previous trials. By separate experiments, we found this effect is contributed by both perceptions and motor responses, although the effect of motor response is much stronger than the perception. We proposed a novel Bayesian model with an adaptable reference to explain the serial effects observed in our duration reproduction tasks. By model simulation, we demonstrate that this new model can better predict several important properties in the serial dependence than other alternative models, namely, the adaptation-level model or the Bayesian model supposing successive stimuli being integrated by similarity.

\section{TALKS: Submission 116}

\section{Action performance in shaping conscious behavior}

Veronica Montani ${ }^{1}$, Francesco Pascucci ${ }^{2}$, Elisabetta Colombari ${ }^{1}$, Silvia Savazzi ${ }^{1}$, Paola Cesari ${ }^{1}$

${ }^{1}$ Department of Neurosciences, Biomedicine and Movement Sciences, University of Verona, Italy

${ }^{2}$ Department of Computer Science, University of Verona, Italy

According to the two-stream hypothesis, the visual input is transformed into perceptual representations in the ventral pathway while the visual control of actions is mediated by the dorsal pathway. Importantly, the visual processing mediated by the ventral stream supports visual awareness while this is not the case for the transformation of visual input into action. We investigated whether the online control of object grasping, which is mediated by the dorsal stream, could get access to information about an object' feature, i.e., orientation, of which the participant is not aware of. Participants performed a reach and grasp movement toward a briefly presented Gabor patch that could be slightly tilted either upward or downward with respect to the horizontal axis. The Gabor orientation was selected based on a previous individual threshold assessment (i.e., the orientation that participants reported they could consciously perceive about $50 \%$ of the time). Movement precision was estimated by comparing the orientations between the thumb and first digit of one hand with the orientation of the Gabor patch by means of an optical motion capture system. Results showed that participants selected the accurate hand configuration even when they reported to be not aware about the Gabor orientation, suggesting that this information could be unconsciously accessed for controlling the proper action. These findings are consistent with the two-stream hypothesis: the processing of a visual feature that is usually attributed to the ventral pathway is successfully achieved without awareness through the vision for action dorsal pathway.

\section{TALKS: Submission 117}

\section{The effect of spatial attention on temporal order perception in the visual field}

Yingrui Luo, Xin Chen, Yu Tong

School of Psychological and Cognitive Sciences, Peking University, Beijing, China 
Previous studies have shown that exogenous spatial attention can not only improve spatial resolution in visual perception tasks, but also affect the performance in temporal resolution tasks. However, these studies were limited to a few spatial locations of stimuli only and led to inconsistent results. The presented study aimed to further explore whether the spatial cueing effect on temporal order perception is asymmetric in different regions of the visual field. Using a single-cue procedure, temporal-order perception of two successive light and shade blank circles was measured. The exogenous spatial cues were either presented in the left/right visual field (Exp1) or in the upper and lower visual field (Exp2). The results showed spatial cueing effects on temporal order performance in both experiments, but only a significant visual field effect in Exp2. The temporal-order threshold was significantly lower at the cued location relative to the uncued location in both experiments, indicating a facilitation effect of attentional modulation on temporal order perception. Besides, independent of the attentional modulation, temporal-order threshold was significantly lower when the target appears in the lower visual field as compared to the upper visual field, and no such asymmetric performance was observed between the left and right visual field. These observations suggest that although the sensitivity for discriminating the temporal order of two visual events may be different in the visual field, the modulation of spatial attention on temporal-order perception is symmetric along both vertical and horizontal meridians in the visual field.

\section{TALKS: Submission 123}

\section{Subjective waiting time: better use progress bars or rings?}

\author{
Chen Zhao ${ }^{1}$, Yile Huang ${ }^{2}$, Yi Xü ${ }^{2}$, Sijia Wang ${ }^{2}$ \\ ${ }^{1}$ Institute of Medical Psychology, Ludwig-Maximilians- \\ University, Munich, Germany \\ ${ }^{2}$ School of Psychological and Cognitive Sciences, Peking \\ University, Beijing, China
}

In the digital era, people frequently experience the visualization of waiting time while downloading files, folders, or apps on mobile. The spatial visualization of waiting time is usually implemented by progress bars, rings, and so on. A few studies thus far have addressed the question of whether different progress icons with the same physical duration are experienced subjectively same or different (Kim et al. 2017; Li et al. 2021), but only with subjective questionnaires. The present study aimed to investigate this issue with an objective temporal reproduction task and further compares the potential difference between short and long waiting time while taking into account the percentage of the progress icons. Two groups of participants were asked to reproduce a short (3s) or a long (15s) duration of a progress icon respectively. Two types of icons (bar vs. ring) were combined randomly with two types of progress percentages (20\% vs. $80 \%)$. The results showed that the reproduced durations were significantly shorter with bars than with rings in the condition of long durations (15 s); But for short durations $(3 \mathrm{~s})$, there is no significant difference between the reproduced durations with bars and rings. Furthermore, independent of the shape of icons, a larger progress percentage led to longer subjective durations for both $3 \mathrm{~s}$ and $15 \mathrm{~s}$ conditions. These observations suggest that the spatial configuration matters if the waiting time is relatively long. A progress bar might reduce the subjective waiting time and thus optimize the user experience as compared to a progress ring.

\section{TALKS: Submission 126}

Do you see what I see? Exploring differing awareness of the same situation.

Graham Edgar ${ }^{1}$, D Catherwood ${ }^{1}$, S. Baker ${ }^{1}$, D. Brookes ${ }^{1}$, C. Naughtie ${ }^{1}$, G. Sallis ${ }^{1}$, G. Silcock ${ }^{1}$, S. Walker ${ }^{1}$, Y. Frampton ${ }^{1}$, R.C. Fikke ${ }^{2}$, T. Geertsema ${ }^{2}$, J. Hazebroek ${ }^{2}$, C. Tonnaer $^{2}$, R. Weewer ${ }^{2}$, A. Figueras Masip ${ }^{3}$, R. Fuste Castella ${ }^{3}$, S. Polikarpus ${ }^{4}$, M. Taukar ${ }^{4}$, T. Kütt ${ }^{4}$, F. Thoelen ${ }^{5}$, J. Vastmans ${ }^{5}$, N. Blom Andersen ${ }^{6}$, M. Bøhm ${ }^{6}$, L.O.C.N. Holm ${ }^{6}$, B. Arendtsen ${ }^{7}$

${ }^{1}$ University of Gloucestershire, United Kingdom

${ }^{2}$ IFV Institute for Safety, Netherlands

${ }^{3}$ Institute for Public Security of Catalonia (Firefighters and Civil Protection School), Spain

${ }^{4}$ Estonian Academy of Security Services, Estonia

${ }^{5}$ Provincial Centre for Education \& Training PLOT), Belgium

${ }^{6}$ University College Copenhagen (Department of Technology, Emergency And Risk Management), Denmark ${ }^{7}$ East Jutland Fire Service, Denmark

Fundamental limits on the human ability to process all available information in real-world situations necessarily require that some information is filtered out by attentional processes, and so that information is not used to build 'situation awareness' (SA). The implication is that two (or more) people in the same situation may not perceive it in the same way, nor make the same decisions as to how to operate. Such differences in perception can become very important in safety critical situations such as the one that we report here-firefighter attendance at a road-traffic collision (RTC). Using a desktop virtual reality simulation of a RTC, we examined firefighters' awareness of their situation (SA) - and also their understanding of what aspects of that situation are relevant to the successful completion of their task (situation understanding, SU). Our data, collected from 685 firefighters, suggest that the firefighters showed pronounced individual differences in the amount of information they accepted when building SA, but that they were almost universally predisposed to believe that information was relevant to their task (even though it may not have been). Non-firefighters, when compared with firefighters were generally more likely to accept a wider range of information spatially distributed across the RTC situation, but less likely to believe it to be relevant. The implication of our data is that individuals in the same situation may perceive that situation in different ways due to the way they handle the available information - and that training and experience affect that perception.

\section{TALKS: Submission 127}

\section{Speed knowledge shrink geographical representations: space-time discontinuity}

\author{
Simon Lhuillier, Valérie Gyselinck
}

Applied Psychology and Ergonomics Lab (LaPEA)Université Gustave Eiffel / Université de Paris, France

Geographical space can only be learned using symbolic representations such as maps (Montello 1993), in which it is difficult to account for both spatial and temporal information (Tversky 2000). Works in cartography attempted to accurately represent time in maps and showed that travel times shrink distances (Spiekermann \& Wegener 
1994) and speed is a main factor of space-time distortion (L'Hostis 2013). While some works explored the effect of travelling speed on spatial and temporal perception for environmental space (Riemer et al. 2018), very little is known about its impact on mental representations of geographical space. In this study, we aimed to investigate how speed information could shape metric properties of the geographical representation, and how temporal and spatial information were jointly integrated in it. To do so, we asked participants to estimate the position of cities on a map based on travel duration or distance information (reference dimension) for constant speed travels. Time and distance information could be provided along with three transportation modes: slow train, fast train, and plane (reference speed). Absolute estimated distances of the coordinates from a reference point were collected. Results show that higher speed modes (fast train and plane) lead to higher shrinking of mental geographical space when the reference dimension was time compared to space. This effect disappears for short journeys made at low speed (slow train). These results have important theoretical implication for spatial mental representations models and the question of cognitive interactions between space and time (Winter et al. 2015).

\section{TALKS: Submission 128}

\section{Playing Immersive videogames leads to estimate indoor 3D space as more accessible}

Romàn Josa, ${ }^{1,2}$, Arthur Michalland ${ }^{5,6,7}$, Anna Borghi, ${ }^{3,4}$, Arnaud Witt ${ }^{2}$, Chiara Fini ${ }^{3}$

\section{${ }^{1}$ LAPSCO-CNRS UMR 6024-Université Clermont Auvergne, Clermont-Ferrand, France \\ ${ }^{2}$ LEAD-CNRS UMR 5022-Université de Bourgogne Franche Comté, Dijon, France \\ ${ }^{3}$ Department of Dynamic and Clinical Psychology, and Health Studies, Sapienza University of Rome, Italy, Italy \\ ${ }^{4}$ Italian National Research Council, Institute of Cognitive Sciences and Technologies, Rome, Italy \\ ${ }^{5}$ Université Montpellier-LIFAM, France \\ ${ }^{6}$ Ballab-Body Action Language Laboratory, Rome, Italy \\ ${ }^{7}$ Epsylon EA4556, Montpellier, France}

Evidence showed that the body plays a crucial role in scaling our visual perception of outdoor spaces in real and 3D environments (Proffitt \& Linkenauger 2013; Fini et al. 2015, 2017). Here we try to address whether being regularly "trained" to embody an avatar can affect the 3D indoor space perception. We have selected three groups: people who do not play videogames (non players, $\mathrm{N}=44$ ), who play videogames but without embodying an avatar (not immersive players, $\mathrm{N}=21$ ), who play videogames by embodying an avatar (immersive players, $\mathrm{N}=25$ ). We designed an online study in which we asked participants to make distance and steps estimations through a visual analog scale about 3D rooms. We used linear mixed models with the distance estimation expressed in meters or by steps as dependent variable, and with the group as fixed factor. We included participants and stimuli as random intercepts. The results on steps estimations indicate a main effect of the group $(\mathrm{F}(2,87)=3.22, p<.05)$, with immersive players needing significantly less steps $(\mathrm{M}=3.3$, sd = $0.93)$ than not immersive players $(\mathrm{M}=4.2, \mathrm{sd}=1.24)$ to reach the back of the rooms $(t(87)=2.45, p<.01)$. This difference is just a tendency with non-players $(\mathrm{M}=3.9, \mathrm{sd}=1.31 ; t(87)=1.90, p=.062)$. These preliminary results suggest that, for people playing immersive videogames, indoor spaces might appear more accessible and offer an alternative virtual space of movement.

\section{TALKS: Submission 132}

\section{Emotional landmarks and spatial learning in virtual environmental space}

\author{
Téophile Rasse, Valérie Gyselinck, Jerôme Guegan
}

UMR LaPEA: Laboratoire de Psychologie et d'Ergonomie Appliquées. Université de Paris- Université Gustave Eiffel, France

The influence of emotion in spatial memory has until now received little attention in spatial cognition research. Some research has nevertheless shown that emotional properties of landmarks can enhance spatial memory (Palmiero \& Piccardi 2017) and affect the way configurational and metric properties of a route are mentally represented (Ruotolo et al. 2018). Recently, the interaction between valence and arousal of emotional landmarks on spatial memory tasks has been investigated in a Vista space (Piccardi et al. 2020). Results showed that valence, regardless of the arousal, supports learning of embodied spatial knowledge, but that the interplay between these two emotional dimensions is crucial for non-embodied spatial knowledge. The aim of the present study was also to investigate the impact of the interaction between valence and arousal of emotionally laden landmarks, but considering the metric properties of the representations of environmental spaces.Participants of the experiment in progress are instructed to watch a movie of a walk in a urban virtual environment. The routes contain landmarks that differ in terms of arousal and valence (high-arousal-positive; low-arousal-positive; high-arousalnegative; low-arousal-negative and low-arousal-neutral). Participant's spatial memory are assessed through different tasks administered with Labvanced online-experiment tool: (1)a direction recognition task evaluating route knowledge; (2)a metric and temporal length judgment; (3)a landmark-positionning task evaluating survey knowledge. In line with litterature, first results indicates that valence of emotional landmarks more than their arousal improve route knowledge and that the interplay between both dimensions influence survey knowledge acquisition and representation of distances.

\section{TALKS: Submission 139}

\section{Spatial Cognition in L2: Prepositional use among Akan-English Bilinguals}

\section{Eugene Antwi Kwarteng, Gladys Nyarko Ansah}

\section{University of Ghana, Ghana}

When learning a second language, learners are confronted with a new set of cultural and conceptual schemas that may require different cognition processes than what they have already internalized in their first languages. Studies by Boroditsky (2017) and Alonso and Jarvis (2016) have found that even in basic perceptual decisions, speakers of different languages construe space differently. In this study, bilingual spatial cognition is investigated in a Ghanaian multilingual community to ascertain whether or not Akan-English bilinguals' use of English spatial prepositions reflects Akan spatial concepts or English ones. The study adopts the cognitive grammar notion of construal to analyzes Akan-English bilinguals' description of spatial scenes that are conventionally described in English with the spatial prepositions IN, ON, and AT to represent the spatial concepts of CONTAINMENT, SUPPORT, and CO-LOCATION respectively. It was found that while the English spatial prepositions IN, ON, and AT have translation and usage equivalence with the Akan spatial prepositions MU (in), SO (on), and WO (at/ to be) in some instances but no direct equivalence in other instances, Akan-English bilinguals' use of 
English spatial prepositions were not always in agreement with English conventions for space construction. In other words, their use of English spatial prepositions reflected English spatial concepts some of the times and Akan spatial concepts in other instances. The findings of the study confirm the assertion that though the spatial concepts of CONTAINMENT, SUPPORT, and CO-LOCATION are common to most languages what counts as CONTAINMENT, SUPPORT, and CO-LOCATION varies across cultures and languages.

\section{TALKS: Submission 142}

\section{The role of culture in shaping the visual-heritage of South Indian temple cities}

\section{Gowthami Satyavarapu}

\section{M.arch sustainable urban design, India}

Through many generations, south Indian temple cities have expressed the highest aspirations in socio-cultural and economic domains, especially in the Indian context. These temple cities represent a special genre in architecture and act as independent social organisms with unique urban character. The vicinity of the temple is not confined to the central core but encloses the entire settlement, which is celebrated for continues inhabitation and adaptability to urban change. The prominent architectural structures manifest as strong cultural identities knitting visual webs.The aim of the paper was to examine how the culture of these places influenced the visual fabric of the entire settlement and how the settlement has evolved in response to it. To undertake this research, direct documentation and observations have been conducted and several samples of visual heritage have been collected for three temple cities of South India. The visual data are quantified using a 3D isovist tool, which is developed using Grasshopper plugin. To analyze the results, a multi-modal regression tool is used to examine the degree of visual influence of the temple fabric on the city's built fabric, enabling a detailed visual analysis of both the view subject and the visual corridor. The results obtained show a strong factor of dependence between culture and visual character of a settlement. Therefore, the visual fabric is one of the prominent factors that not only help in celebrating the socio-cultural identity but also enhances the sense of the belongings for a particular place.

\section{TALKS: Submission 144}

\section{Syllable discrimination based on voice onset time modulates McGurk effect}

\author{
Xuanzi Yin ${ }^{1}$, Yike $\mathrm{Li}^{2}$ \\ ${ }^{1}$ Institute of Medical Psychology, Ludwig-Maximilians- \\ Universität, Munich, Germany \\ ${ }^{2}$ School of Psychological and Cognitive Sciences, Peking \\ University, Beijing, China
}

McGurk effect refers to a speech illusion where incongruent auditory and visual syllables produce a new percept. It varies enormously among individuals, and one possible factor behind such individual difference is the extent of reliance on the auditory information. The present study aimed to further explore whether the variation of individuals' temporal processing in the auditory modality, which is critical for speech perception, might account for the individual difference in McGurk illusion. A group of university students were asked to perform three different tasks: an auditory temporal order judgment task with click sounds, an auditory syllable discrimination task where two syllables were discriminated based on the variable voice-onset-time (VOT), and a McGurk task in which a visual syllable and the same auditory syllables as in syllable discrimination task were used. The results showed that the points of subjective equality (PSE) estimated from fitted psychometric functions in auditory syllable task and McGurk task were positively correlated with each other, although the temporal order task was not directly related to the McGurk illusion. These results suggest that the temporal processing in the auditory modality may indirectly modulate McGurk effect or general audiovisual speech perception via its critical time window involved in VOT and syllable perception.

\section{TALKS: Submission 145}

\section{New Metrics and Analytical Tools for Measuring Spatial Memory}

Brent Chamberlain ${ }^{1}$, Sarah Creem-Regehr ${ }^{2}$, Jeanine Stefanucci ${ }^{2}$, Munzer Abusham ${ }^{2}$, Morgan Saxon ${ }^{2}$, Phillip Fernberg ${ }^{1}$, Brandon Garrett ${ }^{1}$

${ }^{1}$ Utah State University, United States

${ }^{2}$ University of Utah, United States

Background: Assessing navigational abilities in complex environments is challenged by the lack of systematic assessment criteria for accurately measuring spatial memory for large-scale environments, and a tedious process for measuring accuracy. Current methodologies focus on testing the recall precision of the spatial layout of elements, primarily focusing on assessing memory for routes and landmarks. Aims: The aim of this work was twofold: (1) identify a comprehensive set of spatial metrics that can assess "gist" memory or a general understanding of the spatial layout of elements and areas within a complex environment and (2) examine these metrics through new sketchmap software we developed to facilitate the automated collection of these data. Methods: We expand the use of sketchmaps to assess spatially explicit and generalizable thematic areas within a virtual environment (e.g. neighborhoods, districts, ecosystems). We create a user interface using HTML5 and develop measurement techniques using Python. The combination of the two products provides a rapid and accurate assessment of spatial memory. Results: The user interface is scalable and deliverable on a wide array of platforms. This is then combined with new metrics to evaluate spatial memory from a wayfinding task. New measures include assessing depicted layouts (shapes) and proportions of thematic districts and examining the relationship of these with landmark configuration and route memory measures. Conclusion: We have created new metrics to assess spatial memory that enables for an expanded understanding of how individuals link specific memories of objects and spaces with route and survey knowledge.

\section{TALKS: Submission 146}

\section{Vibrotactile maps for promoting spatial understanding in autonomous vehicles}

\section{Paul Fink, Nicholas Giudice}

\section{The University of Maine, United States}

Background Reimaging transportation to include fully accessible autonomous vehicles has sparked emerging research exploring the needs and preferences of people with disabilities 'at the wheel' of this new class of mobility technology. Early results from this research area suggest that haptic (touch-based) approaches seeking to increase information access to vehicle navigation information during driving are of particular interest among people who are blind and visually 
impaired (BVI). This is because haptically perceivable interfaces and maps would afford real-time monitoring and interaction with the vehicle's navigational operations, beyond current visual approaches that are inaccessible and conversational/audio approaches that are slow and periodic. Aims The solution advanced here, a new vibrotactile map interface for use in fully autonomous vehicles, poses the advantage of reducing attentional demands, thereby reducing cognitive load and enabling passengers increased time and ease of mind during transit. Currently, however, despite the advantages, research has yet to compare various approaches for conveying self-driving navigation information via non-visual cues. Methods, Results, and Conclusion This study, targeting ten BVI participants, compares participant performance (as measured by accuracy and response time) between real-time vibrotactile maps and traditional audio-based interfaces. Participants will be tasked with determining the position and route progress of an autonomous vehicle in our laboratory's simulator. Forthcoming results (expected Summer 2021) will illuminate the efficacy of new haptic interfaces for autonomous vehicles with outcomes expected to provide usability guidelines for new spatial displays that may be implemented by automotive manufacturers and stakeholders in the future.

\section{TALKS: Submission 149}

\section{Subjective time dilation in space: challenging current theories}

\author{
Dongxue Zhang ${ }^{1,2}$, Yi Wang ${ }^{2}$ \\ ${ }^{1}$ Institute of Medical Psychology, Ludwig-Maximillian \\ University of Munich, Germany \\ ${ }^{2}$ School of Psychological and Cognitive Sciences, Peking \\ University, China
}

Background: Spatial stimuli that attract more attention, such as novel or dynamic stimuli, have shown to prolong time perception. However, the subjective time dilation effect, in which a dynamic novel stimulus is only perceived to last longer when expanding in size but not when shrinking, requires further explanation. An ecological theory proposes that looming (expanding) targets are evolutionarily "threatening" as they seem to be approaching the viewer, thus inducing more arousal than receding (shrinking) targets which seem to be moving away and thus are not threatening. Another theory suggests easier attention capture playing a role, and a theory of magnitude (ATOM) proposes that sensory, spatial and temporal perception shares a common system of magnitude. Aims: To explore the effects of magnitude parameters including luminous flux (surface area $\times$ brightness) and the relative starting and finishing sizes of stimuli in subjective time dilation. Methods: We employed the psychometric method of constant stimuli and compared the subjective temporal duration of dynamic stimuli conditions with that of static stimuli. Results: We found that time dilation effect persists even when the expanding target is hollowed out or increases in brightness, but disappears when it decreases in brightness. Moreover, when the stimuli in expanding and shrinking conditions have either the same starting size or finishing size as the static stimulus, contrary to classic time dilation studies, both of them show temporal expansion. Conclusion: Current findings pose challenges to existing theories. Possibly a sustained state of attention capture can serve as a preliminary explanation.

\section{TALKS: Submission 151}

\section{HyperJump: Merging Teleporting and Continuous VR Locomotion into one Paradigm}

Bernhard E. Riecke ${ }^{1}$, Ashu Adhikari ${ }^{1}$, Daniel Zielasko ${ }^{3}$, Alexander Bretin ${ }^{1}$, Markus von der Heyde ${ }^{4,1}$, Ernst Kruijff $f^{2,1}$

${ }^{1}$ Simon Fraser University, School of Interactive Arts and Technology, Vancouver, BC, Canada

${ }^{2}$ Institute of Visual Computing, Bonn-Rhein-Sieg University of Applied Sciences, Germany

${ }^{3}$ University of Trier, Germany

${ }^{4} v d H-I T$, Germany

Locomotion is essential for all mobile beings, and effectively moving through virtual environments is becoming increasingly important. While teleporting (instantaneously jumping to a new position) can help to reduce cybersickness and decrease travel time, it can negatively impact naturalism, presence, and spatial orientation. Conversely, continuous locomotion can support path integration, selfmotion perception (vection), and thus continuous spatial updating, and provide a more real-life-like locomotion experience and higher accuracy, but can contribute to (cyber-)sickness especially when users cannot physically walk/move. Prior interfaces typically required users to switch between different locomotion control schemes, aiming for specific advantages. Here, we propose a hybrid virtual reality locomotion paradigm ("HyperJump") that aimed to combine continuous and discontinuous locomotion into one seamless interface. To investigate HyperJump, we designed an experimental paradigm combining locomotion through a series of waypoints in a naturalistic virtual city with rapid pointing to landmarks learned along novel paths ( $>300 \mathrm{~m}$ length). Locomotion was controlled either via handheld VR-controller (thumbstick-velocity-control with controller-directed steering) or a hands-free seated leaning-based interface ("HeadJoystick"). Continuous locomotion was compared with HyperJump, where intermittent jumps are automatically added whenever users reach a threshold velocity (here: $5 \mathrm{~m} / \mathrm{s}$ ), beyond which cybersickness is more likely to occur. Results showed that adding HyperJump allowed for comparable spatial orientation performance as continuous locomotion, for both controller- and leaning-based interfaces. However, leaning outperformed controller-based locomotion by reducing both orientation errors and pointing response times. We are currently improving HyperJump based on user feedback and aim to test it for larger-scale environments.

\section{TALKS: Submission 152}

\section{Contrast polarity as a factor in an attentional account of spatial compatibility}

\section{Kiril Kostov}

Department of Cognitive Science and Psychology, New Bulgarian University, Sofia, Bulgaria

In the Tucker and Ellis (1998) paradigm, keypress responses are faster when the task-irrelevant orientation of a graspable object's handle and response-hand are on the same side of space. The last two decades have pitted grasping affordance against spatial compatibility explanations in accounting for this phenomenon. More recent studies have attributed object-based correspondence effects to directional shifts in attention toward left/right hemi-space. When presented on a white background, as in previous studies, it is oftentimes the case with typical knife/fork 
stimuli that a relatively darker handle produces a stronger contrast effect against the white background, compared to the lighter-coloured, metallic functional-end. The present study aimed to investigate the extent to which object-based correspondence effects are attributed to low-level contrast polarity between the background and an object's graspable/functional end. Across three categorization experiments (using bimanual keypresses), background was manipulated (light/dark), as well as object identity (photographs vs. abstract shapes resembling knives/forks vs. non-object figures). Taken together, the results from all experiments indicated that correspondence effects were not based on grasping affordance or graspable object identity. Instead, the key driving factors which determined the direction of compatibility were background (light/dark), along with task demands. Spatial compatibility was modulated by automatic attentional capture towards areas of high local contrast, as well as top-down attentional shifts in performing the categorization tasks. These findings highlight the critical role of exogenous and endogenous attention and provide further support that the Tucker and Ellis paradigm is more suited to eliciting spatial compatibility effects, rather than grasping affordance.

\section{TALKS: Submission 153}

\section{Transition Zone: the influence of pause duration in temporal reproduction}

\author{
Nan Mü ${ }^{1}$, Jiyuan Zhang ${ }^{2}$, Yuelin $\mathrm{Liu}^{2}$, Yan Bao ${ }^{2}$ \\ ${ }^{1}$ Institute of Medical Psychology, Ludwig Maximilian \\ University, Munich, Germany \\ ${ }^{2}$ School of Psychological and Cognitive Sciences, Peking \\ University, Beijing, China
}

Subjective time is experienced differently compared to continuous physical time. In a temporal reproduction task where participants are asked to reproduce the duration of a presented stimulus, it is observed that stimulus durations shorter than approximately three seconds are usually reproduced relatively precise, while durations longer than three seconds tend to be underestimated. However, all these previous studies used only a short time delay between the offset of the standard stimulus and the onset of reproduction. The present study aimed to further investigate whether the length of pause duration might influence participants' performance in a temporal reproduction task. Three different standard durations $(2 \mathrm{~s}, 3 \mathrm{~s}, 4.5 \mathrm{~s})$ and various pause durations (from $1 \mathrm{~s}$ up to $36 \mathrm{~s}$ ) were represented in a series of experiments. Different groups of participants were asked to reproduce previously perceived standard durations by pressing a key to terminate the duration of a reproduced stimulus. The results showed a significant linear increase in reproduced duration as the pause duration increased. However, when the pause duration exceeded approximately $3 \mathrm{~s}$, a plateau of the same reproduced duration was observed. These observations suggest that pause duration plays a critical role in the temporal reproduction process. A time window of approximately three seconds serves as a transition zone for reaching a stable internal temporal reference in temporal perception.

\section{TALKS: Submission 154}

\section{Does linear perspective accurately depict how we perceive distance?}

Joanna Ganczarek ${ }^{1}$, Nicole Ruta ${ }^{2}$, Karolina Pietras ${ }^{1}$, Alistair Burleigh ${ }^{3}$, Robert Pepperell ${ }^{4}$

${ }^{1}$ Pedagogical University of Cracow, Institute of Psychology, Poland

${ }^{2}$ University of St. Andrews, St. Andrews, United Kingdom

${ }^{3}$ Fovotec, Cardiff, United Kingdom

${ }^{4}$ Cardiff Metropolitan University, Cardiff, United Kingdom Background: The known limitations of linear perspective have led artists to develop alternative methods of depicting their subjective experience of space, such as various forms of so-called 'natural perspective' based on the phenomenological structure of human visual space. Previous studies have shown that natural perspective projections outperform linear perspective ones in terms of aesthetic preferences, enhanced sense of depth, and immersion. Aims: We tested whether natural perspective projections outperform linear perspective in terms of distance estimates in a virtual 3D environment. Methods: We conducted two studies in which the effects of linear versus natural perspective on non-metric distance judgements were tested. The participants in both experiments judged distances in 72 linear perspective and natural perspective images in total, rendered with three different fields-of-view: 100, 120 and 140 degrees horizontally. In the second experiment we additionally tested the influence of formal education in linear perspective, experimental training, and the impact of spatial skills on distance estimates. $R e$ sults: The results of both experiments confirmed our hypothesis that natural perspective improves distance estimation, particularly at wideangle fields of view. Moreover, experimental training with natural perspective images led to less overestimations in natural perspective images compared to linear perspective ones. Finally, spatial skills predicted positively accuracy in distance estimation, albeit the effect was very small. Conclusion: Together, our findings suggest that natural perspective improves distance estimation, and this remains so for those with formal training in linear perspective.

\section{TALKS: Submission 155}

\section{Evolution of spatial decentration and language comprehension among young children}

\author{
Natacha Duroisin ${ }^{1}$, Hursula Mengue-Topio ${ }^{2}$ \\ ${ }^{1}$ University of Mons, Belgium \\ ${ }^{2}$ University of Lille, France
}

This study focuses on the link between Spatial Perspective Taking (SPT) and receptive language in children between 4 and 7 years. SPT corresponds to the ability to differentiate one's own point of view from that of another observer and then to represent the point of view of this other observer in a "visual scene" (Beaudichon \& Bideaud 1979). From 3 to 4 years, children are able to judge whether an object would be seen or not by adopting another point of view (Sodian et al. 2007). We question the link between the understanding of basic concepts in children and theirs results in a task assessing SPT. Despite the development of SPT does not require mastery of verbal skills (Greenberg et al. 2013), the child must nevertheless understand the instructions presented. However, it is between 3 and 5 years that the understanding of basic language is acquired (Miljkovitch et al. 2017). We examine performance of decentration while considering the 
understanding of the concepts. Our predictions: spatial judgments errors decrease as participants get older and we expect a decrease in errors in children with a high score in the test of language. Participants: 60 children (4-7 years). Material: Boehm to assess the development of receptive language and Animo-Déclic ${ }^{\circledR}$ game to assess the SPT. For SPT, we note a significant decrease in the number of errors according to the participants' age (Kruskal-Wallis Test $(\mathrm{N}=$ $60)=34.62, p<0.001)$. Results show strong and negative links between understanding language basic concepts and errors in SPT task $(p<.001)$. We show an improvement in SPT performance between 5 and 6 years with a significant reduction in the number of errors at 6 years 11 months.

\section{TALKS: Submission 160}

\section{Sonic Environments: Localization of Sounds and Re- localization of Their Hearer}

\section{Martin Nitsche}

\author{
Institute of Philosophy, Czech Academy of Sciences, \\ Prague
}

Background: Our life-environment is increasingly affected by sounds, noises, voices, and music. With the notion of "sonic environment," I designate, in this proposal, precisely this acoustic shape of our lifeenvironment. Sonic environments are not composed of sounds as separate objects, but created by lived sonic phenomena. Essentially, these phenomena are understood as relations between sounds and their hearer. In this talk, my goal is to explain spatial aspects of these relations. I will mainly investigate how these relations are localized: whether a hearer relates two previous localizations (of a sound and herself) or whether a sonic phenomenon can be localized as a relation. Aims: How do sounds-hearer relations create sonic environments? Are sounds localized primarily with sounding objects or as parts of sounds-hearer relations? How hearing affects the localization of a hearer? Methods: Phenomenological analysis in the Husserlian sense, i.e. including phenomenological reduction to lived experience as a stream of consciousness. Results. Based on a phenomenological analysis of hearing, I will claim that: 1 . sonic phenomena are localized primarily as sounds-hearer relations (similar to topological relations); 2 . in this relational framework, a hearer's localization must be understood as a re-localization (with respect to a natural centric ego-localization). Conclusion: In order to properly describe sonic environments, we must pay attention to relational co-localizations of sounds and their hearer. Lived aspects of sonic environments are tied with a hearer's re-localization generated by the relational co-localization of sounds.

\section{TALKS: Submission 161}

\section{Spatial perspective taking in children: the effect of oblique orientation}

\author{
Hursula MENGUE-TOPIO ${ }^{1}$, Natacha Duroisin ${ }^{2}$ \\ ${ }^{1}$ Univ. Lille, ULR 4072-PSITEC-Psychologie: Interac- \\ tions, Temps, Emotions, Cognition, Lille, France \\ ${ }^{2}$ Univ. Mons-École de Formation des Enseignants, 7000, \\ Belgium
}

This developmental study explores the effect of oblique orientation on spatial perspectives taking (SPT) in children between 5 and 9 years old. SPT is the ability to represent how an object or array of objects looks from other viewpoint and has been studied in developmental psychology since Piaget's Three Mountains task (Piaget and Inhelder 1956).Most studies have assessed SPT by asking children to to look at a model layout of objects and to judge how an observer would see this layout from different angles $\left(0^{\circ}, 90^{\circ}, 180^{\circ}, 270\right)$. The focus of the current study is to assess the effect of non-canonical orientations such as oblique $\left(45^{\circ}, 135^{\circ}, 225^{\circ}\right)$ in spatial reasoning in children. Using a playful (Animals and guardians of zoo have taken pictures) and increasingly complex learning situation (3 Spatial orientations, 16 animals, an internal and external point of view of the zoo)we examine the integration of different spatial orientations (front-to-back, leftright, oblique) during spatial reasoning in children aged 5-9 years. The results show a low number of errors and stable performance of children aged 7-9, regardless of the spatial orientation studied. In contrast, children aged 5 and 6 make more errors in judgment, especially for oblique orientation compared to other orientations (Anova chi2 $(2, \mathrm{~N}=95)=13,5, p<0.001)$. These results suggest a different sensitivity, depending on the age of the participants, to the characteristics of the tasks assessing spatial perspectives taking.

\section{TALKS: Submission 162}

\section{Cognitive Modelling of Visuospatial Complexity in the Streetscape}

Vasiliki Kondyli, Mehul Bhatt

Orebro University, Sweden CoDesign Lab > Cognitive Vision, Sweden

Background: Incorporating knowledge about human behaviour and the effect of the environment is a major goal for the design and engineering of human-centred autonomous vehicles. Systems that aim to establish a common interaction ground with humans require systematic modelling of empirically established behavioural norms customised to specific contexts. Aims: Focusing on aspects pertaining to visual attention in driving, we develop a cognitive model of visuospatial complexity for naturalistic driving scenes and explore its effect on visual attention tasks (e.g., involving visual search) during everyday driving. Methods: By analyzing dynamic naturalistic scenes, we define a scale of visuospatial complexity based on a taxonomy of quantitative, structural, and dynamic attributes. We re-create realworld instances in virtual reality (VR) in four levels of visuospatial complexity. The human-centred basis of the model lies in its behavioural evaluation with human subjects with respect to psychophysical measures (e.g. eye-tracking) pertaining to embodied visuospatial attention. Results: Empirical results show the levels of visuospatial complexity of the scene correlate with visual search performance parameters, however different categories of attributes contribute differently to the overall effect. We report work-in-progress on the development of a (sample) dataset with the central emphasis on the evaluation of the visuospatial complexity levels on driving stimuli within VR. Conclusion: The presented cognitive model of visuospatial complexity in everyday driving situations can be used as a basis to design, and evaluate visuospatial sensemaking capabilities of autonomous vehicles. We posit that our methodology encapsulates key cognitive principles founded on empirically established behavioural patterns under naturalistic conditions. 


\section{TALKS: Submission 163}

\section{Impact of symmetry type and axial direction on interface layout preference}

\author{
Lìga Zariņa ${ }^{l}$, Jurğis Šǩilters ${ }^{l}$, \\ Aisha F. Tüchler ${ }^{l, 2}$ \\ ${ }^{1}$ Laboratory for Perceptual and Cognitive Systems, \\ University of Latvia, Riga, Latvia \\ ${ }^{2} e-L A D D A$, Norway
}

Symmetry properties are crucial in object perception (Wagemans 1997), preference, and recognition (e.g., Beramini et al. 2019). According to research on UX, certain principles of interface design linked to geometric properties, such as principles of readability, visual hierarchy, and Gestalt principles, amongst others (Hartson \& Pyla 2012), are commonly applied to improve the user's interaction experience and are therefore familiar to users.In our study, the impact of different symmetry types and axial directions on user's preferences has been systematically tested. Participants evaluated canonical interface layouts such as columns, boxes, and different types of grids, which were presented to them in varying symmetry conditions, such as reflection, rotation, and translation, amongst others. We conducted an online experiment, employing an in-between group design $(\mathrm{N}=$ $65)$, where symmetry properties were applied to the experimental stimuli to test how these properties relate to the most and less preferred layouts. Additionally, we analysed the users' perceptions of the layout types according to aesthetic and usability criteria (Lavie \& Tractinsky 2004), and explored their perceived appropriateness for different interface use (e.g., education, medical, entertainment). Our study reveals that even highly symmetric layouts are not always preferred, hinting towards other features being influential/decisive when evaluating interface usability. These influential features will be comprehensively outlined in this contribution, and aid to understand symmetry as an important visual design feature, which might inform future design of user interfaces.

\section{TALKS: Submission 170}

\section{Linking the design of university learning spaces to students' experience}

\section{Beatrix Emo ${ }^{1,2}$, Gregoire Farquet ${ }^{l}$, Momoyo Kaijima ${ }^{l}$, Christoph Hoelscher ${ }^{1}$ \\ ${ }^{1}$ ETH Zurich, Switzerland \\ ${ }^{2} Z H A W$, Switzerland}

Background: The paper draws on research at the intersection of spatial cognition and architectural design to examine how the design of a space can influence how people behave in it. We examine the potential of well-designed informal learning spaces for university students. The distinction of formal versus informal learning spaces comes from the learning sciences; whereas formal learning spaces are primarily designed for learning (e.g. classrooms), in informal learning spaces students typically combine learning with other activities, such as eating, drinking and socialising. Aims: To examine how students' experiences of informal learning spaces are affected by the design of the space. Methods: We present findings from an online survey on how students experienced a set of temporary informal learning spaces. Spaces were rated for the following attributes: atmosphere, temperature, acoustics, light, electricity, wifi and accessibility. Additional survey questions provide more general feedback about how students experience the spaces. Results: Findings show that students rely on informal learning spaces for their studies and that they need more such spaces. While their basic learning needs are met in the current design, they expect a more comfortable atmosphere. Together with findings from social network studies, we argue that students' learning experiences are improved when the spaces are well-designed. Conclusion: We relate the findings from this study to broader spatial cognition literature on how architectural design can align the intended and actual behaviour of individuals in space. We discuss the potential gain of designing more comfortable spaces that better fit with student users' expectations.

\section{TALKS: Submission 172}

Influence of hand action on the processing of symbolic numbers.

Mariagrazia Ranzini ${ }^{1}$, Carlo Semenza ${ }^{2}$, Marco Zorzi ${ }^{3,4}$, Simone Cutini ${ }^{5}$

${ }^{1}$ Department of Neuroscience, University of Padova, Italy ${ }^{2}$ Department of Neuroscience (Padova Neuroscience Center), University of Padova, Italy

${ }^{3}$ Department of General Psychology (DPG), University of Padova, Italy

${ }^{4}$ IRCCS San Camillo Hospital, Venice-Lido, Italy

${ }^{5}$ Department of Developmental Psychology and Socialisation (DPSS), University of Padova, Italy

Background: Number-space and number-size interactions are welldocumented, and many studies suggest that they are grounded into sensorimotor mechanisms. In line with embodied and grounded cognition accounts, behavioural studies have described interactions between number processing and the hand action of reaching and grasping objects in space. Aims: In this study, we explored the effects of repetitive hand actions on number comparison. Based on previous studies, we hypothesised that hand grasping and pointing, but not hand tapping or viewing (object looking), would impact performance in number comparison. Methods: Participants $(\mathrm{N}=24$; mean age $=$ 23ys) performed repetitive object grasping (16 times), repetitive pointing, repetitive tapping, or object looking. Subsequently, participants performed a number magnitude comparison task (comparing digits with 5). The design consisted of three within-subject factors: Action Type (Grasp; Point; Tap; Look), Number Magnitude (small or large: $1-4 ; 6-9$ ), Number Distance (far or close to the reference: 1-2, $8-9 ; 3-4,6-7)$. This study research plan was preregistered on the Open Science Framework (OSF) prior to data collection. Results: We found that executing the action of pointing slowed down the speed of response in number comparison. Moreover, with small numbers only, the typical distance effect (faster responses for numbers far from the reference as compared to close ones) was not observed after pointing, while it was enhanced by grasping. Conclusion: These findings confirm the functional link between hand action and number processes, and permit to advance new interesting hypotheses on the role of pointing - besides reaching and grasping - in the embodiment of numerical skills. 


\section{TALKS: Submission 173}

\section{Is non-nutritive sucking a tool for blind infants to map space? an EEG study}

Helene Vitali ${ }^{1}$, Claudio Campus ${ }^{1}$, Valentina De Giorgis ${ }^{2}$, Sabrina Signorini ${ }^{2}$, Federica Morelli ${ }^{2}$, Marco Fasce $^{2}$, Monica Gori ${ }^{1}$

${ }^{1}$ U-VIP: Unit for Visually Impaired People, Istituto Italiano di Tecnologia, 16152, Genova, Italy

${ }^{2}$ IRCCS Mondino Foundation, 27100, Pavia, Italy

Brain plasticity mechanisms and the development of specific networks occur mostly in the first years of life. These mechanisms partly fail in blind children and may impair different spatial tasks, which is also reflected in delayed development of motor coordination and reduced spatial exploration. However, no data exist on motor activity modulation in the first years of life in blind infants. This work investigates the modulation of motor activity and its relationship with different cortical rhythms in sighted and blind infants/toddlers. In particular, we focused on the non-nutritive sucking (NNS) which is one of the first coordinated muscular activities and is an excellent marker of neural system functioning also in the first stages of life. We investigated whether the NNS is only a motor activity that leads to idling status or if it can also be involved in the spatial representation process. We retrospectively compared spectral EEG activity of blind subjects with [6-48] months and matched sighted controls. We found that associations of NNS with ERSP in alpha and beta EEG bands specifically distinguished groups. Sighted subjects showed an idlingsynchronization mechanism with the formation of beta 1 band bridge along the visual-motor pathway. Conversely, blind subjects showed an attentive-desynchronization mechanism in alpha 1 band. These findings suggest a plastic reorganization of circuits involved during NNS assuming a different function, like space representation behaviour since the specificity of dorsal area. Nevertheless, this reorganization is to the detriment of the visual-motor pathways indicating possible future motor impairment.

\section{TALKS: Submission 174}

\section{Embodied VR Flying Improves Spatial Orientation while Reducing Cybersickness}

\author{
Ashu Adhikari ${ }^{1}$, Bernhard E. Riecke ${ }^{1}$, \\ Abraham M. Hashemian ${ }^{1}$, Thinh Nguyen-Vo ${ }^{1,2}$, \\ Ernst Kruijff ${ }^{3,1}$, Markus von der Heyde ${ }^{4,1}$ \\ ${ }^{1}$ Simon Fraser University, School of Interactive Arts and \\ Technology, Vancouver, BC, Canada \\ ${ }^{2}$ Microsoft, Redmond, WA, United States \\ ${ }^{3}$ Institute of Visual Computing, Bonn-Rhein-Sieg Univer- \\ sity of Applied Sciences, Germany \\ ${ }^{4} v d H-I T$, Germany
}

Supporting low-cognitive-load spatial orientation while reducing motion sickness are key challenges for locomotion in virtual reality (VR) applications. For ground-based locomotion, we have physical walking as the "gold standard", but for 3D (flying) there is no such equivalent. Here, we investigated if replacing the standard 2-thumbstick flight controllers with an embodied (leaning-based) flying interface ("HeadJoystick") could help improve user experience and performance. To investigate this, we designed a gamified 3D (flying) navigational search paradigm where 22 participants ( 10 female) had 5 minutes to find 8 objects hidden in 16 boxes randomly positioned in a landmark-free 3D virtual environment presented immersively via HMD (HTC Vive). Virtual translation was controlled by either leaning (forward/backward, left/right, up/down) or using the Gamepad's thumbsticks. Rotations were always performed by participants physically turning on their swivel chair. Compared to using thumbsticks, leaning-based locomotion improved performance (more collected balls while reducing distances traveled) while also reducing motion sickness and mental task demand. Although HeadJoystick was rated as more physically fatiguing for long-term use, participants felt more engaged, enjoyed it more, and overall preferred it. Together, this suggests that providing translational proprioceptive/vestibular cues using a seated leaning-based interface (HeadJoystick) can provide an effective, engaging, and no-additional-cost alternative to the conventional thumbstick-based flying interfaces, and help to improve spatial orientation and spatial updating in 3D space. Furthermore, HeadJoystick frees up user's hands and to some degree their minds, so their hands can more easily be used for other tasks such as interaction or communication.

\section{TALKS: Submission 177}

An online tool for the detection of pathological spatial asymmetries

\author{
Giulio Contemori ${ }^{1}$, Maria Silvia Saccani ${ }^{1}$, Elvio Blini ${ }^{1}$, \\ Zaira Romeo $^{l}$, Marco Zorzi ${ }^{1,2}$, Mario Bonato ${ }^{l}$ \\ ${ }^{1}$ Department of General Psychology, University of Padova, \\ Italy \\ ${ }^{2}$ IRCCS San Camillo Lido-Venice, Italy
}

Background: In the last decade, we have been successfully using a multitasking approach designed to unveil spatial asymmetries in the processing of lateralized visual stimuli. It couples a single task, spatial only condition, with two multitasking conditions requiring the concurrent processing of visual or auditory stimuli. Its effectiveness in detecting unilateral spatial omission (neglect/extinction) in apparently unimpaired patients - be them chronic or acute, left or right brain damaged-has now been established by multiple labs. Aims: We implemented an online adaptation of task version by Blini et al. (2016) and recruited a group of healthy elderly participants $(\mathrm{N}=13)$ to test its feasibility and obtain reference data. Methods: This free online tool does not need a dedicated software-it is based on JS Psych and runs entirely in a browser. The task can be easily administered by a caregiver to participants speaking any language. It is complemented by an intuitive graphical interface that quantifies, visualizes and statistically tests the presence of spatial asymmetries. Results: As expected, performance of healthy participants in the primary task was close to the ceiling (accuracy 97\%). A slight advantage for left-sided stimuli emerged across conditions. In the secondary task, performance was lower for the visual than for the auditory modality. Conclusion: In conclusion, the online adaptation provided reference data useful for a direct comparison with brain damaged patients. The very good performance confirms its suitability for the testing of the elderly. Task variants for research with healthy participants will be discussed. 


\section{TALKS: Submission 183}

\section{Time as space metaphors in Chinese Sign Language}

\section{Juan Sun}

\author{
School of Foreign Languages, Sun Yat-sen University, \\ China
}

Spatial metaphors reveal how we understand abstract concepts such as time (Lakoff and Johnson 1980). Due to the use of both front/back and up/down spatial terms to talk about time, the way that Chinese speakers conceptualize time has been widely investigated (Boroditsky 2001; Yu 2012). However, previous studies have focused only on Chinese people's spoken language, without paying much attention to their sign language. Sign languages have both linguistic and gestural components, providing thus a unique angle for investigating spatial metaphors (Börstell \& Lepic 2020). The aim of this study was to investigate spatiotemporal metaphors in Chinese Sign Language (CSL) and to provide further information regarding the mental spacetime mappings in Chinese people. To do so, we conducted a manual analysis of 50 signs expressing temporal notions extracted from the CSL Dictionary (published in 2019). According to the results, there are directional movements in the front/back (25/50), left/right (8/50) and up/down (17/50) axes. In the vertical up/down axis, we noticed "upward-earlier, downward-later" metaphorical mapping in CSL as in the spoken language. In the horizontal front/back axis, we observed only future-in-front metaphors in CSL, differently from the spoken Chinese in which both the future-in-front and past-in-front metaphors exist. Concerning the left/right axis, we found several spatial metaphors but failed to confirm the "leftward-earlier, rightward-later" temporal schema addressed by previous studies on Chinese speakers' temporal gestures (Li 2017). In summary, the results obtained show that spatiotemporal metaphors are abundant and multidimensional in CSL and suggest the existence of upward/forward-earlier space-time mappings.

\section{TALKS: Submission 184}

\section{Situated cognition, mental imagery and memory strategies}

\section{Quentin Marre, Nathalie Huet, Elodie Labeye}

CLLE Laboratory (Cognition, Languages, Language, Ergonomics), UMRS 5263-CNRS, University of Toulouse Jean Jaurès, Toulouse, France

Background: How much can our sensorimotor processes help our memory? In a previous study, we demonstrated the effectiveness of an imagery strategy involving both visual and motor characteristics in a first person view (compared to mental rehearsal, visual imagery and third person visuo-motor imagery). These results support the embodied cognition paradigm according to which memory retrieval requires a reenactment of the sensory and motor dimensions of the encoding experience. According to this paradigm, cognition is also situated, i.e. when knowledge (e.g. of a chair) is activated, it is simulated in the context of probable background situations (e.g. a kitchen). Aim: In the present study, we therefore wanted to test whether motor imagery could be made more memory efficient by adding a contextual dimension. Methods: With an incidental learning task, we compared the free recall and recognition performance of 229 participants using four strategies: mental rehearsal (control condition without imagery), visual imagery, motor imagery (imagining oneself throwing the object) or situated motor imagery (imagining throwing the object while being in a situation consistent with the object). $R e$ sults: The results of this study showed a significant effect of the strategy type on free recall, with situated motor imagery being the most memory effective. Conclusion: The results corroborated the main hypothesis, in line with an embodied and situated nature of memory. Memory appears to be designed to support situated action as memory traces explicitly supporting a situated action are easier to retrieve than traces that are less useful to support a situated action.

\section{TALKS: Submission 191}

\section{The role of orientation within complex historical urban contexts in Italy}

\section{Giulia Mastrodonato, Domenico Camarda}

Polytechnic University of Bari, Bari, Italy

This work investigates the role of orientation in the decisions of agents navigating urban spaces with complex topological layouts. The final aim was to support spatial planning for historic centers - an important part of the European urban policy agendas. The paper uses an experimental approach to investigate the behavior of agents' wayfinding within the historic town of Martina Franca, southern Italy. The knowledge deriving from direct learning of the environment is often reported as the result of sensory information coming from human body during locomotion in relation to the environment's characteristics and shape. Among the variables influencing this perception, here we look particularly at the length of the path and the number of its segments, the environmental distance beyond our visual field and the incidence of the angularity of turns on the ability to correctly perform a wayfinding task. The agents involved in the experimentation are Spatial Planning students from the Polytechnic of Bari, Italy. The exploration takes place during the lockdown period for the Covid-19 emergency. In this period the empty spaces allow possible reductions of navigation variables and more concise modelling of the spatial cognitive relationship process. Yet, the experimentation context may not be well representative of a typical urban situation, if (hopefully) it will return to normality in the near future. Even in a rather contingent context, the results should represent useful baselines for more articulated research follow-ups about the issues of orientation of navigating agents, as well as of spatial-cognitive planning.

\section{TALKS: Submission 196}

\section{Spatial ability, scientific reasoning, and graphing skills} in kinematics

\section{Rachel Harding}

\section{Technological University Dublin, Ireland}

Background: Approximately 300 Junior Cycle science students (12-15 years old) in Ireland participated in this study. Aims: The aim of the study was to investigate the relationship between junior cycle science students' spatial ability, scientific reasoning, and understanding of graphing in kinematics, and how this changes over the first three years of second level education. Gender differences in abilities were investigated. Methods: The Mental Rotation test (MRT), the Lawson Classroom Test of Scientific Reasoning (CTSR), and the Test of Understanding Graphing in Kinematics (TUG-K2) were administered to class groups of Junior Cycle science students as part of their normal science class sessions. The resulting data was analysed solely by the researcher. Results: The preliminary findings from this study showed a significant gender difference favouring males in spatial ability and scientific reasoning across all three years of Junior Cycle. There was no significant gender differences in the 
test of understanding graphing in kinematics. The findings showed that the results were close to chance (20\%) across all 3 years of study, indicating that the students were not improving on key graphing skills in a kinematics context. This is a concerning finding in the Irish second level context. Conclusion: The preliminary findings warrant further investigation into why the gender gap favouring males in spatial ability and scientific reasoning appears to be increasing from 12 to 15 years old, and why the students' skills in kinematics graphing show no improvement across Junior Cycle science. This study is part of the researchers' Ph.D. Thesis.

\section{TALKS: Submission 197}

\section{Review on Techniques to Measure Spatial Affordances in VR Environment}

\section{Tania Miranti Chumaira, Lily Díaz-Kommonen, Luis Emilio Bruni}

\section{Aalto University, Finland}

Aalborg University, Denmark

In the past years, research progress on virtual reality (VR) has shifted its focus to measuring our spatial experience within the VR environment concerning its spatial affordances instead of creating new designs and modes of interaction. However, most scholars heavily lie on a quantitative approach to measure and understand spatial affordances in the VR environment, which treats subjective human experiences as fragmented sequences. Therefore, we conduct a literature review of techniques used by most scholars in VR research to understand spatial affordances, techniques borrowed from the field of architecture to read spatial affordances, and critically discuss the potential of implementing such techniques in studying spatial affordances in VR environment. The study reveals the potential in creative mapping that can provide insights related to spatial affordances as a whole event and how it is linked to meaningful events experienced by users while capturing the granularity of subjective human experience. This review suggests that creative mapping can reveal the narrative of the virtual experience, highlights of the spatial experience, sensorial sensation, and when these factors emerge. This way, the review suggests that creative mapping allows us to understand the link between body trajectory and events. Finally, this study helps us fill a gap in the current focus, which is more oriented towards the quantitative approach.

\section{TALKS: Submission 200}

\section{Humans Select Informative Views Efficiently To Recognise Actions}

Carmelo Calafiore $^{1,2}$, Tom Foulsham ${ }^{1}$, Dimitri Ognibene ${ }^{2}$

${ }^{1}$ Department of Psychology, University of Essex, Colchester, United Kingdom

${ }^{2}$ School of Computer Science and Electronic Engineering, University of Essex, Colchester, United Kingdom

Background: Humans and robots must understand each other to interact. Previous studies have shown that both humans and robots can easily recognise social stimuli such as facial expressions or actions. However, very few studies have explored their social proactive vision (SPV). We define SPV as the skill of selecting the most informative views in 3D space to recognise social stimuli. Aims: We investigated whether and how humans can choose informative viewpoints efficiently to recognise actions. Methods: Human and robotic participants were asked to classify human actions from different viewpoints. The robotic participants were Artificial Neural Networks (ANN). In the non-movement condition, human and robotic participants could not move/select the view. In the random movement condition, only human participants looked at the actions from a view that changed randomly. In the selfcontrolled movement condition, only human participants were able to move/select their viewpoint by themselves. Results: In the nonmovement condition, human and robotic participants performed better from the top, front and side views with respect to the actor executing the action. In the self-controlled condition, the human participants selected the top, front and side views more often. The human performance of action recognition was better in the self-controlled movement condition than in the other two conditions. Conclusion: Humans select viewpoints efficiently to recognise actions. Because of two reasons. One, they selected more often the informative views which were the top, front and side views. Two, their action recognition performance improved when they could select their viewpoint.

\section{TALKS: Submission 204}

Travel in the physical and mental spaces of navigation and memory

\section{Agustina Fragueiro, Annalisa Tosoni, Matteo Frisoni, Rosalia Di Matteo, Carlo Sestieri, Giorgia Committeri}

Department of Neuroscience, Imaging and Clinical Sciences, University G. d'Annunzio, Chieti-Pescara, Italy

Background: Based on the neuro-functional association between navigation in the physical and the mental space at the level of the hippocampal-entorhinal system, it has been hypothesized that mechanisms of episodic memory and semantic memory evolved from mechanisms of self-based and map-based navigation in the physical space, respectively. We recently showed a correlational association and a predictive relationship between human abilities in egocentric navigation and episodic memory. Aims: To confirm and extend this evidence to the dynamic component of both self-based egocentric navigation and episodic memory, and at better describing their functional relationship with semantic memory functions. Methods: Three experimental tasks were designed in which the dynamic component of time travel and sequential updating of information was emphasized in both the spatial (Travel in Space), the temporal (Travel in Time), and the semantic (Travel in Categories) domain. The working memory contribution to the three tasks was controlled by including a working memory task with an analogous structure. Results: The results confirmed the existence of a direct and predictive relationship between self-based spatial navigation and episodic memory. The results additionally showed the presence of a significant association between egocentric navigation and semantic memory but this relationship was explained by working memory abilities and, more importantly was mediated by episodic memory functions. Conclusion: Once more, our results support the hypothesis of an evolutionary link and a phylogenetic continuity between mechanisms of spatial navigation and time travel in the physical and in the mental space. 


\section{TALKS: Submission 210}

Reaching to sounds to improve spatial hearing: a visual virtual reality approach

Chiara Valzolgher ${ }^{1,2}$, Michela Todeschini ${ }^{3}$, Gregoire Verdelet ${ }^{1,6}$, Romeo Salemme ${ }^{1,6}$, Valerie Gaveau $^{1,4}$, Eric Truy ${ }^{5}$, Alessandro Farne ${ }^{1,2,6}$, Francesco Pavani ${ }^{1,2}$

${ }^{1}$ Integrative, Multisensory, Perception, Action and Cognition Team (IMPACT), Lyon Neuroscience Research Center, France

${ }^{2}$ Center for Mind/Brain Sciences (CIMeC), University of Trento, Italy

${ }^{3}$ Department of Psychology and Cognitive Sciences (DiPSCo), University of Trento, Italy

${ }^{4}$ University of Lyon 1, France

${ }^{5}$ Hospices Civils de Lyon, Lyon, France

${ }^{6}$ Neuroimmersion, Lyon, France

Background: Spatial hearing changes when the peripheral auditory cues are altered (e.g. hearing impairments). Learning new correspondences between auditory cues and spatial coordinates is still possible during adulthood. When relearning, multisensory stimulation and feedback play key roles, as well as active interaction with the sounds (head-movements, hand-reaching). Aims: Using sound localization tasks (1) test the immediate and generalization effects of reaching to sounds in adapting to altered auditory cues (monaural listening); (2) measure changes in head-movement behaviour during listening. Methods: We proposed a new approach based on virtual reality and head-motion tracking. We examined spatial hearing adaptation in 20 normal-hearing participants with one ear plugged by testing them in a head-pointing sound localization and in an audiovisual cueing task before and after participating in a short training paradigm. We compared a spatial training, in which participants localized sound sources by reaching them, to a non-spatial training, in which participants received identical auditory stimulation, but identified the type of the sound irrespective of its spatial position. Results: We found that reaching to sounds rapidly improves spatial hearing when auditory cues were altered, and its benefit generalizes to the head-pointing sound localization task, but not to the audio-visual cueing task. Interestingly, learning to localize sounds is associated with the implementation of novel head-orienting strategies. Conclusion: Our findings suggest a contribution of active behavioural strategies (reaching to sounds, head-orienting behaviour) when adapting to altered auditory cues, with implications for training people that perceive auditory space through assisted hearing (hearing aids or cochlear implants).

\section{TALKS: Submission 211}

Virtual Kitchen 2.0: comparing different tutorials approaches in VR training

Giada Corrias ${ }^{1}$, Rachele Fanari ${ }^{1}$, Franco Delogu ${ }^{2}$, Stefano Curatti ${ }^{3}$

${ }^{1}$ Università degli Studi di Cagliari, Cagliari, Italy

${ }^{2}$ Lawrence Technological University, Michigan, United States

${ }^{3}$ Infora Soc. Coop., Cagliari, Italy
The availability to the broad public of progressively more advanced Virtual Reality (VR) devices underline the need for further research about their effectiveness in educational and training contexts, aiming at the creation of guidelines for the design of effective environments for training and teaching. Current literature lacks specific studies of the pedagogical effectiveness in terms of quality and speed of learning determined by different structural feedback elements. In VR training, an ergonomic design of all the environment's elements, and of the feedback pedagogical elements, would help users in the use of interfaces and designers in the calibration of user frustration and in the identification, comment, and highlight of key relationships at the appropriate time. We developed Virtual Kitchen (VK), a VR software to test different combinations of feedback and their effectiveness in a fully immersive virtual reality environment. In this study, participants were asked to prepare a dessert in VK following either written instructions (texts condition) or iconic instructions (icons condition). Using a commercially available head-mounted display and two controllers, 23 students (11 female) from Lawrence Technological University performed 7 tasks, simulating the steps to prepare the dessert. The tasks were performed either in the "icons" or in the "text" condition. Two weeks later they performed the tasks again in the same conditions. Preliminary results show that participants in the "icons" condition were faster and more accurate than the subjects exposed to the "texts" condition. Moreover, the "icons" condition led to a better memory of the procedure after 2 weeks.

\section{TALKS: Submission 214}

\section{Mind in Motion: How Action Shapes Thought}

\section{Barbara Tversky}

\section{Stanford University}

All living things must move and act in space to survive, even plants, In primates, the same brain structures that support spatial thinking also support conceptual thinking, This is evident in the ways we think and the ways they externalize thought, primarily through words, gestures, and graphics, Our minds go from thought to thought the ways our feet go from place to place, Our words and gestures act on thought the way we act on objects: we raise ideas, pull them together, tear them apart, Gestures and graphics bear more direct relations to meanings than symbolic words. They alter our own thinking and that of others, Our actions in the world design the world, putting our minds in the world.

\section{Abstracts of Posters}

\section{POSTERS: Submission 4}

To touch or not to touch? EEG correlates of an in-store sensory experience Laura Angioletti ${ }^{1,2}$, Roberta Sebastiani ${ }^{3}$, Michela
Balconi $^{1,2}$

${ }^{1}$ International Research Center for Cognitive Applied Neuroscience (IrcCAN), Faculty of Psychology, Catholic University of the Sacred Heart, Milan, Italy

${ }^{2}$ Research Unit in Affective and Social Neuroscience, Department of Psychology, Catholic University of the Sacred Heart, Milan, Italy 
${ }^{3}$ Department of Economics and Business Management Sciences, Catholic University of the Sacred Heart, Milan, Italy

Background: To the best of our knowledge, this is the first time that the role of touch has been explored through a neuroscientific approach during an in-store consumer experience. Aims: This research examines the presence of distinct cortical brain oscillations in the brain of consumers while exploring a store, which offers a high degree of sensory stimulation, and using haptics (being allowed to touch or not to touch products). Methods: A 16-channel wireless electroencephalogram (EEG) was applied to 23 healthy participants (Mean age $=24.57 \mathrm{y}$; Standard Deviation $=3.54$ ), with an interest in cosmetics but naïve about the specific store explored. Subjects were assigned to two experimental conditions based on the chance of touching only or not touching the products. Cortical oscillations were explored by means of power spectral analysis of the following frequency bands: delta, theta, alpha, beta. Results: Findings underlined the presence of delta, theta, and beta bands within the frontal brain regions distinctly during these two sensory conditions. The absence of touch was marked by the presence of low-frequency bands (delta and theta) in the left hemisphere, compared to the non-touch condition and this result interpreted as a lack of perceptual experience that needs cognitive control. Whereas a significant presence of beta band characterized the touch compared to non-touch condition and it was associated with the sustained awareness of the sensory experience. Conclusion: Overall, EEG cortical oscillations functional interpretation could help to understand the neurophysiological implicit responses to tactile conditions and the significance of touch integration in consumers' experience.

\section{POSTERS: Submission 8}

\section{The Relation of Mental Rotation and Postural Stability}

\section{Philipp Hofmann, Petra Jansen}

\section{University of Regensburg, Faculty of Human Science,} Germany

Background: Easy and everyday motor tasks, such as standing upright, can be influenced by cognitive tasks. Moreover, there is a connection between various motor processes and mental rotation ability, as a cognitive task. Aims: Main goal of this study was to investigate the influence of different mental rotation tasks on postural stability. Methods: Eighty-four participants were tested with two object-based mental rotation tasks (cube vs. human figures), an egocentric mental rotation task with one human figure, a math- (cognitive control) and a neutral task, while standing on a force plate in a bothlegged narrow stance. Parameters related to the Center of Pressure course over time were used to quantify postural stability. Results: The simultaneous solution of mental rotation tasks has led to postural stabilisation compared to the neutral condition. Egocentric tasks provoked more postural stability than object-based tasks with cube figures. Furthermore, a more stable stance was observed for embodied stimuli than for cube figures. An explorative approach showed the tendency that higher rotation angles of the object-based mental rotation task stimuli lead to more postural sway. Conclusion: These results contribute to a better understanding of the interaction between mental rotation and motor skills and emphasize the role of type of task and embodiment in dual task research.

\section{POSTERS: Submission 9}

Role of Visuospatial and Motor abilities for predicting Geometry in 2-3 graders

Laura María Fernández-Méndez ${ }^{1}$, Chiara Meneghetti ${ }^{2}$, Agustín Martínez-Molina ${ }^{3}$, Irene Mammarella ${ }^{4}$, Mariá José Contreras ${ }^{5}$

${ }^{1}$ Department of Psychology, University Rey Juan Carlos, Spain

${ }^{2}$ Department of General Psychology, University of Padova, Italy

${ }^{3}$ Department Social Psychology and Methodology, University Autónoma of Madrid, Spain

${ }^{4}$ Department of Developmental and Social Psychology, University of Padova, Italy

${ }^{5}$ Department of Basic Psychology I, UNED, Spain

Background: Geometry is a subject frequently associated with maths performance or science interest, as well as reasoning and spatial skills. In school context, Geometry achievement has been connected with visuospatial abilities but less frequently with motor skills, where the embodied cognition approach seems especially important to explain the emergence of complex cognitive representations based in motor processes. Aims: Few studies have assessed previously the contribution of both spatial and motor abilities to predict geometry performance. Thus, in this study, we aimed to examine the role of visuospatial and motor skills in Geometry achievement in primary schoolers. Methods: 215 primary school children (Grades 2 and 3; $51 \%$ boys) were presented with tasks related to visuospatial abilities (Visualization and Mental Rotation), Motor skills (Manual Dexterity, Balance and Throwing and Hold), Fluid Intelligence, Working Memory and Geometry achievement. Results: Working memory, Fluid intelligence, Visuospatial abilities and Motor skills were considered predictors and Geometry achievement was the criterial variable. A multiple linear regression model showed that Geometry was explained (22\% of variance) by Age, Mental Rotation and Manual Dexterity. Furthermore, the regression weights showed that Visualization was relevant to predict Grab-Throw (.285) and Manual Dexterity (.211). Manual Dexterity was significant to predict Geometry (.252). Finally, Mental Rotation was relevant too, for predicting Geometry (.203). Conclusion: The results highlighted the importance of considering both visuospatial factors as well as motor ones, to explain part of the achievement in Geometry in primary schoolers, strengthening the embodied cognition perspective in this developmental stage.

\section{POSTERS: Submission 11}

\section{Cognitive processing during a green hotel exploration:} EEG evidence

Giulia Fronda ${ }^{1,2}$, Federico Cassioli ${ }^{1,2}$,

Roberta Sebastiani ${ }^{3}$, Michela Balconi ${ }^{1,2}$

${ }^{1}$ International Research Center for Cognitive Applied Neuroscience (IrcCAN), Catholic University of the Sacred Heart, Milan, Italy

${ }^{2}$ Research Unit in Affective and Social Neuroscience, Department of Psychology, Catholic University of the Sacred Heart, Milan, Italy 
${ }^{3}$ Department of Economics and Business Management Sciences, Catholic University of the Sacred Heart, Milan, Italy

Background: In the last years, marketing has adopted new sustainable market strategies to limit the adverse effects of products and environments improving individuals' living conditions and environmental well-being. The effects of adopting these new approaches have recently been investigated thanks to neuroscience's contribution able to provide information about cognitive and emotional mechanisms related to consumers' behaviors. Aims: In order to investigate consumer behavior towards the adoption of a sustainable approach in tourism marketing, a neuroscientific paradigm, consisting in the use of electroencephalography (EEG) to record individuals' neural responses, has been implemented to understand individuals' cognitive processes during the exploration of a green hotel. Methods: In particular, neural correlates of a sample of 19 healthy subjects were collected through the use of EEG, for the recording of fronto-central and temporo-parietal activity. Specifically, participants were asked to explore four different areas of the hotel (restaurant, bedroom, hall, and bar) composed of eco-friendly elements. Results: The results of the study revealed a different cortical activation concerning the activity of EEG alpha, beta, delta, and theta frequency bands during the exploration of the green hotel spaces, with an increase in temporoparietal beta and theta activity. This result underlines an increase in individuals' attentional and cognitive processing and emotional engagement during the exploration of the environment. Conclusion: These results allow us to underline how the application of a neuroscientific paradigm is useful for investigating individuals' attitudes and preferences to design spaces that are both ergonomic and comfortable but attentive to the issue of sustainability.

\section{POSTERS: Submission 13}

\section{Visual representation as a link between manual and mental rotation}

\section{Leonardo Jost, Petra Jansen}

\section{University of Regensburg, Germany}

Background: Studies have demonstrated a common process between manual rotation (the rotation of an object by rotary hand movements) and mental rotation (the rotation of an object in the mind). Training studies have shown that a manual and concurrent visual rotation improves mental rotation performance. Aims: Due to the lack of separation of the visual and motor component in manual rotation trainings, it is the main goal to determine which component of the manual rotation training influences mental rotation performance. Methods: A total of 121 participants were randomly assigned to visual training, manual rotation training using a steering wheel, or manual training without rotary movement. Before and after the training session of 30 minutes, they had to solve a chronometric mental rotation test. Results: Data showed an improvement in mental rotation performance for all groups. However, this improvement did not differ between groups. Conclusion: Due to the independence of the form and occurrence of the manual activity, this suggests that it is not the motor activity but the concurrent visual rotation that leads to improvements in mental rotation tasks. Therefore, the visual component in mental rotation tasks has to be investigated in more detail.

\section{POSTERS: Submission 22}

Orienteering practice: how does it relate to visuospatial individual factors?

\section{Chiara Meneghetti, Tommaso Feraco, Marco Bonvento}

Department of General Psychology, University of Padova, Italy

Background and Aim: Orienteering is a sport that involves navigating in the environment. As the ability to navigate is associated with individual visuospatial factors (e.g. Hegarty et al. 2006; Pazzaglia et al. 2018), it is worth examining how orienteering practice relates to people's visuospatial abilities, wayfinding attitudes, everyday spatial habits, and environment knowledge acquisition (e.g. from a map). Methods: Our study sample consisted of three groups: 17 orienteering experts (E), and 17 beginners (B), selected by their years of practice and number of competitions entered, and 17 controls (C) engaging in physical activities for leisure. Participants completed visuospatial cognitive tasks, answered questionnaires on their wayfinding attitudes and everyday spatial habits, and were assessed on their recall of information learned from a map. Results: A Bayesian analysis showed that group E (compared with group C): (1) scored higher in spatial visualization and rotation tasks; (2) tended more to report a good sense of direction, and a knowledge and use of cardinal points; (3) preferred to use maps in everyday displacements; and (4) recalled information learned from a map more accurately. For some measures, group E fared better than group B, and group B fared better than group C. Conclusion: Our results point to the benefits of orienteering practice on visuospatial abilities, wayfinding attitudes (especially as concerns extrinsic frames of reference), spatial habits, and learning from a map. These results contribute to clarifying the association between navigation experience gained by orienteering and individual visuospatial factors.

\section{POSTERS: Submission 30}

\section{Subjective Time Perception in Musical Imagery: An fMRI Study on Musicians}

\section{Morteza Izadifar \\ Institute of Medical Psychology, Ludwig-Maximilian University Munich, Munich, Germany}

Perception of time-the way we subjectively experience time, what time feels like to us as individuals - is a boundlessly interesting topic because time persistently surprises us; a good holiday races by, yet the moment you arrive home, it feels as though you have been away centuries. How do we interpret the length of time? How is the subjective time perception in imagery? The cognitive preparation of an operation without overt motor execution is referred to as imagery (of any kind). Over the last two decades of progress in brain timing studies, the timing of imagery has received less focus. This study compared the time perception of 7 professional violists' actual and imagery performances to see if such an analysis could offer a different model of timing in musicians' imagery skills. When comparing the timing profiles of the musicians between the two situations (actual and imagery), we found a significant correlation in overestimation of time in the imagery. In our fMRI analysis, we found high activation in the left cerebellum. This finding seems close to dedicated models of timing such as the cerebellar timing hypothesis which assigns a 'specialized clock' for the tasks. 


\section{POSTERS: Submission 34}

\section{Analysis of spatial behaviour by the means of Euclidean} Distances.

Antonietta Argiuolo ${ }^{l}$, Federica Somma ${ }^{l}$, Davide Marocco ${ }^{l}$, Onofrio Gigliotta ${ }^{1}$, Paolo Bartolomeo ${ }^{2}$, Orazio Miglino ${ }^{1}$, Michela Ponticorvo ${ }^{1}$

${ }^{1}$ University of Naples Federico II Natural and Artificial Cognition Lab, Italy

${ }^{2}$ French Institute of Health and Medical Research Inserm, France

Background: Spatial behaviour can be analysed using numerous indexes that vary depending on the task and on the variables involved. Starting from widely employed task for spatial attention, called BTT and its enhanced version E-BTT (administered through platform E-TAN), we propose using two indexes based on Euclidean Distances. Aims: Here, we show how to exploit Euclidean Distances to identify similar strategies to solve a task and to evaluate them. Methods: E-BTT through E-TAN was administered to a sample of 100 undergraduate students. The task asked to place 16 disks as evenly as possible within the surface area surrounded by a wooden frame. A 30fps camera placed above the table detected them and estimated 16 pairs of coordinates. To define indexes to discriminate behavioural strategies, the following Euclidean Distances were calculated: (1) Between Distances (BDs), which measure the distance between each pair of participants' corresponding disks; (2) Within Distances (WDs), calculated by adding the distance between consequently placed disks. Results: BDs for all participants were organized in a matrix with $\operatorname{Min}=30.41 \mathrm{~cm}$ and $\operatorname{Max}=693.28 \mathrm{~cm}$. Low distances (D1) were used as a threshold to identify similar strategies. Considering WDs, the average was $261.04 \mathrm{~cm}$ (DS = 88.86); organized and disorganized sequences resulted different in terms of WD $(\mathrm{t}(95)=2.52 ; p<0.05)$, with organized sequences having lower average. Conclusion: Euclidean distances between two different sequences helped grouping similar strategies, whereas Within Distance allows to distinguish between organized and disorganized spatial strategies. These indexes can be usefully employed in spatial behaviour analysis.

\section{POSTERS: Submission 36}

\section{Early development of visuo-spatial ability and correlations with ADHD}

\section{Enikö Györkö, Gabriella Király}

University of Pécs Faculty of Cultural Sciences, Education and regional Development, Hungary

Background: Attention-Deficit/Hyperactivity Disorder (ADHD) is impressing to deficits in cognitive functions, thereby affecting development of Visuo-Spatial Abilities (VSA). The VSA plays a crucial role in visual information processing, visual-spatial imagination, visual-spatial working memory, perceptual processing, visuomotor coordination. Aims: The first aim was to explore different VSA factors in 22 preschool-age (6-7 years) children with ADHD and explore early atypical VSA markers in ADHD. The second objective of the study was to explore the Processing Speed factors' ability to predict the performance of ADHD participants in VSA, which is a key factor in learning success. Methods: We measured VSA through WISC IV intelligence test, from which we used perceptual reasoning index (PRI) and subscales furthermore, processing speed index (PSI) and subscales. Results: Participants with ADHD showed weaker performance in PRI, PSI and Block Design contrary to normal controls. Regression analysis exhibited PSI were able to predict response PRI, Block Design of subscale and Matrix Reasoning of subscale. Conclusion: Our study adds to this research field results in favor of the existence of alterations in the visuo-spatial development of children with ADHD. Regarding these results, we consider it important to involve targeted therapeutic assistance for these factors before school age, for preschool children with ADHD.

\section{POSTERS: Submission 38}

\section{Separating sagittal and transverse in egocentric frame of reference}

\section{Bill Palmer}

\section{University of Newcastle, Australia}

The standard typology of the egocentric ("relative") frame of reference (FoR) treats the sagittal (front-back) and transverse (left-right) axes as a unitary system, interacting in three strategies: reflectional, rotational, and translational (Hill 1982; Levinson 2003). This typology is inadequate: Treating sagittal and transverse as a unitary system fails to recognize commonalities cross-cutting the three subtypes (e.g. sagittal is the same in reflectional and rotational). And it does not allow for systems where only one axis is encoded, and therefore cannot type such systems (e.g. systems encoding only sagittal may conform to both reflectional or rotational). This sometimes leads to an erroneous claim that a particular language lacks egocentric FoR entirely, based on an absence of transverse encoding, when egocentric encodings are in fact present on the sagittal. A new approach outlined here resolves these inadequacies by typologizing sagittal and transverse independently. In this approach, sagittal has two orientation strategies: 'facing' ("front" side closest to speaker) and 'aligned' ("front" side away from speaker). Transverse also has two strategies: 'aligned' ("left" and "right" sides correspond to speaker's left-right) and 'rotated' ("left" and "right" are reversed). The traditional types fall out of varying interactions of the independent sagittal and transverse axes. For example, the reflectional strategy familiar from English combines a facing sagittal axis with an aligned transverse. By treating sagittal and transverse independently, this new typology generates all egocentric FoR types, while accounting for previously obscured facts about the nature, distribution and use of egocentric FoR.

\section{POSTERS: Submission 41}

Agoraphobia disorder and spatial working memory

Micaela Maria Zucchelli ${ }^{1}$, Laura Piccardi ${ }^{2,3}$, Raffaella Nori ${ }^{l}$

${ }^{1}$ Department of Psychology, University of Bologna, Italy ${ }^{2}$ Department of Psychology, La Sapienza University of Rome, Italy

${ }^{3}$ Cognitive and Motor Rehabilitation and Neuroimaging Unit, IRCCS Fondazione Santa Lucia, Italy

Background: Individuals with agoraphobia, who have fear of moving in large or crowded open spaces, exhibit an impaired exploratory activity when navigating in an unfamiliar environment. However, prior studies poorly investigated their difficulties in processing spatial information. Aims: The aim of study was to investigate the contribution of spatial working memory (SWM) in the ability to acquire and 
process spatial information in individuals with agoraphobia, considering the use of egocentric and allocentric coordinates, and environments with or without people. Methods: A total of 106 individuals (53 with agoraphobia-53 controls) navigated in a virtual public square to acquire spatial information which included recognition of landmarks, the relationship between landmarks and themselves (egocentric coordinates) and regardless themselves (allocentric coordinates). Half of both groups navigated in a square without people and the other in a crowded one. They completed forward and backward Corsi test to measure spatial working memory (requiring to mimic a spatial sequence of blocks in the same or reversed order) and 3 tasks measuring landmark recognition, egocentric and allocentric judgments concerning the explored square. Results: Individuals with agoraphobia exhibited a reduced SWM only in backward version of Corsi test (spatial sequence in reversed order), a worse performance in landmarks recognition only in the crowded condition, and in both conditions as regards egocentric and allocentric tasks. Moreover, a defective SWM predicted a worst performance in egocentric and allocentric tasks. Conclusion: Results suggested that individuals with agoraphobia exhibit spatial difficulties particularly when performing complex spatial tasks requiring them to process and transform information simultaneously.

\section{POSTERS: Submission 50}

\section{Action and defence function of the reaching space: effect of humans and objects}

\section{Antonella Ferrara, Tina Iachini, Gennaro Ruggiero}

Laboratory of Cognitive Science and Immersive Virtual Reality, CS-IVR, Department of Psychology, University of Campania Luigi Vanvitelli, Italy

Background: We constantly monitor the valence of stimuli entering the space near our body, both humans and objects. This area represents a space for action on objects that can be defined as the reaching space. Importantly, it is also conceived as a protective buffer against threats. This defensive function allows us to act effectively and safely to protect our body. However, it is still unclear whether the reaching space around the body reflects a similar modulation in the presence of objects or social stimuli with emotional valence. Aims: We aim to understand whether the reaching space is modulated similarly or differently by the intrusions of conspecifics versus non-social virtual stimuli, both with positive and negative valence. Methods: In IVR, participants were asked to provide reaching-distance judgements (i.e., judge whether or not a stimulus is reachable) while being approached by virtual humans showing positive or negative facial expressions and virtual objects with threatening or non-threatening value. Results: The results showed that the overall reaching distance was larger with negative than positive virtual stimuli. However, there was no significant difference between humans and virtual objects when they expressed a positive valence. Instead, when stimuli expressed a negative valence there was a larger widening of the distance with objects than humans. Conclusion: These results confirm the defensive function of reaching space encoding, which is reflected in a large margin of safety from approaching threats. However, the clear effect of dangerous objects over threatening people may reflect the primacy of defending our physical integrity.

\section{POSTERS: Submission 55}

A co-bot quality cycle: neuroscientific view on fatigue and executive functions

Federico Cassioli ${ }^{1,2}$, Giulia Fronda ${ }^{1,2}$, Michela Balconi ${ }^{1,2}$

${ }^{1}$ International Research Center for Cognitive Applied Neuroscience (IrcCAN), Università Cattolica del Sacro Cuore di Milano, Italy

${ }^{2}$ Research Unit in Affective and Social Neuroscience, Department of Psychology, Università Cattolica del Sacro Cuore di Milano, Italy

Background: Industry 4.0 aims at developing collaborative robotic technology (co-bot) to improve efficiency, ergonomics and safety in the workplace. Aims: The aim of this contribution was to propose a quality-cycle to optimize human robot interaction (HRI) for co-bot technology and highlighting if the perceived cognitive effort and spatial perception change in HRI compared to human-to-human interactions. Methods: Based on the Deming quality-cycle and the concept of neuro-industrial engineering, we selected the following neuroscientific dimensions which should be integrated in the quality cycle for co-bot designing: fatigue, executive functions, attentional coordination, selective attention and space perception. Regarding the latter, we propose the consideration of peripersonal and extrapersonal dimensions. Results: The outcome is a four-phases cycle composed by the first step (planning) where a compartmentalization of the industrial processes is made and the brain-computer interface and neuroscientific methods are selected based on the type of HRI [e.g. to assess cognitive and emotional planning, $(\alpha / \theta) / \beta$ or $(\alpha / \theta) /(\alpha+\beta)$ ratios in the frontal and central brain can be selected]. In the second phase (doing) virtual/real scenarios are executed while data is retrieved. In the third phase (modelling) data are used to create bottom-up models, which will be tested again in the future. Finally, in the last phase (change), evidence-based adjustments are implemented. Conclusion: An integrated perspective, which considers the worker from a holistic viewpoint, as the one presented, might make co-bots more humanoriented, leading to increased efficiency and safety by using data on human perception and spatial representation.

\section{POSTERS: Submission 56}

\section{Impacts of COVID-19 control measures} on metacognition and spatial cognition

\author{
Roland Kasek ${ }^{1}$, Szabolcs Takács ${ }^{2}$, Aniko Kónya ${ }^{3}$, \\ Imre Lázár ${ }^{l}$ \\ ${ }^{1}$ Institute of Behavioural Sciences, Semmelweis University, \\ Budapest, Hungary \\ ${ }^{2}$ Institute of Psychology, Karoli University of the Reformed \\ Church, Budapest, Hungary \\ ${ }^{3}$ Department of Cognitive Science, Institute of Psychology, \\ Eötvös Loránd University, Budapest, Hungary
}

Background: The negative effects of psychosocial discomfort and isolation on cognitive performance are prevalent. Metacognitive abilities - that also play a major role in human social interactionsrequire sufficient excess of mental capacity, hence more vulnerable to constant distress and emotional load. Aims: The study investigated how COVID-19 control measures affected basic spatial cognition and metacognitive abilities compared to data collected in the previous years. Methods: The repeated cross-sectional research (2014-2020) 
examines a sample of healthy adult subjects (sine morbo, $18-72, \mathrm{~N}=$ 857 ) in four immediate spatial manipulation tasks of increasing complexity (3-9 span and preset exposition time) and tested online. Task 1 is a digital version of Corsi (CBTT) and baselines for correct localisation. Task 2 and 3 demands temporal conversion of numeric information, while Task 4 requires temporal-numeric interference inhibition. Metacognitive factors are measured in self-pacing (Task 2-3 difference) and accuracy of self-peer performance comparison. Results: COVID-19 control measures have significant negative effect on metacognitive ability $(\mathrm{F}=1.525 ; \mathrm{df}=65 ; \mathrm{p}=0.006)$ and learning new tasks $(\mathrm{F}=1.550 ; \mathrm{df}=65 ; \mathrm{p}=0.005)$ compared to previous years with the interaction of age. Accuracy of self-peer performance comparison seems to be intact in contrast to pre-covid times. Conclusion: Annual comparison of spatial recall and metacognitive performance indices show that COVID-19 control measures affected older people more than young ones, independent of gender. Further investigation of how quickly subjects mentally adapt to existential threat and cope with psychosocial discomfort may reveal important findings for the development of diagnostic methods and therapeutic interventions.

\section{POSTERS: Submission 63}

\section{Neural correlates of spatial reorientation and egocentric localization in memory}

\section{Léo Dutriaux, Yangwen Xu, Nicola Sartorato, Roberto Bottini}

\section{University of Trento, Italy}

In a well-known environment, humans are able to remember the egocentric location of the different objects it contains. For instance, while seated in the middle of the bedroom, one can retrieve the location of their bed relative to their current heading. The neural bases of such an egocentric spatial process are still uncertain. This study aimed to shed light on this question using fMRI and Representational Similarity Analysis (RSA). Before the fMRI session, participants were trained to remember the location of objects within different rectangular rooms respecting the same spatial geometry. During the fMRI session, each trial started with the presentation of a geometrical map that could represent any room, and participants were instructed to imagine themselves facing one of the walls. Second, they were presented with an object that they had to locate egocentrically relative to their imagined heading. The RSA showed that heading direction was represented in the occipital place area (OPA), while the egocentric position of the objects was represented in the parietal cortex (the left angular gyrus and bilateral precuneus). The OPA is known for its role in the perception of scenes and is thought to allow visually guided navigation by representing environmental boundaries. However, these results indicate newly that the OPA is also involved in memory-guided navigation. It also suggests that it represents environmental boundaries, to some extent, abstracted from perception. Overall, these results suggest the boundary represented in OPA served as a reference to the parietal cortex to compute the egocentric location of the object.

\section{POSTERS: Submission 64}

\section{Audio-Tactile dynamic multisensory integration} in sighted and blind individuals

\author{
Maria Casado Palacios ${ }^{1,2}$, Monica Gori ${ }^{1}$,
} Claudio Campus ${ }^{1}$

\section{${ }^{1}$ Istituto Italiano di Tecnologia, Italy}

${ }^{2}$ University of Genoa, Italy

Our performance is typically enhanced when the information comes from multiple senses compared to a single one. However, after a sensory deprivation, the cortex experiences several changes, leading to a different performance of those with sensory impairment than typical individuals. In this regard, not much is known about the mechanisms underlying auditory-tactile integration, especially in blind people. Here, we investigated the role of tactile input in the tactile-auditory interaction. Our study measure the velocity discrimination threshold of 15 blindfolded sighted and 15 blind participants. The dynamic tactile stimuli were provided by physical wheels with a sinusoidal profile, while the audio signal was generated by an audio device aligned with the tactile stimulation. The participant had to perceive a sequence of two movements with different speeds and discriminate the faster between the two. In three experimental sessions, the participant could either touch (unimodal tactile), hear (unimodal audio), or both (bimodal audio-tactile) the dynamic stimuli. A total of 180 trials were collected (60 per condition). Our results show an improvement in bimodal conditions compared to the unisensory ones. Interestingly the same benefit was not evident in people with visual impairment. These results suggest that audio-tactile information of dynamic stimuli is better integrated in sighted than in blind individuals. These results support the idea that the visual modality is essential for developing spatial processing such as speed discrimination. A visual calibration process might mediate this process over the other audio and tactile sensory modalities.

\section{POSTERS: Submission 67}

\section{Movement and Haptic Anticipation: clues to Facilitate} Human-Robot Interaction?

Arthur-Henri Michalland ${ }^{1,2,3}$, Benjamin Navarro ${ }^{2}$, Philippe Servajean $^{3}$, Denis Brouillet ${ }^{3}$, Philippe Fraisse ${ }^{2}$

${ }^{1}$ Université Montpellier-LIFAM, France

${ }^{2}$ CNRS-UM, LIRMM, Interactive Digital Humans, France

${ }^{3}$ Univ Paul Valéry Montpellier 3, EPSYLON EA 4556, Montpellier, France

As an object's weight impacts the forces necessary to adapt to its dynamical properties (Johansson, Häger, Riso 1992), it needs to be anticipated before grasping and manipulation. Two phenomena modulate those forces: anticipation of the object's haptic properties and haptic feedback. Our aim was to assess the relations between these phenomena in object's displacement, and evaluate a possible use in human-robot interactions. In the first part of this study participants displaced five visually identical objects with weights varying from 0.220 to $2.220 \mathrm{Kg}$. Trajectories of their hand was recorded with a sensor suit while their knowledge of the object's weight was manipulated. Two criteria were used to study the trajectories: a criterion of trajectory deviation from individualized reference trajectories, and the mean jerk. Overall we found that participants performed stable trajectories for a given weight, even if they showed variations in the jerks at the beginning of their movement depending on their knowledge of the object's properties. In other words, 
participants did not used solely haptic anticipation and feedback, but also the uncertainty associated with their anticipation, reflected in distinct motor strategies. In the second part, an open-loop torquecontrolled robotic manipulator simulated the participants' trajectories in similar experimental conditions to evaluate deviations when no feedback was given. A methodology is then suggested to compare Human and Robotic trajectories for setting feedback delays that may enhance human-robot interaction (e.g., hand-over). Those variable delays would allow deviations from optimal trajectories, giving clues about objects properties (e.g., heavier or lighter than expected) to human collaborators.

\section{POSTERS: Submission 74}

\section{Effects of complexity and naturalness of pocket park designs on user preference}

\author{
Guzin Oztok ${ }^{1}$, Doga Gulhan ${ }^{2}$ \\ ${ }^{1}$ Independent researcher, currently not affiliated \\ ${ }^{2}$ Royal Holloway, University of London, United Kingdom
}

Previous research on landscape preference has been investigating the effects of the natural or built environment on user behaviour and preference, often either as studies on large-scale green spaces or qualitative case studies. However, empirical research on small-scale public green spaces, particularly with design concepts as operationalized variables, is very limited. This presented research aimed to measure user preference for various spatial features of a pocket park, and also to demonstrate a scalable method using a workflow of parametrically modified designs. Here, digitally rendered design variations of a pocket park were generated by manipulating levels of complexity and naturalness (as two frequently used design concepts), and participants' judgments on these variations were recorded. The preliminary results with a sample of 75 participants in an online experiment showed that (1) positive linear correlations were observed between liking and perceived complexity, and between liking and perceived naturalness, (2) liking was more prone to change by the complexity level, compared to the naturalness level, (3) the design variation with high complexity and high naturalness was preferred most, and (4) further insights were gained from the supplementary questionnaire about user attitudes. Now, the research is being expanded into a virtual reality (VR) experiment by using 3D pocket parks and by creating more nuanced levels of design attributes. Overall, these initial results showed the feasibility of the proposed method for measuring user judgments in a controlled environment. This can be seen as a promising method for empirical research and the co-creation of public green spaces.

\section{POSTERS: Submission 77}

\section{Space for relax, spaces for rechange: effect of urban spaces on people's mood}

\author{
Mariachiara Rapuano ${ }^{1}$, Tina Iachini ${ }^{1}$, Gennaro Ruggiero ${ }^{1}$, \\ Massimiliano Masullo ${ }^{2}$, Luigi Maffei ${ }^{2}$, Alice Palmieri ${ }^{2}$, \\ Francesco Ruotolo \\ ${ }^{1}$ Laboratory of Cognitive Science and Immersive Virtual \\ Reality, Department of Psychology, University of Campa- \\ nia Luigi Vanvitelli, Italy \\ ${ }^{2}$ SENS i-Lab, Department of Architecture and Industrial \\ Design, University of Campania Luigi Vanvitelli, Italy
}

Background: In recent years, urban planners and architects have tried to relate the design of new urban spaces to users' emotions (Ellard 2020; Spence 2020). It is well known that green parks characterized by naturalistic elements have a positive impact on people's mood (Kaplan 2001). However, urban living often limits access to nature and can increase stress and reduce psychological well-being (WHO 2016). Aims: This study aimed to identify features of urban parks other than greenery that can have a positive impact on different aspects of people's mood, such as calmness, energy, and happiness. Methods: Participants were presented with 15 images depicting three categories of urban parks, i.e. Green Parks with only greenery and water, Coloured Parks with naturalistic and colourful architectural elements, and Squares with street furniture and some green elements. Participants had to rate on a 9-point Likert scale how much each image induced calmness, energy, happiness, nervousness, tiredness and sadness $(0=$ not at all; $9=$ very much $)$. Results: In line with the literature, green spaces had a more positive impact on participants' mood than Colored Parks and Squares. However, Colored Parks, as compared to Squares, were considered to be more energizing and made people feel less sad. Finally, while green spaces were rated as more calming than energizing, the opposite was true for Colored Parks. Conclusion: Different types of urban spaces can positively affect different mood dimensions, from relaxation in Green Spaces to recharging in Colored Spaces.

\section{POSTERS: Submission 79}

\section{The influence of sounds on individual's mood}

Tina Iachini ${ }^{l}$, Mariachiara Rapuano ${ }^{1}$, Gennaro Ruggiero ${ }^{1}$, Francesco Ruotolo ${ }^{1}$,Federico Cioffi ${ }^{2}$, Luigi Maffei ${ }^{2}$, Massimiliano Masullo ${ }^{2}$

${ }^{1}$ Laboratory of Cognitive Science and Immersive Virtual Reality, Department of Psychology, University of Campania Luigi Vanvitelli, Italy

${ }^{2}$ SENS i-Lab, Department of Architecture and Industrial Design, University of Campania Luigi Vanvitelli, Italy

Background: How do sounds influence people's moods? Answering this question is crucial for urban planners to design urban spaces that, not only visually, but also acoustically have a positive impact on users (Atkinson 2007). Several studies have shown that natural sounds have a relaxing effect on people (Benfield et al. 2014). However, individuals may use urban spaces to relax and recharge or to remedy a sad mood. Aims: This work aimed to assess the influence of different types of sounds on different mood dimensions. Methods: Participants listened to seven types of sounds: natural (i.e. wind, water), animals (i.e. horses, birds), anthropic (i.e. adult and child voices), manmade (i.e. bells, traffic), classical music (i.e. piano, violin), percussion (i.e. drums, bongos), and electronic music. Participants had to rate on a 9-point Likert scale how much each sound induced calmness, energy, happiness, nervousness, tiredness and sadness $(0=$ not at all; $9=$ very much). Results: The most calming sounds were the natural sounds and classical music, followed by animals. However, classical music also made participants sadder than all other sounds. On the other hand, percussion induced more energy, while natural and animal sounds induced more happiness than other sounds. Finally, electronic music made people more energetic and happier than anthropic and manmade sounds. The latter made participants more nervous. Conclusion: Different types of sound can influence mood dimensions differently. Therefore, a sound space could be designed according to the dimensions of mood it aimed to promote. 


\section{POSTERS: Submission 81}

\section{Effects of different kind of sports on (perceived) mental rotation performance}

\section{Martina Rahe}

Institute of Psychology University of Koblenz-Landau, Germany

Background: Men usually outperform women in mental rotation and rate their performance higher. Positive effects of some kind of sports on mental rotation are also well documented. Aims: Effects of participants' sex and their practised sport on their mental rotation performance and their perceived mental rotation performance should be analysed as well as interaction effects of sex and sports. Methods: Participants were 71 people (39 men) between 18 and 42 years $(\mathrm{M}=$ $24.79, \mathrm{SD}=4.52)$. Participants were categorized due to their practised kind of sport (sports that could/could not promote spatial abilities). All participants solved a mental rotation test and filled out a questionnaire about their perceived mental rotation performance (PMR), their age, sex, and practise sports. A sum score of correct items in the mental rotation test (MR) and the percentage of correct items on tried items (\%MR) were calculated. Results: Main effects of sex showed higher scores of MR $\left(\right.$ eta $\left.^{2}=.170\right), \%$ MR $\left(\right.$ eta $\left.^{2}=.097\right)$, and PMR $\left(\right.$ eta $\left.^{2}=.228\right)$ in men than in women and higher scores of PMR $\left(\mathrm{eta}^{2}=.060\right)$ in participants who practised sports that could enhance spatial abilities. Significant interaction effects of sex and sports on \%MR (eta $\left.{ }^{2}=.099\right)$ and on PMR $\left(\mathrm{eta}^{2}=.062\right)$ showed higher effects of beneficial sports in women than in men. Conclusion: Beneficial kind of sports can have positive effects on (perceived) mental rotation performance especially in women. That could be used to promote spatial abilities in girls.

\section{POSTERS: Submission 97}

\section{Egocentric, Axial Information in Allocentric Representations in aṣ-Ṣānị Arabic}

\section{Letizia Cerqueglini}

\section{Tel Aviv University, Department of Hebrew Language and} Semitic Linguistics, Lecturer, Israel

Linguistic and cognitive spatial frames of reference (FoRs), used to project coordinates onto spatial arrays to locate an object (Figure, F) in relation to another object (Ground, G), are egocentric (depending on the coordinates of the observer, $\mathrm{O}$ ) or allocentric (not depending on O). Allocentric FoRs may be intrinsic (depending on the G's inherent sides) or geocentric (depending on external sources) (Bohmeneyer \& O'Meara 2012). Twenty Traditional as-SāniC Arabic speakers (TAA; Negev Arabic) were tested on "Man \& Tree" (Levinson et al. 1992) and "Ball \& Chair" (Bohnemeyer 2008). Given TAA giddām, 'in front of' (only intrinsic) and šarg 'east' (geocentric), F min G w-giddām/šarg, ' $\mathrm{F}$ is from $\mathrm{G}$ and in front/east' means that $\mathrm{O}$ is aligned with FG along the same cardinal axis; $F$ min giddām/šarg $G$, ' $F$ is from in front/east of $\mathrm{G}^{\prime}$ means that $\mathrm{O}$ is not aligned with $\mathrm{FG}$; and $\mathrm{F}$ giddām/šarg G, 'F is in front/ east of G' is unmarked in relation to O's position. Recall and memory tasks (Levinson 2003) proved TAA informants' sensitivity to OFG axial alignment. TAA allocentric strategies can encode O's position vis-à-vis FG. This is unprecedented in the literature, similar only to Gawwada (Cushitic), which distinguishes geocentric representations in which $\mathrm{O}$ is/is not between FG along the same cardinal axis and $\mathrm{O}$ is not on the same cardinal axis as FG (Tosco 2006). Hausa (Chadic) uses egocentric strategies with OFG aligned (Hill 1982), so the attention to OFG mutual, axial alignment may represent a pan-Afroasiatic heritage.

\section{POSTERS: Submission 108}

How to measure writing and drawing tilts after stroke: methodological approach

Flora Diaine, Rémi Lafitte, Olivier Carré, Shenhao Dai, Anaïs Verdier, Céline Piscicelli, Dominic Pérennou

Laboratoire de psychologie et neurocognition CNRS UMR 5105, Grenoble-Alpes université, clinique de médecine physique et réadaptation, CHU de Grenoble-Alpes, 38034 Grenoble, France

Backgrounds: Tilted writing and drawing are frequent after righthemisphere stroke, and should be more systematically quantified. Aims: To implement valid and reliable tools and criteria to quantify and diagnose post-stroke tilted writing and drawing, determinants of which are analysed in the companion abstract 110. Methods: We asked 130 individuals to write their name and address and to copy the Gainotti scene: 69 after a RHS (1-3 months), and 61 matched healthy controls. Two criteria were measured for writing (mean lines' horizontal tilt and margin's vertical tilt) and drawing (mean baselines' horizontal tilt and mean vertical tilt of the two central trees), by two independent evaluators. Inclinations were measured by a software from specific landmarks positioned by each evaluator. Inter-rater reliability and accuracy were determined by the intraclass correlation coefficient (ICC), standard error of measurement (SEM), and smallest detectable change $\left(\mathrm{SDC}_{95}\right)$. Normality was mean $\pm 2 \mathrm{SD}$ from controls' data. Results: ICC were high both in patients and controls (all $>$ .864). SDCs $_{95}$ calculated in patients were very satisfactory for the writing (lines $=1.2^{\circ}$; margin $=2.6^{\circ}$ ) and baselines of the scene $\left(1.8^{\circ}\right.$ ). Counterclockwise tilts defining normality were: lines $=-2.9^{\circ}$, margin $=-4.6^{\circ}$, and baselines $=-1.3^{\circ}$. At least one abnormal criterion was found in 22 patients (32\%) who presented greater counterclockwise writing tilts than controls and other patients (all $p<.01)$. Conclusion: This study gives normality ranges and validates tools and criteria to easily measure the writing and drawing orientation after RHS. This was a prerequisite for analysing their determinants (companion abstract).

\section{POSTERS: Submission 129}

\section{The eccentricity effect on endogenous attention}

\author{
Yu Tong ${ }^{1}$, Yunfei Fan ${ }^{2}$ \\ ${ }^{I}$ Department of Psychology and Beijing Key Laboratory of \\ Behavior and Mental Health, Peking University, PR China \\ ${ }^{2}$ Academy for Advanced Interdisciplinary Studies, Peking \\ University, Beijing, China
}

It has been shown that there is a functional subdivision between the central and peripheral visual field. Previous research on inhibition of return further suggests that attentional modulation in the visual field also underlies a functional subdivision. Specifically, inhibition of return effect was found much larger at the periphery relative to the central visual field with a border of approximately 15 degrees eccentricity. However, previous studies demonstrating the functional dissociation of attentional control in the visual field all focused on exogenous attention effect, whether endogenous attention is also influenced by eccentricities remains unknown. To investigate the effect of stimulus eccentricity on endogenous attention, two experiments with either unpredictive or predictive central cue (a direction word left or right) was adopted in the present study with a spatial cuing paradigm. The targets appeared at either the cued or the uncued 
opposite location at two stimulus eccentricities (5 degrees vs. 20 degrees). The results showed significant cueing effects independent of the cue predictability. Moreover, the cuing effect was significantly larger in the periphery relative to the central visual field in both experiments. These results further suggest a functional dissociation of endogenous attentional control in the perifoveal and peripheral visual field.

\section{POSTERS: Submission 131}

\section{Multiple temporal reference frames: Evidence from a temporal reproduction task}

\author{
Yunfei Fan ${ }^{1}$, Xiaoxuan $\mathrm{Li}^{2}$, Zehao Wang ${ }^{2,3}$ \\ ${ }^{1}$ Academy for Advanced Interdisciplinary Studies, Peking \\ University, Beijing, China \\ ${ }^{2}$ School of Psychological and Cognitive Sciences, Peking \\ University, Beijing, China \\ ${ }^{3}$ School of Mathematical Sciences, Peking University, \\ Beijing, China
}

Temporal perception is not only influenced by the current stimulation, but also the distribution of stimulus durations. By establishing an internal reference based on previous experience, participants could optimize their temporal performance. It has been shown previously that perceived durations are systematically biased towards the mean of the internal reference, resulting in regression to mean. Although a single temporal reference has been demonstrated, whether multiple temporal references can be established and maintained at the same time still remains an open question. The present study aimed to explore whether participants can implicitly learn to establish multiple temporal references in temporal reproduction task with a ready-set-go paradigm, which means that subjects should precept the duration defined by two stimuli and reproduce this duration by press the key after the second stimulus. Two different but overlapping distributions of stimulus intervals (short vs. long) were presented at different spatial locations. Participants were asked to reproduce the durations of the presented stimuli in two different conditions: one being informed, and one being not informed the distribution of stimulus durations. The results showed that the participants were able to learn to set up two separate internal temporal references, no matter they were informed the duration distribution or not. In addition, the temporal reference of the shorter distribution was always attracted by the longer distribution. These findings suggest that multiple temporal reference frames can be established and maintained during temporal reproduction process by explicit or implicit learning.

\section{POSTERS: Submission 133}

\section{On a taxonomy of early visual-spatial abilities in testing} versus training

Anna Meinhardt ${ }^{1,3,4}$, Stefanie Jung ${ }^{1,9}$, David

Bräuning ${ }^{1,5,7,9}$, Tahereh Pazouki ${ }^{6}$, Véronique Cornu ${ }^{6}$, Christine Schiltz ${ }^{8}$, Korbinian Moeller ${ }^{1,2,3,4,9}$

${ }^{1}$ Leibniz-Institut für Wissensmedien, Schleichstraße 6, 72076 Tübingen, Germany

${ }^{2}$ Centre for Mathematical Cognition, School of Science, Loughborough University, Loughborough, United Kingdom
${ }^{3}$ DIPF Leibniz Institute for Research and Information in Education, Rostocker Straße 6, 60323 Frankfurt, Germany ${ }^{4}$ Center for Individual Development and Adaptive Education of Children at Risk (IDeA), Frankfurt, Germany

${ }^{5}$ LEAD Graduate School and Research Network, University of Tübingen, Germany, Tübingen, Germany

${ }^{6}$ Luxembourg Centre for Educational Testing (LUCET), University of Luxembourg, 11, Porte des Sciences, 4366 Esch-sur-Alzette, Luxembourg

${ }^{7}$ Hector Research Institute of Education Sciences and Psychology, University of Tübingen, Germany, Europastraße 6, 72072 Tübingen, Germany

${ }^{8}$ Department of Behavioral and Cognitive Sciences (DBCS), University of Luxembourg, 11, Porte des Sciences, 4366 Esch-sur-Alzette, Luxembourg

${ }^{9}$ University of Tübingen, Germany, Geschwister-SchollPlatz, 72074 Tübingen, Germany

Visual-spatial abilities (VSA) were suggested to develop in childhood along the dimensions of intrinsic vs. extrinsic and static vs. dynamic characteristics (Newcombe \& Shipley 2015). The validity of this taxonomy was evaluated recently for testing situations, using various tasks for each of the four categories (Mix et al. 2018). However, empirical data on its validity in training contexts are scarce. This study evaluated compliance to three out of the four categories of the taxonomy (i.e., intrinsic-static, intrinsic-dynamic, and extrinsic-static) in a computerized training of VSA using different tasks (e.g., object completion, Tangram). We investigated the same six tasks, which Jung et al. (2020) reported fitting the taxonomy in testing situations employing the same digital MaGrid ${ }^{\circledR}$ application over ten weeks in 77 children (aged 50-63 months). In particular, we evaluated whether the same tasks used for training VSA, (1) fit the three categories and (2) predicted children's performance in a paper-pencil-based posttest. Contrary to previous findings, the pattern of children's training performance did not match the three categories of VSA as shown by confirmatory-factor analysis. This suggests that compliance of tasks to the taxonomy of VSA when used in testing situations not necessarily allows for generalization to training contexts. In the additional regression analyses, only performance for extrinsic-static VSA and the pre-test significantly predicted post-test performance. These results indicate that the translation of assessment tasks into training content requires additional theoretical and practical considerations.

\section{POSTERS: Submission 141}

Do People See Themselves as Fatter When an Artificial Intelligence Says so?

Thomas Chazelle ${ }^{1}$, Michel Guerraz ${ }^{1,2}$, Nicolas Morgado ${ }^{3}$, Richard Palluel-Germain ${ }^{1}$

${ }^{1}$ Laboratory of Psychology and NeuroCognition (LPNC), University of Grenoble Alpes, CNRS, Grenoble, France

${ }^{2}$ University of Savoie Mont Blanc, Chambéry, France

${ }^{3}$ Laboratoire sur les Interactions Cognition-Action-Émotion (LICAÉ), Université Paris Nanterre, France

Background: The idea that distortions in body perception can emerge from social influence is widespread, but lacks experimental support. Aims: We propose to test whether social information about weight can influence body representation with a new paradigm we called "guesser artificial intelligence" (AI). Methods: In a preregistered 
experiment, 599 participants (all females to avoid gender-related differences) were exposed to what they were told was an AI. This alleged AI, actually a basic algorithm, made either correct or random guesses about them. The program then randomly told them that they were either fat or thin. After this phase, participants selected the figure that best resembled them on a computer-generated scale and answered a body satisfaction questionnaire. We expected an interaction effect between accuracy of former guesses and weight information, with a stronger effect of information when the AI was thought to be reliable (i.e., made correct guesses) rather than unreliable (i.e., made random guesses). Results: We found neither the expected interaction effect nor any main effect. A manipulation check provided partial support in favor of our paradigm, as reliability groups strongly contrasted, but participants evaluated the AI that made accurate guesses as moderately reliable. Exploratory analyses revealed a moderate link $\left(\eta_{\mathrm{p}}{ }^{2}=0.078\right)$ between body overestimation and body dissatisfaction, that held beyond the effect of BMI. Conclusion: This link between overestimation and dissatisfaction contradicts recent claims that perceptual and attitudinal body image are fully independent. We propose ways to improve the guesser-AI paradigm.

\section{POSTERS: Submission 157}

\section{Meaning of a shape as a result of previous knowledge and axial rotation}

Jurgís Šķilters, Liğa Zariņa

Laboratory for Perceptual and Cognitive Systems, University of Latvia, Riga, Latvia

The same sensory data can generate different perceptions once changed in rotation. Although the top-down impacts on perception have been confirmed (Lupyan et al. 2020) it is less clear to what extent they matter in perceiving specific figural qualities. In our studies, we are examining the well-known diamond/square phenomenon (Palmer 1985). The aim of this research is to determine the impact of previous verbal and visual knowledge (combined with the angle of rotation) on the perception of particular shape. In betweengroups experiments (a) gaze movements have been recorded, (b) a selectional task conducted where square or diamond (in random set of stimuli rotated 15 degrees each) has to be selected. Both experiments are conducted in conditions with and without instructions (referring to square and diamond). After the task (b) visual and verbal knowledge of geometry has been tested. According to our results participants with a more detailed knowledge (both visual and verbal) show differences in categorizing the shape depending on its rotation degree. The case without instructions shows the impact of previous knowledge on the interpretation of all rotations (except the 0 degree condition). When examining the previous geometric knowledge, instructions significantly constrain differences in categorizing 30 and 45 degree rotation. The impacts of verbal instructions are also supported by the eye tracking experiments showing that instructions constrain a more focused distribution of fixations. Our findings allow to conclude that top-down impacts are significant but are co-determined by the rotational angle, previous knowledge, and the type of instruction.

\section{POSTERS: Submission 158}

\section{The relationship between allocentric neglect and extinction in stroke patients}

\section{Marika Mauti}

\section{Sapienza University of Rome, Italy}

Right cerebral hemisphere damages may result in extinction and/or spatial-egocentric/allocentric-neglect. Patients with extinction detect a stimulus if presented on one side, but not those presented on the left in a double simultaneous stimulation (Baylis et al. 1993). Egocentric and allocentric neglect impair the perception of the space on the left of the body midline and the left half of the object of focus, respectively (Vuilleumier et al. 1999). In the pre-attentive hypothesis, neglect weakens the ability to perceive an object by observing some of its features; in the attentive hypothesis, neglect affects the attentional modulation more broadly, with patients performing poorly in tasks that require high focal attention (Treisman \& Gelade 1980). Studies show that neglect and extinction are independent disorders (Vossel et al. 2011). However, most patients with neglect also show extinction. We run paper-and-pencil measurements and build ad hoc tests. We examine the relationship between the allocentric component of neglect and extinction. We also evaluate the pre-attentive component of commission and omission errors in the allocentric condition. Our sample consists of both mixed and pure allocentric or egocentric neglect patients. We manage to test patients with ego- and allo-centric neglect separately and find that structurally incomplete stimuli are marked as target (commission errors driven by the closer effect), consistently with the pre-attentive hypothesis. However, when stimuli presented pop-out details, commission errors are observed. Patients with allocentric neglect also show extinction, suggesting that the joint presence of egocentric neglect and extinction can explain allocentric neglect.

\section{POSTERS: Submission 169}

\section{Probing the spatial bias in hemispatial neglect using} a landmark task and fMRI

Ilenia Salsano ${ }^{1,2}$, Valerio Santangelo ${ }^{2,3}$, Alessandro Matano ${ }^{4}$, Rita Vadalà ${ }^{5}$, Chiara Briani ${ }^{2}$, Giovanni Giulietti ${ }^{2}$, Maria De Luca ${ }^{6}$, Carlo Caltagirone ${ }^{7,8}$, Gabriella Antonucci ${ }^{9}$, Barbara Spano ${ }^{2,10}$, Davide Nardo ${ }^{11}$

${ }^{1}$ Sapienza University of Rome, Italy

${ }^{2}$ Neuroimaging Laboratory, Santa Lucia Foundation IRCCS, Rome, Italy

${ }^{3}$ Department of Philosophy, Social Sciences \& Education, University of Perugia, Perugia, Italy

${ }^{4}$ Neuropsychology Center, IRCCS Santa Lucia Foundation, Rome, Italy

${ }^{5}$ NeuroRadiology, Santa Lucia Foundation IRCCS, Rome, Italy

${ }^{6}$ Neuropsychology Laboratory, IRCCS Santa Lucia Foundation, Rome, Italy

${ }^{7}$ Laboratory of Clinical and Behavioural Neurology, IRCCS Santa Lucia Foundation, Rome, Italy

${ }^{8}$ University "Tor Vergata" Rome, Rome, Italy

${ }^{9}$ Department of Psychology, Sapienza University of Rome, Italy 
${ }^{10}$ Technology and Training Methods for Disability Care Laboratory, Santa Lucia Foundation IRCCS, Rome, Italy ${ }^{11}$ MRC Cognition and Brain Sciences Unit, University of Cambridge, Cambridge, United Kingdom

Background: The landmark task is a neuroimaging version of the line bisection task, commonly used to diagnose hemispatial neglect in stroke patients. Neglect patients typically bisect a line showing a rightward deviation, due to the presence of a spatial bias. Aims: Here, we used the landmark task in fMRI to characterize the neural correlates of spatial bias in 22 patients with $(\mathrm{N}+; 9)$ or without neglect $(\mathrm{N}-; 13)$, and in 25 healthy controls (HC). Methods: Participants were presented with lines covering a visual angle of $18 \mathrm{deg}$., pre-bisected at either 0 , $0.5,1$ or $2 \mathrm{deg}$. (leftwards or rightwards), and were asked to indicate where the line was bisected (left, centre, right). Results: Consistent with previous findings, behavioural results showed a deficit in line bisection (rightward deviation) selectively in $\mathrm{N}+$. When imaging data were analyzed irrespective of side, $\mathrm{N}+$ showed that activity in attentionrelated regions increased with increasing distance from the midline; whereas $\mathrm{HC}$ and $\mathrm{N}-$ showed an inverse relationship. When data were analyzed by taking side into account, no group showed significant correlations along the rightward gradient (progressing from left to right). Conversely, along the leftward gradient, $\mathrm{N}+$ showed increased activity in attention-related and prefrontal areas, $\mathrm{N}$ - partial activity in attention-related areas, and $\mathrm{HC}$ no activity at all. Conclusion: Our results suggest that, besides the spatial bias, a rescaling process of spatial representations may occur in $\mathrm{N}+$. Moreover, neurocognitive resources in $\mathrm{N}+$ seem to be allocated as a function of stimulus positioning along a continuum, across the ipsilesional/contralesional sides.

\section{POSTERS: Submission 171}

\section{Applying a Healing Process to Hospital Wayfinding Systems during COVID-19}

\section{Yasmin Garcia-Sterling \\ University College London, National Health Service (NHS) England, Capital Hospitals London (CHL) projects part of HCP, United Kingdom}

Background: Wayfinding, the process of positioning, planning and following a route is vital to remain consciously efficient in Covid-19, including factoring. Way findings cognitive biases under-researched. Using novel mixed-methods the research explores what information hospital end-users have used when moving through their local hospitals to reach their destination during the pandemic. Aims: This research questioned how human thinking has played a prominent part in facilitating our choice of direction. In line with NHS Englands aim to protect the most vulnerable mental health patients during a pandemic. Facilities strategise ways to mitigate negative Wayfinding experiences and promote health and healing environmental movement within hospitals, supporting end-user behaviour. Methods: Local community charity's are used to collect 400 questionnaires, followed from weekly webinars on spatial awareness bringing an association of trust via community engagement. Using social and semantic analysis tools and hospital spatial-temporal pandemic mobility data to mitigate Covid-19 spread as a main issue to governments. Results: The results available July-August, 2021, will poster presents the correlation of end-user awareness to current Wayfinding in local hospitals. Among them, will display the types of risks that are important to understand and what cognitive biases have availed in hospital settings during these uncertain times. Conclusion: In part of a wider PhD and NHS sponsored research to digitally integrate a Wayfinding system in St.Barts hospitals, shifts Wayfinding into a user-aware system. Understanding benefits from a humanistic perspective, this posters collected data will follow into action research to digitally design Wayfinding Systems embedding systematic errors and wellbeing influence.

\section{POSTERS: Submission 178}

\section{Horizontal and vertical spatial associations of serial order in working memory}

\section{Matthias Hartmann, Corinna S. Martarelli, Nils R. Sommer}

\section{UniDistance Suisse, Switzerland}

Background: Maintaining serial order in working memory is crucial for cognition. Recent theories propose that serial information is achieved by positional coding of items on a spatial frame of reference. In line with this, an early-left and late-right spatial-positional association of response code (SPoARC) effect has been established, although the exact format of the spatialization of serial order remains unclear. Aim and Methods: In this study, we investigated SPoARC effects along the horizontal and vertical spatial dimension. The task followed a three-step procedure. In Step 1, participants memorized a four-letter sequence consisting of two consonants and two vowels (e.g., curi). In the critical Step 2, single letters were presented in the center of the screen and participants performed a brief consonantvowel classification task by means of saccadic responses (either leftward-rightward or upward-downward). In Step 3, participants were required to reproduce the sequence in the correct order. This three-step procedure was repeated 36 times. Mean saccade latencies for each serial position of the letter in the memorized sequence (1-4) per response direction were analyzed by means of a Bayesian repeated measure linear regression analysis. Results and Conclusion: We replicated the left-to-right horizontal SPoARC effect and established an up-to-down vertical SPoARC effect. The direction of the vertical SPoARC effect (early-up) was in contrast to that predicted by metaphor theory, polarity correspondence, or by the indirect spatialnumerical association effect (late-up). Rather, our results support the mental whiteboard-hypothesis, according to which positions can be flexibly coded on an internal space depending on the task demands.

\section{POSTERS: Submission 182}

\section{Improved ability of object detection using echolocation by a repetitive task}

\section{Tomoki Maezawa, Jun Kawahara}

\section{Hokkaido University, Japan}

The ability to localize spatial and non-spatial features of surrounding objects from echoic cues has been acknowledged as echolocation. A number of studies found that sighted human without visual impairment can improve skills to echolocate objects after experiencing short-period (e.g., second days) of audio-spatial tasks. However, the amount of training to improve echolocation ability has been unknown, resulting in the lack of systematic protocols to establish echolocation training. The present study examined whether echolocation ability achieved during a repetitive task over 2 days improves and persists for at least one month. Sighted participants identified target presence (or absence) using $10 \mathrm{~s}$ of $4 \mathrm{kHz}$ clicks produced through a loudspeaker. The target object was placed from 20 to $50 \mathrm{~cm}$ in front of the participants. The object detection task lasted 120 trials (approximately 50 min) per block. The tasks were divided into three blocks. Specifically, the participants completed the second block 1 to 8 days after performing the first block. The third block was administered 30-137 days after performing the second block. The results indicated that 
participants improved their detection ability following two days of repetitive tasks. Importantly, the improvement achieved on the second block was maintained for at least 30 days without any additional trainings. The participants altered their decision criterion depending on the task duration and target distances, and the criterion shifting would contribute to the enhancement of echolocation performance. We suggest that sighted participants can persist echolocation ability for one month after the two-days training.

\section{POSTERS: Submission 186}

\section{Pseudoneglect in children and young adults evaluated by means of the E-BTT}

\author{
Federica Somma ${ }^{1}$, Antonietta Argiuolo ${ }^{1}$, Michela \\ Ponticorvo $^{1}$, Orazio Miglino ${ }^{1}$, Paolo Bartolomeo ${ }^{2}$, \\ Onofrio Gigliottal
}

${ }^{1}$ Natural and Artificial Cognition Laboratory, Department of Humanities, University of Naples "Federico II", Naples, Italy

${ }^{2}$ Sorbonne Université, Inserm U 1127, CNRS UMR 7225, Paris Brain Institute, ICM, Hôpital de la Pitié Salpêtrière, 75013 Paris, France

Background: E-TAN platform, connecting tangible environments of visuospatial tasks to digital data collection, is used to enhance the Baking Tray Task (E-BTT), an ecological visuospatial task that requires to uniformly place 16 objects on a rectangular tray. The E-BTT, being able to digitally trace performances on the baking tray task, allows to obtain a rich set of data. Aims: The aim of the present study was to administer the E-BTT task for the first time to school-age children and compare their performances to a sample of young adults who showed pseudoneglect, a well-known leftward preference. Methods: 51 school-aged children and 94 university students participated in the study. A cognitive assessment and the E-BTT task were administered. The space was virtually divided into four quadrants and the positions of the first placed object were analysed. Results: The results show a statistically significant preference of the children to start placing objects in the lower right portion. A statistically significant difference emerges between young adults and children on the first positioning with respect to the four quadrants $(p<.0001)$; moreover children frequently start from the right quadrants, on the contrary, young adults tend to place the first object in the left quadrants $(p<.0001)$. Conclusion: Our preliminary results show, for the first time, that performances on E-BTT (in terms of the positions of the first placed object/bun) support what has been reported in the literature on pseudoneglect regarding other tasks: that it gradually emerges during development in relation to biological, cultural and biomechanical factors.

\section{POSTERS: Submission 187}

\section{How do our demographics shape our trajectories?}

\section{Hippolyte Dubois, Patrick Le Callet, Antoine Coutrot}

\section{Université de Nantes, France}

How people navigate a new environment is influenced by their demographics (e.g. age or culture) or clinical profile (e.g. dementia). This has been notably investigated in the Sea Hero Quest (SHQ) project, a spatial navigation video game that collected trajectories from over 4 millions players worldwide. So far, SHQ data has only been analysed with classical yet limited statistical tools by interdisciplinary research teams. To further the analysis, we aim to identify outlying behaviour, given the socio-demographic characteristics of the corresponding player. For this first AI-powered analysis pipeline, we focus on easily interpretable machine learning techniques, and also provide a generic blackbox approach, used in conjunction with new ad hoc trajectory metrics. Each "player" is characterized by a set of sociodemographic characteristics, as well as several metrics computed from their spatial navigation trajectory. We use $5 \%$ of samples to identify outliers using Isolation Forest algorithm on the combination of metrics. We split the data in two training and testing sets, with a 4-to-1 ratio, with demographics as input, and outlier label as output. We train the classifiers on the training set and evaluate them on the testing set using Cohen Kappa Score. We demonstrate that the Random Forest algorithm predicts the outlierness of a sample with a $0.976 \pm 0.002$ Cohen Kappa, and measure the importance of each demographic feature. Comparatively to previous research, we were able to show the influence of more demographic features using novel ad-hoc metrics.

\section{POSTERS: Submission 188}

\section{A decrease of the tool effect in older adults}

Marion Luyat ${ }^{1}$, Kévin Dumez ${ }^{2}$, Myriam Noèl ${ }^{1}$, Emin Altintas ${ }^{1}$, Cédric Campion ${ }^{3}$, Michel Guerraz ${ }^{4}$

${ }^{1}$ Univ. Lille, laboratory, Lille, France

${ }^{2}$ Clinique du Val de Lys (Groupe Ramsay), Tourcoing, France

${ }^{3}$ Lens Hospital Center, 99 route de la Bassée, France

${ }^{4}$ Laboratory of Psychology and Neurocognition, CNRS UMR 5105, Univ. Savoie Mont Blanc (USMB), France

Background: Holding a phone or grasping an object are daily activities that require internal model of action, based on body segments parameters: the body schema. In young adults, the perceived limits of reachability are augmented when and even after using a tool (e.g. a rake). This is interpreted as the result of an extension of the body schema (embodiment), reflecting its high plasticity at least in young individuals. Aims: The goal of our research is to test the possible defect of body schema updating process in older adults by using the tool-effect task. Methods: Forty-six young participants, 20 non-demented older participants and 37 older participants with cognitive impairment took part in the experiment. The task consisted in visually judging the possibility to reach targets positioned at different locations on a table before and after using a rake. Results: By contrast to the younger group, we observed a great decrease in the tool effect in the older participants group and even more so for the participants with neurocognitive disorders, although no significant difference was found between these latter two groups. Conclusion: A decrease in the tool effect was found with advanced age, suggesting a reduced plasticity of the body schema and therefore of motor-action programs. This could enhance the risk of loss of postural control due to misjudgments of intended actions. Thus, the "tool-effect" task could serve both to detect those in high risk of fall and to evaluate the improvement of the plasticity of the motor-action model in rehabilitation process. 


\section{POSTERS: Submission 193}

\section{Aesthetic appreciation in art context. Human versus AI authorship}

Salvatore Gaetano Chiarella, ${ }^{1,2}$, Dionigi Mattia

Gagliardi $^{2,3}$, Giulia Torromino ${ }^{2}$, Dario Rossi ${ }^{4,5}$, Fabio Babiloni $^{5,6,7}$, Giulia Cartocci ${ }^{5,6}$

${ }^{1}$ Department of Psychology, Sapienza University of Rome, Italy

2Numero Cromatico, Rome, Italy

3Department of Graphic and communication design, Quasar Insititute for Advanced Design, Rome, Italy

4Department of Business and Management, LUISS Guido Carli

University, Rome, Italy

5 BrainSigns srl, Rome, Italy

6Department of Molecular Medicine, Sapienza University of Rome, Italy, Italy

7Department of Computer Science and Technology, Hangzhou Dianzi University, Xiasha Higher Education Zone, Hangzhou, China

Background: Creativity, intended as an exclusive human activity, has been questioned by the growing implementation of machine learning and artificial neural networks in the field of Artificial Intelligence (AI), recently used for art production. In addition, recent studies showed that human aesthetic appreciation can be modulated by several factors ranging from expectations to context. Aims: We investigated whether, in an ecological environment, prior knowledge about authorship modulates aesthetic appreciation of two paintings. Specifically, we compared judgement of pre-assigned Human- versus AI-authorship on two human-made abstract paintings between two groups $(\mathrm{N}=73)$ based on presentation order. Methods: The data collection took place within the context of the art fair "ArtVerona". Participants observed two consecutive abstract paintings being informed of the authorship just before watching it and expressed an aesthetic judgment on a five-point Likert scale. The order of painting's presentation and the pre-assigned authorship were counterbalanced. During the entire task the galvanic skin response (GSR) was recorded. Results: We observed a presentation order effect showing that when the pre-assigned Human-painting was shown as first, judgement on the subsequent pre-assigned AI-painting was lower; while the two paintings received equal aesthetic judgements when AI was seen first. In parallel, results of the GSR showed that participants were more activated during the second presentation, irrespective of pre-assignment. Conclusion: Our results highlight two findings: first that participants experienced the second presentation as a moment in which to 'compare' the two paintings; second that there is a negative bias for AI-made artworks compared to humans.

\section{POSTERS: Submission 195}

\section{Updating SOT with a Dynamic Oriented Integrated Rehabilitative (DORIS) system}

\section{Maurizio Petrarca ${ }^{l}$, Susanna Summa ${ }^{l}$, Martina Favetta $^{l}$, Gessica Vasco ${ }^{I}$, Simone Gazzellini ${ }^{1}$, Enrico Castelli ${ }^{1}$, Alain Berthoz ${ }^{2}$ \\ ${ }^{1}$ Bambino Gesù Children's Hospital-IRCCS, Rome, Italy ${ }^{2}$ College de France, Paris}

Background: Nashner L.M. described the Sensory Organization Test (SOT) in 1982, and the first commercial solution arrived in 1986. It is known that this condition of balance testing is more effective than using only force plates information. Nevertheless, a long time passes during which the technology grow, offering new opportunities. Aims: The main aim was to enhance the opportunity to study sensory-motor integration in normally developed children and children developed with different pathological conditions. An associated aim was to study sensory fusion development in children. Methods: A new integrated system was developed ad-hoc. DORIS is a versatile solution that can apply both for research purposes and personalise equilibrium and gait training. The DORIS system consists of a 6 DoF Stewart robotics platform, controlled simulating mass-spring dumper systems and in position, integrated in real-time with a customised immersive virtual system, a 3D motion capture system, surface EMG, robotics orthosis and IMUs. Results: Combining the real-time feature of the system is possible to realize both sudden and predictable postural solicitation. It becomes possible to observe anticipatory, predictable, and reactive strategies during gait and standing in different sensory and dynamic conditions strictly linked with peripersonal and extrapersonal space perception, being the platform camouflaged at floor level along the pathways of the Gait Laboratory. Conclusion: The adopted ad hoc solution allows the building of a structured battery test to assess different scientific questions on sensory-motor integration in children with pathological conditions.

\section{POSTERS: Submission 202}

\section{The effect of spatio-temporal sequential learning on covert selective attention}

\section{Marta Szewczyk, Pawet Augustynowicz, Piotr Francuz \\ The John Paul II Catholic University of Lublin, Poland}

Facilitation effects of sequential learning on overt attention are well known. However, a similar guiding effect in spatio-temporal dimensions for covert selective attention has not been thoroughly investigated. We designed an ERP experiment to check (1) whether implicitly learned predictable sequence of target locations and target onset time facilitates covert target localization; (2) which stage of processing is facilitated: perceptual, attentional, or response selection-as measured by the P1\&N1, N2pc and sLRP latencies, respectively. We analyzed data from 15 healthy adults. Their task was to keep the gaze fixated in the center of the visual field and to indicate whether a target surrounded by distractors appeared on the left or the right side. Unbeknown to the participants, the target followed a predictable spatial, temporal or spatio-temporal pattern. Reaction times and EPRs latencies were compared to the control block where stimuli appeared randomly in space and time. Reaction times were faster for spatially and spatio-temporally predictable targets. However, predictable sequence of target onset time did not allow for faster target localization. We also found shorter N2pc latency for targets that followed predictable sequence only in the spatial dimension. This was the first study to show the effect of implicitly learned predictability in spatial dimension on a faster attentional selection (N2pc), but not on the speed of motor processes. We discuss the results in the predictive coding theory framework.

\section{POSTERS: Submission 209}

\section{Event segmentation through the lens of multimodal interaction}

Vipul Nair ${ }^{1}$, Jakob Suchan ${ }^{2}$, Mehul Bhatt ${ }^{3}$, Paul Hemeren ${ }^{1}$

${ }^{1}$ School of Informatics, University of Skövde, Sweden

${ }^{2}$ University of Bremen, Germany 
${ }^{3}$ School of Science and Technology, Örebro University, Sweden CoDesign Lab, Sweden

Research in naturalistic event perception highlights the significance of visuospatial attributes pertaining to everyday embodied human interaction. This research focuses on developing a conceptual cognitive model to characterise the role of multimodality in human interaction, its influence on visuospatial representation, event segmentation, and high-level event prediction. Our research aimed to characterise the influence of modalities such as visual attention, speech, hand-action, body-pose, head-movement, spatial-position, motion, and gaze on judging event segments. Our particular focus is on visuoauditory narrative media. We select 25 movie scenes from a larger project concerning cognitive film/media studies and performing detailed multimodal analysis in the backdrop of an elaborate (formally specified) event analysis ontology. Corresponding to the semantic event analysis of each scene, we also perform high-level visual attention analysis (eye-tracking based) with 32 participants per scene. Correlating the features of each scene with visual attention constitutes the key method that we utilise in our analysis. We hypothesise that the attentional performance on event segments reflects the influence exerted by multimodal cues on event segmentation and prediction, thereby enabling us to explicate the representational basis of events. The first results show trends of multiple viewing behaviours such as attentional synchrony, gaze pursuit and attentional saliency towards human faces. Work is presently in progress, further investigating the role of visuospatial/ auditory cues in high-level event perception, e.g., involving anticipatory gaze vis-a-vis event prediction. Applications and impact of this conceptual cognitive model and its behavioural outcomes are aplenty in domains such as (digital) narrative media design and social robotics. 


\section{Author Index}

For each author reference is made to the type of contribution (KEYNOTE, SYMPOSIUM, TALKS, POSTERS) and to the submission number.

\begin{tabular}{|c|c|}
\hline Abusham, M. & TALKS: 145 \\
\hline Adhikari, A. & TALKS: $100,151,174$ \\
\hline Agostini, F. & SYMPOSIUM 10: 140 \\
\hline Aguilar, I. & TALKS: 100 \\
\hline Ahumada, F. & SYMPOSIUM 10: 130 \\
\hline Aktaş Yanaş, E. & TALKS: 92 \\
\hline Albrecht, R. & SYMPOSIUM 14: 42 \\
\hline Altintas, E. & POSTERS: 188 \\
\hline Altomare, E. C. & SYMPOSIUM 3: 205 \\
\hline Andreatta, M. & SYMPOSIUM 5: 99 \\
\hline Angioletti, L. & POSTERS: 4 \\
\hline Ansah, G. & TALKS: 139 \\
\hline Antonucci, G. & $\begin{array}{l}\text { SYMPOSIUM 3: General abstract, 198; } \\
\text { POSTERS: } 169\end{array}$ \\
\hline Arendtsen, B. & TALKS: 126 \\
\hline Argiuolo, A. & POSTERS: 34, 186 \\
\hline $\begin{array}{l}\text { Augustynowicz, } \\
\text { P. }\end{array}$ & POSTERS: 202 \\
\hline Avanzini, $\mathrm{P}$. & SYMPOSIUM 6: 18 \\
\hline Avesani, $\mathrm{P}$. & TALKS: 87 \\
\hline Aydin, M. & SYMPOSIUM 3: 198, 203 \\
\hline Babiloni, F. & POSTERS: 193 \\
\hline Baker, S. & TALKS: 126 \\
\hline Balconi, M. & TALKS: 7; POSTERS: 4, 11, 55 \\
\hline Baldauf, D. & TALKS: 87 \\
\hline Bao, Y. & $\begin{array}{l}\text { SYMPOSIUM 8: General abstract, 119; TALKS: } \\
\quad 113,114,153\end{array}$ \\
\hline Bardi, L. & SYMPOSIUM 4: 124 \\
\hline Bartolomeo, P. & SYMPOSIUM 9: 201; POSTERS: 34, 186 \\
\hline Battaglia, S. & SYMPOSIUM 12: 57 \\
\hline Bedini, M. & TALKS: 87 \\
\hline Berthoz, A. & POSTERS: 195 \\
\hline Berti, A. & KEYNOTE 9 \\
\hline Besada, J. L. & SYMPOSIUM 5: General abstract, 48, 60, 99 \\
\hline Bhatt, M. & TALKS: 162; POSTERS: 209 \\
\hline Bianco, F. & SYMPOSIUM 9: 199 \\
\hline Bisesi, E. & SYMPOSIUM 5: 91, 93, 99 \\
\hline Bisiacchi, P. S. & TALKS: 53 \\
\hline Blanke, O. & KEYNOTE 7 \\
\hline Blini, E. & TALKS: 177 \\
\hline $\begin{array}{l}\text { Blom Andersen, } \\
\text { N. }\end{array}$ & TALKS: 126 \\
\hline Bodenheimer, B. & TALKS: 69,96 \\
\hline $\mathrm{B} ø \mathrm{hm}, \mathrm{M}$. & TALKS: 126 \\
\hline Bolis, D. & SYMPOSIUM 4: 159 \\
\hline Bollini, A. & SYMPOSIUM 13: General abstract; TALKS: 29 \\
\hline Bonato, M. & TALKS: 177 \\
\hline Bonini, L. & SYMPOSIUM 11: 181 \\
\hline
\end{tabular}

\begin{tabular}{|c|c|}
\hline Bonvento, M. & POSTERS: 22 \\
\hline Borghi, A. & TALKS: 128 \\
\hline Bosco, A. & TALKS: 106 \\
\hline Bottini, R. & SYMPOSIUM 7: 82; POSTERS: 63 \\
\hline Bräuning, D. & POSTERS: 133 \\
\hline Bremner, A. & SYMPOSIUM 13: 120 \\
\hline Bretin, A. & TALKS: 151 \\
\hline Briani, C. & POSTERS: 169 \\
\hline Brookes, D. & TALKS: 126 \\
\hline Brouillet, D. & POSTERS: 67 \\
\hline Brozzoli, C. & $\begin{array}{l}\text { SYMPOSIUM 11: General abstract, 156, 185; } \\
\text { SYMPOSIUM 12: } 150\end{array}$ \\
\hline Bruni, L. E. & TALKS: 197 \\
\hline Bucciarelli, M. & TALKS: 15 \\
\hline Buck, L. & TALKS: 69 \\
\hline Bunel, M. & SYMPOSIUM 1: 90 \\
\hline Burleigh, A. & TALKS: 154 \\
\hline Caffò, A. O. & TALKS: 106 \\
\hline Cai, M. & SYMPOSIUM 8: 167 \\
\hline Calafiore, $\mathrm{C}$. & TALKS: 200 \\
\hline Caltagirone, $\mathrm{C}$. & POSTERS: 169 \\
\hline Camarda, D. & TALKS: 191 \\
\hline Campion, $\mathrm{C}$. & POSTERS: 188 \\
\hline Campus, $\mathrm{C}$. & TALKS: 173; POSTERS: 64 \\
\hline $\begin{array}{l}\text { Canales-Johnson, } \\
\text { A. }\end{array}$ & SYMPOSIUM 10: 148 \\
\hline Candelise, C. & SYMPOSIUM 3: 198 \\
\hline Candini, M. & SYMPOSIUM 12: 57 \\
\hline Cantarella, G. & TALKS: 53 \\
\hline Cappagli, G. & SYMPOSIUM 13: 31 \\
\hline Carravieri, E. & TALKS: 33 \\
\hline Carré, O. & TALKS: 110, POSTERS: 108 \\
\hline Cartaud, A. & SYMPOSIUM 12: 180 \\
\hline Cartocci, G. & POSTERS: 193 \\
\hline Caruana, F. & SYMPOSIUM 6: 18 \\
\hline $\begin{array}{l}\text { Casado Palacios, } \\
\text { M. }\end{array}$ & POSTERS: 64 \\
\hline Casagrande, $\mathrm{M}$. & SYMPOSIUM 10: 140 \\
\hline Cassioli, F. & TALKS: 7; POSTERS: 11,55 \\
\hline Castelli, E. & POSTERS: 195 \\
\hline Catherwood, D. & TALKS: 126 \\
\hline Cerqueglini, L. & POSTERS: 97 \\
\hline Cesari, P. & TALKS: 116 \\
\hline Chakraborty, S. & TALKS: 96 \\
\hline Chamberlain, B. & SYMPOSIUM 2: 176; TALKS: 145 \\
\hline Chao, I. & SYMPOSIUM 3: 203 \\
\hline Chatterjee, A. & KEYNOTE 8 \\
\hline Chazelle, T. & POSTERS: 141 \\
\hline Chemero, A. & KEYNOTE 4 \\
\hline
\end{tabular}


Chen, S.

SYMPOSIUM 8: 137

Chen, $\mathrm{X}$

TALKS: 117

Chiarella, S. G.

SYMPOSIUM 9: 189, 192; POSTERS: 193

Chrenek, E. SYMPOSIUM 3: 203

Chrispin, A.

TALKS: 107

Chumaira, T. M. TALKS: 197

Cioffi, F. POSTERS: 79

Cocchi, E. $\quad$ SYMPOSIUM 13: 49

Coello, Y. SYMPOSIUM 12: 180

Colautti, L. TALKS: 33

Colombari, E. TALKS: 116

Committeri, G. SYMPOSIUM 3: 205; TALKS: 204

Contemori, G. TALKS: 177

Contreras, M. J. POSTERS: 9

Cornu, V. POSTERS: 133

Corrias, G. TALKS: 211

Coutrot, A. POSTERS: 187

Creem-Regehr, S. SYMPOSIUM 2: 176; TALKS: 96, 145

Cristoforetti, G. SYMPOSIUM 7: 39

Crivelli, D. TALKS: 7

Curatti, S. TALKS: 211

Cutini, S. TALKS: 172

Cuturi, L. F. TALKS: 76

Dagba, J. TALKS: 51

Dai, S. TALKS: 107, 110; POSTERS: 108

d'Avella, A. SYMPOSIUM 3: 207

Davoine, P. TALKS: 107

De Giorgis, V. TALKS: 173

De Luca, M. POSTERS: 169

De Pasquale, P. $\quad$ SYMPOSIUM 3: 207

Delogu, F. TALKS: 211

Díaz-Kommonen, TALKS: 197 L.

Di Matteo, R. TALKS: 204

di Pellegrino, G. SYMPOSIUM 12: 57

Diaine, F. TALKS: 110; POSTERS: 108

Djebbara, Z. $\quad$ SYMPOSIUM 6: 6

Dubois, H. POSTERS: 187

Dumez, K. POSTERS: 188

Dupierrix, E. TALKS: 107

Dupin, L. TALKS: 45

Dureux, A. SYMPOSIUM 12: 71

Duroisin, N. TALKS: 155,161

Durupt, C. TALKS: 51

Dutriaux, L. POSTERS: 63

Edgar, G. TALKS: 126

Ehrsson, H. H. SYMPOSIUM 12: 150

Emo, B. TALKS: 170

Ernst, M. SYMPOSIUM 13: 49

Esmail, S. $\quad$ SYMPOSIUM 3: 203

Esposito, D. TALKS: 29

Fan, Y. POSTERS: 129, 131
Fanari, R.

Farnè, A

Farquet, G.

Farroni, T.

Fasce, M.

Favetta, M.

Favieri, F.

Feraco, T.

Fernández-

Méndez, L. M.

Fernberg, P.

Ferrara, A.

Ferri, R.

Fias, W.

Figueras Masip, A.

Fikke, R. C.

Fini, C.

Fink, P.

Firouzeh, P.

Fischer, M. H.

Forte, G.

Fortmann, O.

Foulsham, T.

Fragueiro, A.

Fraisse, $\mathrm{P}$.

Frampton, Y.

Francuz, P.

Frassinetti, F.

Frisoni, M.

Fronda, G.

Fusaro, M.

Fuste Castella, R. TALKS: 126

Gagliardi, D. M. POSTERS: 193

Gail, A.

SYMPOSIUM 11: 175

Gallo, F.

TALKS: 10

Gamberini, L.

Ganczarek, J.

TALKS: 5

Garcia-Sterling,Y. POSTERS: 171

Garrett, B.

TALKS: 145

Gautheron, F.

Gaveau, V.

Gazzellini, S.

Geertsema, T.

Gigliotta, O.

Giordano, V.

Giovannoli, J.

Giuberti, V.

Giudice, N.

TALKS: 211

TALKS: 170

SYMOSIUM 13: 46

POSTERS: 195

SYMPOSIUM 10: 140

POSTERS: 22

SYMPOSIUM 10: 140

TALKS: 126

SYMPOSIUM 3: 203

TALKS: 58

TALKS: 200

POSTERS: 67

SYMPOSIUM 12: 57

S: 204

TALKS: 61

TALKS: 210

POSTERS: 195

TALKS: 126 POSTERS: 34, 186

SYMPOSIUM 10: 140

SYMPOSIUM 10: 140

SYMPOSIUM 12: 57

TALKS: 146
11: General abstract, 156, 185; SYMPOSIUM 12: General abstract; TALKS:

SYMPOSIUM 2: 176; TALKS: 145

SYMPOSIUM 7: General abstract, 39

SYMPOSIUM 4: General abstract; TALKS: 128

SYMPOSIUM 9: General abstract, 201; 


\begin{tabular}{|c|c|c|c|}
\hline Giulietti, G. & POSTERS: 169 & Kunz, A. & SYMPOSIUM 2: 101 \\
\hline \multirow[t]{2}{*}{ Gori, M. } & \multirow{2}{*}{$\begin{array}{l}\text { SYMPOSIUM 13: General abstract, 31, 49, 54; } \\
\text { TALKS: 29, 76, 173; POSTERS: } 64\end{array}$} & Kütt, T. & TALKS: 126 \\
\hline & & Kwarteng, E. A. & TALKS: 139 \\
\hline Grassi, M. & TALKS: 7 & Labeye, E. & TALKS: 184 \\
\hline Grison, E. & TALKS: 51 & Lacquaniti, F. & SYMPOSIUM 3: 207 \\
\hline Guegan, J. & TALKS: 132 & Lafitte, R. & TALKS: 107, 110; POSTERS: 108 \\
\hline Guelton, B. & SYMPOSIUM 1: General abstract, 75 & Lázár, I. & POSTERS: 56 \\
\hline Guerraz, M. & POSTERS: 141,188 & Le Callet, P. & POSTERS: 187 \\
\hline Guichaoua, C. & SYMPOSIUM 5: 99 & Legrain, $\mathrm{P}$. & SYMPOSIUM 5: 93 \\
\hline Gül, L. F. & TALKS: 92 & Letailleur, A. & SYMPOSIUM 5: 93 \\
\hline Gulhan, D. & POSTERS: 74 & Lhuillier, S. & TALKS: 127 \\
\hline Györkö, E. & POSTERS: 36 & $\mathrm{Li}, \mathrm{Y}$. & TALKS: 144 \\
\hline Gyselinck, V. & TALKS: 127,132 & $\mathrm{Li}, \mathrm{X}$. & POSTERS: 131 \\
\hline Hadizadeh, M. & SYMPOSIUM 3: 203 & Lin, $\mathrm{X}$. & SYMPOSIUM 8: 102 \\
\hline Hadj-Bouziane, F. & SYMPOSIUM 12: General abstract, 71 & Lin, $\mathrm{C}$. & SYMPOSIUM 8: 167 \\
\hline Hamburger, K. & SYMPOSIUM 14: 52 & Lisi, M. P. & SYMPOSIUM 4: 147 \\
\hline Harding, R. & TALKS: 196 & Liu, Y. & SYMPOSIUM 8: 119; TALKS: 153 \\
\hline Hartmann, M. & POSTERS: 178 & Lopez, A. & TALKS: 106 \\
\hline Hashemian, A. M. & TALKS: 100, 174 & Lucero, B. & SYMPOSIUM 10: General abstract, 130, 148 \\
\hline Haun, A. & TALKS: 14 & Luo, Y. & TALKS: 114,117 \\
\hline Hazebroek, J. & TALKS: 126 & Luyat, M. & POSTERS: 188 \\
\hline $\mathrm{He}, \mathrm{C}$. & SYMPOSIUM 5: 101 & Maeda, N. & SYMPOSIUM 3: 203 \\
\hline Hegarty, M. & SYMPOSIUM 5: 101 & Maezawa, T. & POSTERS: 182 \\
\hline Hemeren, $\mathrm{P}$. & POSTERS: 209 & Maffei, L. & POSTERS: 77,79 \\
\hline Hoelscher, C. & TALKS: 170 & Magenes, S. & TALKS: 33 \\
\hline Hofmann, P. & POSTERS: 8 & Malyshevskaya, & TALKS: 10 \\
\hline Holm, L.O.C.N. & TALKS: 126 & A. & \\
\hline Huang, Y. & TALKS: 123 & Mammarella, I. & POSTERS: 9 \\
\hline Huet, N. & TALKS: 184 & Marchegiani, L. & SYMPOSIUM 9: 199 \\
\hline Iachini, $\mathrm{T}$. & SYMPOSIUM 12: General abstract, 65; TALKS: & Marocco, D. & POSTERS: 34 \\
\hline & 62, 47; POSTE & Marotta, A. & SYMPOSIUM 10: 140 \\
\hline Ianì, $\mathrm{F}$. & TALKS: 15 & Marre, Q. & TALKS: 184 \\
\hline Ishikawa, $\mathrm{T}$. & KEYNOTE 3 & Martarelli, C. S. & POSTERS: 178 \\
\hline Iosa, $\mathrm{M}$. & SYMPOSIUM 3: General abstract, 198 & Martella, D. & SYMPOSIUM 10: 140 \\
\hline Izadifar, M. & POSTERS: 30 & Martínez-Molina, & POSTERS: 9 \\
\hline Jansen, P. & POSTERS: 8,13 & A. & \\
\hline Jeager, M. & TALKS: 107 & Martolini, C. & SYMPOSIUM 13: 31, 49 \\
\hline Jeffery, K. & KEYNOTE 5 & Maselli, A. & SYMPOSIUM 3: 207 \\
\hline Josa, R. & TALKS: 128 & Mastrodonato, G. & TALKS: 191 \\
\hline Jost, L. & POSTERS: 13 & Masullo, M. & POSTERS: 77, 79 \\
\hline Jung, S. & POSTERS: 133 & Matano, A. & POSTERS: 169 \\
\hline Kaijima, M. & TALKS: 170 & Mauti, M. & POSTERS: 158 \\
\hline Kan-Kilic, D. & TALKS: 59 & Meinhardt, A. & POSTERS: 133 \\
\hline Kasek, R. & POSTERS: 56 & Mellini, G. & SYMPOSIUM 12: 57 \\
\hline Kawahara, J. & POSTERS: 182 & Melloni, L. & TALKS: 14 \\
\hline Király, G. & POSTERS: 36 & Meneghetti, C. & SYMPOSIUM 2: General abstract, 32; TALKS: \\
\hline Kocabiyik, S. & TALKS: 59 & & 44; POSTERS: 9, 22 \\
\hline Kondyli, V. & TALKS: 162 & Mengue-Topio, $\mathrm{H}$. & TALKS: 155,161 \\
\hline Kónya, A. & POSTERS: 56 & Michalland, A. & SYMPOSIUM 4: 66; TALKS: 128; POSTERS: \\
\hline Kostov, K. & TALKS: 152 & Miglino, O. & SYMPOSIUM 9: 201; POSTERS: 34, 186 \\
\hline Kruijff, E. & TALKS: $100,151,174$ & $\begin{array}{l}\text { Milginno, U. } \\
\text { Miklashevsky, A. }\end{array}$ & $\begin{array}{l}\text { SYMPOSIUM 9: 201; POSTERS: 34, } 186 \\
\text { TALKS: } 58\end{array}$ \\
\hline Krukar, J. & SYMPOSIUM 14: 98 & & \\
\hline
\end{tabular}


Minear, M.

TALKS: 70

Miola, L. SYMPOSIUM 2: 32

Moeller, K. POSTERS: 133

Möhring, W.

TALKS: 68

Montani, V.

TALKS: 116

Morelli, F.

TALKS: 173

Morgado, N.

POSTERS: 141

Morgagni, S.

TALKS: 51

Morone, G.

SYMPOSIUM 3: 198

$\mathrm{Mu}, \mathrm{N}$.

TALKS: 153

Muffato, V. SYMPOSIUM 2: 32; TALKS: 44

Muñoz-Quezada, M.

Myachykov, A.

Nair, V.

TALKS: 10

Nardini, M.

POSTERS:209

Nardo, D.

KEYNOTE 1

Naughtie, C.

POSTERS: 169

Navarro, B.

TALKS: 126

Nenna, F.

POSTERS: 67

Nguyen-Vo, T.

TALKS: 5

Nitsche, M.

TALKS: 174

TALKS: 160

Noël, M.

POSTERS: 188

Nori, R. POSTERS: 41

Nuhn, E. SYMPOSIUM 14: General abstract, 43

Ognibene, D. SYMPOSIUM 9: 199; TALKS: 200

Olivetti, E.

TALKS: 87

Orioli, G.

SYMPOSIUM 13: 120

Orti, R.

TALKS: 47

Oztok, G.

POSTERS: 74

Pagán Cánovas, C. SYMPOSIUM 5: 60

Palluel-Germain, POSTERS: 141 R.

Palmer, B.

POSTERS: 38

Palmieri, A.

POSTERS: 77

Paolucci, S.

Papale, P.

SYMPOSIUM 3: 198

Parisi, I.

SYMPOSIUM 6: 17

Pasala, S. K.

SYMPOSIUM 13: 120

Pascucci, F.

Patanè, I.

Pavani, F.

Pazouki, T.

Saracini, C.

Sartorato, N.

Satyavarapu, G.

Savazzi, S.

Saxon, M. A.

Sbordone, F. L.

Schiltz, C.
SYMPOSIUM 1: 118

TALKS: 116

SYMPOSIUM 11: General abstract, 185; SYMPOSIUM 12: 150

TALKS: 210

POSTERS: 133

SYMPOSIUM 10: General abstract, 130, 148

POSTERS: 63

TALKS: 142

TALKS: 116

SYMPOSIUM 2: 176, TALKS: 145

TALKS: 62

POSTERS: 133
Pazzaglia, F. $\quad$ SYMPOSIUM 2: General abstract, 32

Pepperell, R. TALKS: 154

Pérennou, D. TALKS: 107, 110; POSTERS: 108

Petrarca, M. POSTERS: 195

Peviani, V. TALKS: 14

Piccardi, L. POSTERS: 41

Pietras, K. TALKS: 154

Piller, S.

SYMPOSIUM 13: 49

Piscicelli, C.

Polikarpus, S.

TALKS: 107, 110; POSTERS: 108

Ponticorvo, M.

Pöppel, E.

Prabhakar, A.

Presti, P.

Protzko, J.

TALKS: 126

Quesnot, T.

Quinton, J.

Rahe, M. SYMPOSIUM 9: 201; POSTERS: 34, 186 SYMPOSIUM 8: General abstract, 137

TALKS: 51

SYMPOSIUM 6: 18

SYMPOSIUM 2: 101

SYMPOSIUM 1: General abstract, 179

TALKS: 61

Ranzini, M. TALKS: 172

Rapuano, M. POSTERS: 77, 79

Rasse, T. TALKS: 132

Rea, M.

SYMPOSIUM: 140

Riecke, B. E.

TALKS: 100, 151, 174

Riggsby, A.

TALKS: 16

Rinaldi, L.

Risoli, A.

SYMPOSIUM 10: 40

Robinson, S.

TALKS: 33

Roduta Roberts, M.

Romeo, Z. SYMPOSIUM 6: 19

Rossi, D.

TALKS: 177

Rossi, M.

POSTERS: 193

Ruggiero, G.

TALKS: 33

SYMPOSIUM 12: General abstract, 65; TALKS:

47, 62; POSTERS: 50, 77, 79

Ruotolo, F.

Ruta, N. TALKS: 62, POSTERS: 77, 79

Ruzzon, D.

TALKS: 154

Saccani, M. S.

SYMPOSIUM 6: 18

Salemme, R.

Sallis, G.

Salsano, I.

TALKS: 177

Salvaggio, S.

Sandini, G.

SYMPOSIUM 11: 156; TALKS: 210

Santangelo, V.
TALKS: 126

POSTERS: 169

SYMPOSIUM 7: 37

TALKS: 76

POSTERS: 169 
Schooler, J.

Schwering, A.

SYMPOSIUM 2: 101

SYMPOSIUM 14: 98

Sebastiani, R.

POSTERS: 4, 11

Semenza, C

TALKS: 172

Senna, I.

SYMPOSIUM 13: 49

Sensibaugh, T.

TALKS: 70

Servajean, P.

POSTERS: 67

Sestieri, C.

TALKS: 204

Shaeken, W.

TALKS: 15

Shi, S.

SYMPOSIUM 8: 134

Shtyrov, Y.

TALKS: 10

Signorini, S.

SYMPOSIUM 13: 31; TALKS: 173

Silcock, G.

TALKS: 126

Simione, L.

SYMPOSIUM 9: General abstract, 189, 192

Šķilters, J.

TALKS: 163; POSTERS: 157

Skov-Petersen, H. TALKS: 72

Smeding, A. TALKS: 61

Smith, B. KEYNOTE 2

Somma, F. POSTERS: 34, 186

Sommer, N. R. POSTERS: 178

Soyer, C.

Spanò, B.

Stahn, A.

Stefanucci, J.

Suchan, J.

Summa, S.

Sun, J.

Szewczyk, M.

Szubielska, M.

Taghizadeh, B.

Takács, S.

Taukar, M.

Taverna, V.

Thoelen, F.

Tieri, G.

Timpf, S.

Tinella, L.

Todeschini, M.

Toffalini, E.

Tommasino, $\mathrm{P}$.

Tong, $\mathrm{Y}$.

Tonnaer, C.

Tononi, G.

Torromino, G.

Tosoni, A.

Truy, E.
TALKS: 89

POSTERS: 169

KEYNOTE 6

SYMPOSIUM 2: 176; TALKS: 96, 145

POSTERS: 209

POSTERS: 195

TALKS: 183

TALKS: 68; POSTERS: 202

TALKS: 68

SYMPOSIUM 11: 175

POSTERS: 56

TALKS: 126

TALKS: 33

TALKS: 126

SYMPOSIUM 3: 208

SYMPOSIUM 14: General abstract, 43

TALKS: 106

TALKS: 210

SYMPOSIUM 2: 32

SYMPOSIUM 3: 207

TALKS: 117; POSTERS: 129

TALKS: 126

TALKS: 14

POSTERS: 193

SYMPOSIUM 3: 205; TALKS: 204

TALKS: 210
Tüchler, A. F. TALKS: 163

Tversky, B. TALKS: 214

Vadalà, R. $\quad$ POSTERS: 169

Valori, I. SYMPOSIUM 13: 46

Valzolgher, C. TALKS: 210

van der Ham, I. SYMPOSIUM 2: 28

van Dijck, J. SYMPOSIUM 7: General abstract, 39

Van Velzen, J. SYMPOSIUM 13: 120

Vasco, G. POSTERS: 195

Vastmans, J. TALKS: 126

Vecchiato, G. SYMPOSIUM 6: General abstract, 18

Verdelet, G. TALKS: 210

Verdier, A. TALKS: 110 ; POSTERS: 108

Vidal Badía, M. SYMPOSIUM 5: 48

Vitali, H. TALKS: 173

Volpari, E. SYMPOSIUM 10: 140

von der Heyde, M. TALKS: 100, 151, 174

von Stülpnagel, R. SYMPOSIUM 14: 42

Walker, S. TALKS: 126

Wang, T. TALKS: 114

Wang, X. SYMPOSIUM 8: 134

Wang, Y. TALKS: 149

Wang, Z. POSTERS: 131

Wang, S. TALKS: 123

Weewer, R. TALKS: 126

Witt, A. TALKS: 128

Xi, T. $\quad$ SYMPOSIUM 8: 119

Xu, Y. TALKS: 123; POSTERS: 63

Yang, H. TALKS: 84

Yin, $\mathrm{X}$ TALKS: 144

Yu, X. SYMPOSIUM 8: 137

Zanchi, S. $\quad$ TALKS: 76

Zanini, A. SYMPOSIUM 11: 156

Zarina, L. TALKS: 163; POSTERS:157

Zavagnin, M. TALKS: 44

Zeng, Y. SYMPOSIUM 8: 112

Zhang, Z. SYMPOSIUM 8: 102

Zhang, D. TALKS: 149

Zhang, J. TALKS: 153

Zhao, C. TALKS: 123

Zhao, Y. TALKS: 96

Zhou, Y. TALKS: 113

Zielasko, D. TALKS: 151

Zorzi, M. TALKS: $5,172,177$

Zucchelli, M. M. POSTERS: 41

Publisher's Note Springer Nature remains neutral with regard to jurisdictional claims in published maps and institutional affiliations. 